\title{
Quiver Varieties with Multiplicities, Weyl Groups of Non-Symmetric Kac-Moody Algebras, and Painlevé Equations
}

Daisuke YAMAKAWA †‡

$\dagger$ Centre de mathématiques Laurent Schwartz, École polytechnique, CNRS UMR 7640, ANR SÉDIGA, 91128 Palaiseau Cedex, France

$\ddagger$ Department of Mathematics, Graduate School of Science, Kobe University, Rokko, Kobe 657-8501, Japan

E-mail: yamakawa@math.kobe-u.ac.jp

Received March 19, 2010, in final form October 18, 2010; Published online October 26, 2010 doi:10.3842/SIGMA.2010.087

\begin{abstract}
To a finite quiver equipped with a positive integer on each of its vertices, we associate a holomorphic symplectic manifold having some parameters. This coincides with Nakajima's quiver variety with no stability parameter/framing if the integers attached on the vertices are all equal to one. The construction of reflection functors for quiver varieties are generalized to our case, in which these relate to simple reflections in the Weyl group of some symmetrizable, possibly non-symmetric Kac-Moody algebra. The moduli spaces of meromorphic connections on the rank 2 trivial bundle over the Riemann sphere are described as our manifolds. In our picture, the list of Dynkin diagrams for Painlevé equations is slightly different from (but equivalent to) Okamoto's.
\end{abstract}

Key words: quiver variety; quiver variety with multiplicities; non-symmetric Kac-Moody algebra; Painlevé equation; meromorphic connection; reflection functor; middle convolution

2010 Mathematics Subject Classification: 53D30; 16G20; 20F55; 34M55

\section{Introduction}

First, we briefly explain our main objects in this article. Let

- $\mathrm{Q}$ be a quiver, i.e., a directed graph, with the set of vertices $I$ (our quivers are always assumed to be finite and have no arrows joining a vertex with itself);

- $\mathbf{d}=\left(d_{i}\right)_{i \in I} \in \mathbb{Z}_{>0}^{I}$ be a collection of positive integers indexed by the vertices.

We think of each number $d_{i}$ as the 'multiplicity' of the vertex $i \in I$, so the pair $(\mathrm{Q}, \mathbf{d})$ as a 'quiver with multiplicities'. In this article, we associate to such $(\mathbf{Q}, \mathbf{d})$ a holomorphic symplectic manifold $\mathcal{N}_{\mathbf{Q}, \mathbf{d}}^{\mathrm{s}}(\lambda, \mathbf{v})$ having parameters

- $\lambda=\left(\lambda_{i}(z)\right)_{i \in I}$, where $\lambda_{i}(z)=\lambda_{i, 1} z^{-1}+\lambda_{i, 2} z^{-2}+\cdots+\lambda_{i, d_{i}} z^{-d_{i}} \in z^{-d_{i}} \mathbb{C}[z] / \mathbb{C}[z]$

- $\mathbf{v}=\left(v_{i}\right)_{i \in I} \in \mathbb{Z}_{\geq 0}^{I}$,

and call it the quiver variety with multiplicities, because if $d_{i}=1$ for all $i \in I$, it then coincides with (the stable locus of) Nakajima's quiver variety $\mathfrak{M}_{\zeta}^{\text {reg }}(\mathbf{v}, \mathbf{w})[21]$ with

$$
\mathbf{w}=0 \in \mathbb{Z}_{\geq 0}^{I}, \quad \zeta=\left(\zeta_{\mathbb{R}}, \zeta_{\mathbb{C}}\right)=\left(0,\left(\lambda_{i, 1}\right)_{i \in I}\right) \in \sqrt{-1} \mathbb{R}^{I} \times \mathbb{C}^{I} .
$$

As in the case of quiver variety, $\mathcal{N}_{\mathbf{Q}, \mathbf{d}}^{\mathrm{s}}(\lambda, \mathbf{v})$ is defined as a holomorphic symplectic quotient with respect to some algebraic group action (see Section 3). However, the group used here is 
non-reductive unless $d_{i}=1$ or $v_{i}=0$ for all $i \in I$. Therefore a number of basic facts in the theory of holomorphic symplectic quotients (e.g. the hyper-Kähler quotient description) cannot be applied to our $\mathcal{N}_{\mathbf{Q}, \mathbf{d}}^{\mathrm{s}}(\lambda, \mathbf{v})$, and for the same reason, they seem to provide new geometric objects relating to quivers.

The definition of $\mathcal{N}_{Q, \mathbf{d}}^{\mathrm{s}}(\lambda, \mathbf{v})$ is motivated by the theory of Painlevé equations. It is known due to Okamoto's work $[23,24,25,26]$ that all Painlevé equations except the first one have (extended) affine Weyl group symmetries; see the table below, where $P_{J}$ denotes the Painlevé equation of type $J(J=$ II, III, ...,VI).

\begin{tabular}{|c||l|l|l|l|l|}
\hline Equations & $P_{\mathrm{VI}}$ & $P_{\mathrm{V}}$ & $P_{\mathrm{IV}}$ & $P_{\mathrm{III}}$ & $P_{\mathrm{II}}$ \\
\hline Symmetries & $D_{4}^{(1)}$ & $A_{3}^{(1)}$ & $A_{2}^{(1)}$ & $C_{2}^{(1)}$ & $A_{1}^{(1)}$ \\
\hline
\end{tabular}

On the other hand, each of them is known to govern an isomonodromic deformation of rank two meromorphic connections on $\mathbb{P}^{1}[12]$; the number of poles and the pole orders of connections remain unchanged during the deformation, and are determined from (if we assume that the connections have only 'unramified' singularities) the type of the Painlevé equation (see e.g. [27]). See the table below, where $k_{1}+k_{2}+\cdots+k_{n}$ means that the connections in the deformation have $n$ poles of order $k_{i}, i=1,2, \ldots, n$ and no other poles.

\begin{tabular}{|c||c|c|c|c|c|}
\hline Equations & $P_{\mathrm{VI}}$ & $P_{\mathrm{V}}$ & $P_{\mathrm{IV}}$ & $P_{\mathrm{III}}$ & $P_{\mathrm{II}}$ \\
\hline Connections & $1+1+1+1$ & $2+1+1$ & $3+1$ & $2+2$ & 4 \\
\hline
\end{tabular}

Roughly speaking, we thus have a non-trivial correspondence between some Dynkin diagrams and rank two meromorphic connections.

In fact, such a relationship can be understood in terms of quiver varieties except in the case of $P_{\text {III }}$. Crawley-Boevey [7] described the moduli spaces of Fuchsian systems (i.e., meromorphic connections on the trivial bundle over $\mathbb{P}^{1}$ having only simple poles) as quiver varieties associated with 'star-shaped' quivers. In particular, the moduli space of rank two Fuchsian systems having exactly four poles are described as a quiver variety of type $D_{4}^{(1)}$, which is consistent with the above correspondence for $P_{\mathrm{VI}}$. The quiver description in the cases of $P_{\mathrm{II}}, P_{\mathrm{IV}}$ and $P_{\mathrm{V}}$ was obtained by Boalch ${ }^{1}$ [4]; more generally, he proved that the moduli spaces of meromorphic connections on the trivial bundle over $\mathbb{P}^{1}$ having one higher order pole (and possibly simple poles) are quiver varieties.

A remarkable point is that their quiver description provides Weyl group symmetries of the moduli spaces ${ }^{2}$ at the same time, because for any quiver, the associated quiver varieties are known to have such symmetry. This is generated by the so-called reflection functors (see Theorem 1.2 below), whose existence was first announced by Nakajima (see [21, Section 9], where he also gave its geometric proof in some important cases), and then shown by several researchers including himself $[8,19,22,28]$.

The purpose of quiver varieties with multiplicities is to generalize their description to the case of $P_{\mathrm{III}}$; the starting point is the following observation (see Proposition 6.6 for a further generalized, precise statement; see also Remarks 6.4 and 6.5):

Proposition 1.1. Consider a 'star-shaped quiver of length one'

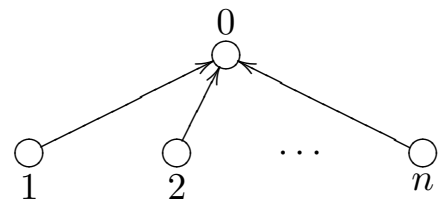

\footnotetext{
${ }^{1}$ His description in the case of $P_{\mathrm{V}}$ is based on the work of Harnad [10].

${ }^{2}$ Actually in each Painlevé case, this action reduces to an action of the corresponding finite Weyl group, which together with 'Schlesinger transformations' give the full symmetry; see [5, Section 6].
} 
Here the set of vertices is $I=\{0,1, \ldots, n\}$. Take multiplicities $\mathbf{d} \in \mathbb{Z}_{>0}^{I}$ with $d_{0}=1$ and set $\mathbf{v} \in \mathbb{Z}_{\geq 0}^{I}$ by $v_{0}=2, v_{i}=1(i=1, \ldots, n)$. Then $\mathcal{N}_{\mathbf{Q}, \mathbf{d}}^{\mathrm{s}}(\lambda, \mathbf{v})$ is isomorphic to the moduli space of stable meromorphic connections on the rank two trivial bundle over $\mathbb{P}^{1}$ having $n$ poles of order $d_{i}$, $i=1, \ldots, n$ of prescribed formal type.

On the other hand, to any quiver with multiplicities, we associate a generalized Cartan matrix $\mathbf{C}$ as follows:

$$
\mathbf{C}=2 \mathrm{Id}-\mathbf{A D},
$$

where $\mathbf{A}$ is the adjacency matrix of the underlying graph, namely, the matrix whose $(i, j)$ entry is the number of edges joining $i$ and $j$, and $\mathbf{D}$ is the diagonal matrix with entries given by the multiplicities $\mathbf{d}$. It is symmetrizable as $\mathbf{D C}$ is symmetric, but may be not symmetric.

Now as stated below, our quiver varieties with multiplicities admit reflection functors; this is the main result of this article.

Theorem 1.2 (see Section 4). For any quiver with multiplicities (Q,d), there exist linear maps

$$
s_{i}: \mathbb{Z}^{I} \rightarrow \mathbb{Z}^{I}, \quad r_{i}: \bigoplus_{i \in I}\left(z^{-d_{i}} \mathbb{C}[z] / \mathbb{C}[z]\right) \rightarrow \bigoplus_{i \in I}\left(z^{-d_{i}} \mathbb{C}[z] / \mathbb{C}[z]\right) \quad(i \in I)
$$

generating actions of the Weyl group of the associated Kac-Moody algebra, such that for any $(\lambda, \mathbf{v})$ and $i \in I$ with $\lambda_{i, d_{i}} \neq 0$, one has a natural symplectomorphism

$$
\mathcal{F}_{i}: \quad \mathcal{N}_{\mathrm{Q}, \mathbf{d}}^{\mathrm{s}}(\lambda, \mathbf{v}) \stackrel{\simeq}{\longrightarrow} \mathcal{N}_{\mathrm{Q}, \mathbf{d}}^{\mathrm{s}}\left(r_{i}(\lambda), s_{i}(\mathbf{v})\right) .
$$

If $d_{i}=1$ for all $i \in I$, then the maps $\mathcal{F}_{i}$ coincide with the reflection functors.

In the case of star-shaped quivers, the original reflection functor at the central vertex can be interpreted in terms of Katz's middle convolution [14] for Fuchsian systems (see [3, Appendix A]). A similar assertion also holds in the situation of Proposition 1.1; the map $\mathcal{F}_{0}$ at the central vertex 0 can be interpreted in terms of the 'generalized middle convolution' [1, 31] (see Section 6.3).

For instance, consider the star-shaped quivers with multiplicities given below
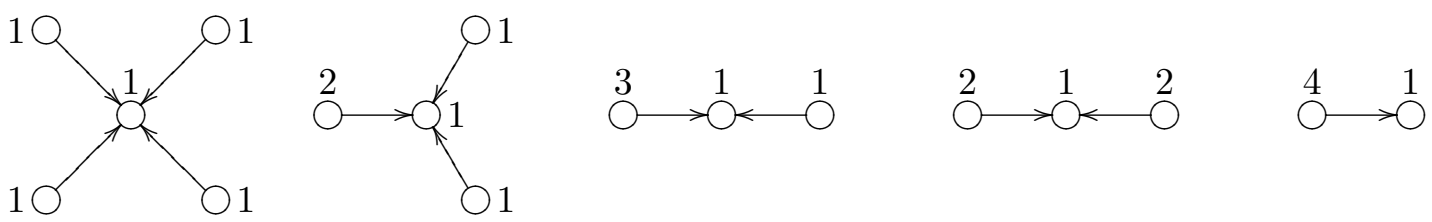

Proposition 1.1 says that the associated $\mathcal{N}_{\mathbf{Q}, \mathbf{d}}^{\mathrm{s}}(\lambda, \mathbf{v})$ with a particular choice of $\mathbf{v}$ give the moduli spaces for $P_{\mathrm{VI}}, P_{\mathrm{V}}, P_{\mathrm{IV}}, P_{\mathrm{III}}$ and $P_{\mathrm{II}}$, respectively. On the other hand, the associated Kac-Moody algebras are respectively given $b^{3}$

$$
D_{4}^{(1)}, \quad A_{5}^{(2)}, \quad D_{4}^{(3)}, \quad C_{2}^{(1)}, \quad A_{2}^{(2)} .
$$

Interestingly, this list of Kac-Moody algebras is different from the table given before; however we can clarify the relationship between our description and Boalch's by using a sort of 'shifting trick' established by him (see Section 5.1). This trick, which may be viewed as a geometric phenomenon arising from the "normalization of the leading coefficient in the principal part of the connection at an irregular singular point', connects two quiver varieties with multiplicities associated to different quivers with multiplicities; more specifically, we prove the following:

\footnotetext{
${ }^{3}$ We follow Kac [13] for the notation of (twisted) affine Lie algebras.
} 
Theorem 1.3 (see Section 5). Suppose that a quiver with multiplicities (Q,d) has a pair of vertices $(i, j)$ such that

$$
d_{i}>1, \quad d_{j}=1, \quad a_{i k}=a_{k i}=\delta_{j k} \quad \text { for any } k \in I,
$$

where $\mathbf{A}=\left(a_{i j}\right)$ is the adjacency matrix of the underlying graph. Then it determines another quiver with multiplicities $(\check{\mathbf{Q}}, \check{\mathbf{d}})$ and a map $(\lambda, \mathbf{v}) \mapsto(\check{\lambda}, \check{\mathbf{v}})$ between parameters such that the following holds: if $\lambda_{i, d_{i}} \neq 0$, then $\mathcal{N}_{\mathbf{Q}, \mathbf{d}}^{\mathrm{s}}(\lambda, \mathbf{v})$ and $\mathcal{N}_{\check{Q}, \check{\mathbf{d}}}^{\mathrm{s}}(\check{\lambda}, \check{\mathbf{v}})$ are symplectomorphic to each other.

We call the transformation $(\mathbf{Q}, \mathbf{d}) \mapsto(\check{Q}, \check{\mathbf{d}})$, whose precise definition is given in Section 5.2 , the normalization. Using this theorem, we can translate the above list of Dynkin diagrams into the original one (see Section 6.4).

There is a close relationship between two Kac-Moody root systems connected via the normalization (see Section 5.3). In particular, we have the following relation between the Weyl groups $W, \check{W}$ associated to $(\mathbf{Q}, \mathbf{d}),(\check{Q}, \check{\mathbf{d}})$ :

$$
W \simeq \check{W} \rtimes \mathbb{Z} / 2 \mathbb{Z},
$$

where the semidirect product is taken with respect to some Dynkin automorphism of order 2 (such a Dynkin automorphism canonically exists by the definition of normalization). For instance, in the cases of $P_{\mathrm{V}}, P_{\mathrm{IV}}$ and $P_{\mathrm{II}}$, we have

$$
\begin{aligned}
& W\left(A_{5}^{(2)}\right) \simeq W\left(A_{3}^{(1)}\right) \rtimes \mathbb{Z} / 2 \mathbb{Z}, \\
& W\left(D_{4}^{(3)}\right) \simeq W\left(A_{2}^{(1)}\right) \rtimes \mathbb{Z} / 2 \mathbb{Z}, \\
& W\left(A_{2}^{(2)}\right) \simeq W\left(A_{1}^{(1)}\right) \rtimes \mathbb{Z} / 2 \mathbb{Z},
\end{aligned}
$$

which mean that our list of Dynkin diagrams for Painlevé equations is a variant of Okamoto's obtained by (partially) extending the Weyl groups.

\section{Preliminaries}

In this section we briefly recall the definition of Nakajima's quiver variety [21].

\subsection{Quiver}

Recall that a (finite) quiver is a quadruple $\mathrm{Q}=(I, \Omega, \mathrm{s}, \mathrm{t}$ ) consisting of two finite sets $I, \Omega$ (the set of vertices, resp. arrows) and two maps s, t: $\Omega \rightarrow I$ (assigning to each arrow its source, resp. target). Throughout this article, for simplicity, we assume that our quivers $\mathrm{Q}$ have no arrow $h \in \Omega$ with $\mathrm{s}(h)=\mathrm{t}(h)$.

For given $\mathrm{Q}$, we denote by $\overline{\mathrm{Q}}=(I, \bar{\Omega}, \mathrm{s}, \mathrm{t})$ the quiver obtained from $\mathrm{Q}$ by reversing the orientation of each arrow; the set $\bar{\Omega}=\{\bar{h} \mid h \in \Omega\}$ is just a copy of $\Omega$, and $\mathrm{s}(\bar{h}):=\mathrm{t}(h)$, $\mathrm{t}(\bar{h}):=\mathrm{s}(h)$ for $h \in \Omega$. We set $H:=\Omega \sqcup \bar{\Omega}$, and extend the map $\Omega \rightarrow \bar{\Omega}, h \mapsto \bar{h}$ to an involution of $H$ in the obvious way. The resulting quiver $\mathrm{Q}+\overline{\mathrm{Q}}=(I, H, \mathrm{~s}, \mathrm{t})$ is called the double of $\mathrm{Q}$.

The underlying graph of $\mathrm{Q}$, which is obtained by forgetting the orientation of each arrow, determines a symmetric matrix $\mathbf{A}=\left(a_{i j}\right)_{i, j \in I}$, called the adjacency matrix, as follows:

$$
a_{i j}:=\sharp\{\text { edges joining } i \text { and } j\}=\sharp\{h \in H \mid \mathrm{s}(h)=i, \mathrm{t}(h)=j\} .
$$

Let $\mathbf{V}=\bigoplus_{i \in I} V_{i}$ be a nonzero finite-dimensional $I$-graded $\mathbb{C}$-vector space. A representation of $\mathbf{Q}$ over $\mathbf{V}$ is an element of the vector space

$$
\operatorname{Rep}_{\mathbf{Q}}(\mathbf{V}):=\bigoplus_{h \in \Omega} \operatorname{Hom}_{\mathbb{C}}\left(V_{\mathbf{s}(h)}, V_{\mathbf{t}(h)}\right),
$$


and its dimension vector is given by $\mathbf{v}:=\operatorname{dim} \mathbf{V} \equiv\left(\operatorname{dim} V_{i}\right)_{i \in I}$. Isomorphism classes of representations of $\mathbf{Q}$ with dimension vector $\mathbf{v}$ just correspond to orbits in $\operatorname{Rep}_{\mathbf{Q}}(\mathbf{V})$ with respect to the action of the group $\mathrm{GL}(\mathbf{V}):=\prod_{i \in I} \mathrm{GL}_{\mathbb{C}}\left(V_{i}\right)$ given by

$$
g=\left(g_{i}\right):\left(B_{h}\right)_{h \in \Omega} \longmapsto\left(g_{\mathrm{t}(h)} B_{h} g_{\mathbf{s}(h)}^{-1}\right)_{h \in \Omega}, \quad g \in \mathrm{GL}(\mathbf{V}) .
$$

We denote the Lie algebra of $\mathrm{GL}(\mathbf{V})$ by $\mathfrak{g l}(\mathbf{V})$; explicitly, $\mathfrak{g l}(\mathbf{V}):=\bigoplus_{i \in I} \mathfrak{g l}_{\mathbb{C}}\left(V_{i}\right)$. For $\zeta=$ $\left(\zeta_{i}\right)_{i \in I} \in \mathbb{C}^{I}$, we denote its image under the natural map $\mathbb{C}^{I} \rightarrow \mathfrak{g l}(\mathbf{V})$ by $\zeta \operatorname{Id}_{\mathbf{V}}$, and also use the same letter $\zeta \operatorname{Id}_{\mathbf{V}}$ for $\zeta \in \mathbb{C}$ via the diagonal embedding $\mathbb{C} \hookrightarrow \mathbb{C}^{I}$. Note that the central subgroup $\mathbb{C}^{\times} \simeq\left\{\zeta \operatorname{Id}_{\mathbf{V}} \mid \zeta \in \mathbb{C}^{\times}\right\} \subset \mathrm{GL}(\mathbf{V})$ acts trivially on $\operatorname{Rep}_{\mathbf{Q}}(\mathbf{V})$, so we have the induced action of the quotient group $\mathrm{GL}(\mathbf{V}) / \mathbb{C}^{\times}$.

Let $B=\left(B_{h}\right)_{h \in \Omega} \in \operatorname{Rep}_{\mathbf{Q}}(\mathbf{V})$. An $I$-graded subspace $\mathbf{S}=\bigoplus_{i \in I} S_{i}$ of $\mathbf{V}$ is said to be $B$ invariant if $B_{h}\left(S_{\mathbf{s}(h)}\right) \subset S_{\mathrm{t}(h)}$ for all $h \in \Omega$. If $\mathbf{V}$ has no $B$-invariant subspace except $\mathbf{S}=0, \mathbf{V}$, then $B$ is said to be irreducible. Schur's lemma ${ }^{4}$ implies that the stabilizer of each irreducible $B$ is just the central subgroup $\mathbb{C}^{\times} \subset \mathrm{GL}(\mathbf{V})$, and a standard fact in Mumford's geometric invariant theory [20, Corollary 2.5] (see also [16]) implies that the action of $\mathrm{GL}(\mathbf{V}) / \mathbb{C}^{\times}$on the subset $\operatorname{Rep}_{Q}^{\mathrm{irr}}(\mathbf{V})$ consisting of all irreducible representations over $\mathbf{V}$ is proper.

\subsection{Quiver variety}

Suppose that a quiver $\mathrm{Q}$ and a nonzero finite-dimensional $I$-graded $\mathbb{C}$-vector space $\mathbf{V}=\bigoplus_{i \in I} V_{i}$ are given. We set

$$
\mathbf{M}_{\mathbf{Q}}(\mathbf{V}):=\operatorname{Rep}_{\mathbf{Q}+\overline{\mathbf{Q}}}(\mathbf{V})=\operatorname{Rep}_{\mathbf{Q}}(\mathbf{V}) \oplus \operatorname{Rep}_{\bar{Q}}(\mathbf{V}),
$$

and regard it as the cotangent bundle of $\operatorname{Rep}_{\mathbf{Q}}(\mathbf{V})$ by using the trace pairing. Introducing the function

$$
\epsilon: H \rightarrow\{ \pm 1\}, \quad \epsilon(h):=\left\{\begin{aligned}
1 & \text { for } h \in \Omega \\
-1 & \text { for } h \in \bar{\Omega}
\end{aligned}\right.
$$

we can write the canonical symplectic form on $\mathbf{M}_{\mathbf{Q}}(\mathbf{V})$ as

$$
\omega:=\sum_{h \in \Omega} \operatorname{tr} \mathrm{d} B_{h} \wedge \mathrm{d} B_{\bar{h}}=\frac{1}{2} \sum_{h \in H} \epsilon(h) \operatorname{tr} \mathrm{d} B_{h} \wedge \mathrm{d} B_{\bar{h}}, \quad\left(B_{h}\right)_{h \in H} \in \mathbf{M}_{\mathbf{Q}}(\mathbf{V}) .
$$

The natural $\mathrm{GL}(\mathbf{V})$-action on $\mathbf{M}_{\mathbf{Q}}(\mathbf{V})$ is Hamiltonian with respect to $\omega$ with the moment map

$$
\mu=\left(\mu_{i}\right)_{i \in I}: \mathbf{M}_{\mathbf{Q}}(\mathbf{V}) \rightarrow \mathfrak{g l}(\mathbf{V}), \quad \mu_{i}(B)=\sum_{\substack{h \in H: \\ \mathrm{t}(h)=i}} \epsilon(h) B_{h} B_{\bar{h}}
$$

vanishing at the origin, where we identify $\mathfrak{g l}(\mathbf{V})$ with its dual using the trace pairing.

Definition 2.1. A point $B \in \mathbf{M}_{\mathbf{Q}}(\mathbf{V})$ is said to be stable if it is irreducible as a representation of $\mathrm{Q}+\overline{\mathrm{Q}}$.

For a $\mathrm{GL}(\mathbf{V})$-invariant Zariski closed subset $Z$ of $\mathbf{M}_{\mathbf{Q}}(\mathbf{V})$, let $Z^{\text {s }}$ be the subset of all stable points in $Z$. It is a $\mathrm{GL}(\mathbf{V})$-invariant Zariski open subset of $Z$, on which the group $\mathrm{GL}(\mathbf{V}) / \mathbb{C}^{\times}$ acts freely and properly.

\footnotetext{
${ }^{4}$ One can apply Schur's lemma thanks to the following well-known fact: the category of representations of Q is equivalent to that of an algebra $\mathbb{C Q}$, the so-called path algebra; see e.g. [9].
} 
Definition 2.2. For $\zeta \in \mathbb{C}^{I}$ and $\mathbf{v} \in \mathbb{Z}_{\geq 0}^{I} \backslash\{0\}$, taking an $I$-graded $\mathbb{C}$-vector space $\mathbf{V}$ with $\operatorname{dim} \mathbf{V}=\mathbf{v}$ we define

$$
\mathcal{N}_{\mathbf{Q}}^{\mathrm{s}}(\zeta, \mathbf{v}):=\mu^{-1}\left(-\zeta \operatorname{Id}_{\mathbf{V}}\right)^{\mathrm{s}} / \mathrm{GL}(\mathbf{V}),
$$

which we call the quiver variety.

Remark 2.3. In Nakajima's notation (see [21] or $[22]), \mathcal{N}_{\mathbf{Q}}^{\mathbf{s}}(\zeta, \mathbf{v})$ is denoted by $\mathfrak{M}_{(0, \zeta)}^{\mathrm{reg}}(\mathbf{v}, 0)$.

\section{Quiver variety with multiplicities}

\subsection{Definition}

For a positive integer $d$, we set

$$
R_{d}:=\mathbb{C}[[z]] / z^{d} \mathbb{C}[[z]], \quad R^{d}:=z^{-d} \mathbb{C}[[z]] / \mathbb{C}[[z]] .
$$

The $\mathbb{C}$-algebra $R_{d}$ has a typical basis $\left\{z^{d-1}, \ldots, z, 1\right\}$, with respect to which the multiplication by $z$ in $R_{d}$ is represented by the nilpotent single Jordan block

$$
J_{d}:=\left(\begin{array}{cccc}
0 & 1 & & 0 \\
& 0 & \ddots & \\
& & \ddots & 1 \\
0 & & & 0
\end{array}\right) \in \operatorname{End}\left(\mathbb{C}^{d}\right)=\operatorname{End}_{\mathbb{C}}\left(R_{d}\right) .
$$

The vector space $R^{d}$ may be identified with the $\mathbb{C}$-dual $R_{d}^{*}=\operatorname{Hom}_{\mathbb{C}}\left(R_{d}, \mathbb{C}\right)$ of $R_{d}$ via the pairing

$$
R_{d} \otimes_{\mathbb{C}} R^{d} \rightarrow \mathbb{C}, \quad(f, g) \mapsto \operatorname{res}_{z=0}(f(z) g(z)) .
$$

For a finite-dimensional $\mathbb{C}$-vector space $V$, we set

$$
\mathfrak{g}_{d}(V):=\mathfrak{g l}(V) \otimes_{\mathbb{C}} R_{d}=\mathfrak{g l}(V)[[z]] / z^{d} \mathfrak{g l}(V)[[z]] .
$$

Note that $\mathfrak{g}_{d}(V)$ is naturally isomorphic to $\operatorname{End}_{R_{d}}\left(V \otimes_{\mathbb{C}} R_{d}\right)$ as an $R_{d}$-module; hence it is the Lie algebra of the complex algebraic group

$$
G_{d}(V):=\operatorname{Aut}_{R_{d}}\left(V \otimes_{\mathbb{C}} R_{d}\right) \simeq\left\{g(z)=\sum_{k=0}^{d-1} g_{k} z^{k} \in \mathfrak{g}_{d}(V) \mid \operatorname{det} g_{0} \neq 0\right\} .
$$

The inverse element of $g(z) \in G_{d}(V)$ is given by taking modulo $z^{d} \mathfrak{g r}(V)[[z]]$ of the formal inverse $g(z)^{-1} \in \mathfrak{g l}(V)[[z]]$. The adjoint action of $g(z)$ is described as

$$
(g \cdot \xi)(z)=g(z) \xi(z) g(z)^{-1} \quad \bmod z^{d} \mathfrak{g l}(V)[[z]], \quad \xi(z) \in \mathfrak{g}_{d}(V) .
$$

Using the above $R_{d}^{*} \simeq R^{d}$ and the trace pairing, we always identify the $\mathbb{C}$-dual $\mathfrak{g}_{d}^{*}(V)$ of $\mathfrak{g}_{d}(V)$ with $\mathfrak{g l}(V) \otimes_{\mathbb{C}} R^{d}=z^{-d} \mathfrak{g l}(V)[[z]] / \mathfrak{g l}(V)[[z]]$. Then the coadjoint action of $g(z) \in G_{d}(V)$ is also described as

$$
(g \cdot \eta)(z)=g(z) \eta(z) g(z)^{-1} \quad \bmod \mathfrak{g l}(V)[[z]], \quad \eta(z)=\sum_{k=1}^{d} \eta_{k} z^{-k} \in \mathfrak{g}_{d}^{*}(V) .
$$

The natural inclusion $\mathfrak{g}_{d}(V) \hookrightarrow \operatorname{End}_{\mathbb{C}}\left(V \otimes_{\mathbb{C}} R_{d}\right)=\operatorname{End}_{\mathbb{C}}(V) \otimes_{\mathbb{C}} \operatorname{End}_{\mathbb{C}}\left(R_{d}\right)$ is represented by

$$
\xi(z)=\sum_{k=0}^{d-1} \xi_{k} z^{k} \longmapsto \sum_{k=0}^{d-1} \xi_{k} \otimes J_{d}^{k}
$$


whose image is just the centralizer of $\operatorname{Id}_{V} \otimes J_{d}$. Accordingly, its transpose can be written as

$$
\mathfrak{g l}_{\mathbb{C}}\left(V \otimes_{\mathbb{C}} R_{d}\right) \simeq \mathfrak{g l}_{\mathbb{C}}\left(V \otimes_{\mathbb{C}} R_{d}\right)^{*} \rightarrow \mathfrak{g}_{d}^{*}(V), \quad X \mapsto \sum_{k=1}^{d} \operatorname{tr}_{R_{d}}\left[X\left(\operatorname{Id}_{V} \otimes J_{d}^{k-1}\right)\right] z^{-k}
$$

where $\operatorname{tr}_{R_{d}}: \operatorname{End}_{\mathbb{C}}\left(V \otimes_{\mathbb{C}} R_{d}\right)=\operatorname{End}_{\mathbb{C}}(V) \otimes_{\mathbb{C}} \operatorname{End}_{\mathbb{C}}\left(R_{d}\right) \rightarrow \operatorname{End}_{\mathbb{C}}(V)$ denotes the trace of the $\operatorname{End}_{\mathbb{C}}\left(R_{d}\right)$-part.

Now suppose that a quiver $\mathbf{Q}$ and a collection of positive integers $\mathbf{d}=\left(d_{i}\right)_{i \in I} \in \mathbb{Z}_{>0}^{I}$ are given. We call the pair $(\mathrm{Q}, \mathbf{d})$ as a quiver with multiplicities and $d_{i}$ as the multiplicity of the vertex $i$. Set

$$
R_{\mathbf{d}}:=\bigoplus_{i \in I} R_{d_{i}}, \quad R^{\mathbf{d}}:=\bigoplus_{i \in I} R^{d_{i}}
$$

and for a nonzero finite-dimensional $I$-graded $\mathbb{C}$-vector space $\mathbf{V}=\bigoplus_{i \in I} V_{i}$, set

$$
\begin{aligned}
& \mathbf{V}_{\mathbf{d}} \equiv \mathbf{V} \otimes_{\mathbb{C}} R_{\mathbf{d}}:=\bigoplus_{i \in I} V_{i} \otimes_{\mathbb{C}} R_{d_{i}}, \\
& \mathbf{M}_{\mathbf{Q}, \mathbf{d}}(\mathbf{V}):=\mathbf{M}_{\mathbf{Q}}\left(\mathbf{V}_{\mathbf{d}}\right)=\bigoplus_{h \in H} \operatorname{Hom}_{\mathbb{C}}\left(V_{\mathbf{s}(h)} \otimes_{\mathbb{C}} R_{d_{\mathbf{s}(h)}}, V_{\mathbf{t}(h)} \otimes_{\mathbb{C}} R_{d_{\mathbf{t}(h)}}\right), \\
& G_{\mathbf{d}}(\mathbf{V}):=\prod_{i \in I} G_{d_{i}}\left(V_{i}\right), \quad \mathfrak{g}_{\mathbf{d}}(\mathbf{V}):=\bigoplus_{i \in I} \mathfrak{g}_{d_{i}}\left(V_{i}\right) .
\end{aligned}
$$

The group $G_{\mathbf{d}}(\mathbf{V})$ naturally acts on $\mathbf{M}_{\mathbf{Q}, \mathbf{d}}(\mathbf{V})$ as a subgroup of $\mathrm{GL}\left(\mathbf{V}_{\mathbf{d}}\right)$. Note that the subgroup $\mathbb{C}^{\times} \subset \mathrm{GL}\left(\mathbf{V}_{\mathbf{d}}\right)$ is contained in $G_{\mathbf{d}}(\mathbf{V})$ and acts trivially on $\mathbf{M}_{\mathbf{Q}, \mathbf{d}}(\mathbf{V})$. As in the case of $\mathfrak{g l}(\mathbf{V})$, for $\lambda=\left(\lambda_{i}(z)\right)_{i \in I} \in R^{\mathbf{d}}$ we denote its image under the natural map $R^{\mathbf{d}}=\mathfrak{g}_{\mathbf{d}}^{*}\left(\mathbb{C}^{I}\right) \rightarrow \mathfrak{g}_{\mathbf{d}}^{*}(\mathbf{V})$ by $\lambda \operatorname{Id}_{\mathbf{V}}$.

Let $\omega$ be the canonical symplectic form on $\mathbf{M}_{Q, \mathbf{d}}(\mathbf{V})$;

$$
\omega=\frac{1}{2} \sum_{h \in H} \epsilon(h) \operatorname{tr} \mathrm{d} B_{h} \wedge \mathrm{d} B_{\bar{h}}, \quad\left(B_{h}\right)_{h \in H} \in \mathbf{M}_{\mathbf{Q}, \mathbf{d}}(\mathbf{V})
$$

Then the $G_{\mathbf{d}}(\mathbf{V})$-action is Hamiltonian whose moment map $\mu_{\mathbf{d}}$ is given by the composite of the $\mathrm{GL}\left(\mathbf{V}_{\mathbf{d}}\right)$-moment map $\mu=\left(\mu_{i}\right): \mathbf{M}_{\mathbf{Q}, \mathbf{d}}(\mathbf{V}) \rightarrow \mathfrak{g l}\left(\mathbf{V}_{\mathbf{d}}\right)$ (see (2.1) for the definition) and the transpose $\operatorname{pr}=\left(\operatorname{pr}_{i}\right)$ of the inclusion $\mathfrak{g}_{\mathbf{d}}(\mathbf{V}) \hookrightarrow \mathfrak{g l}\left(\mathbf{V}_{\mathbf{d}}\right)$;

$$
\begin{aligned}
& \mu_{\mathbf{d}}=\left(\mu_{\mathbf{d}, i}\right)_{i \in I}: \mathbf{M}_{\mathbf{Q}, \mathbf{d}}(\mathbf{V}) \rightarrow \mathfrak{g}_{\mathbf{d}}^{*}(\mathbf{V}), \\
& \mu_{\mathbf{d}, i}(B):=\operatorname{pr}_{i} \circ \mu_{i}(B)=\sum_{k=1}^{d_{i}} \sum_{\substack{h \in H: \\
\mathbf{t}(h)=i}} \epsilon(h) \operatorname{tr}_{R_{d_{i}}}\left[B_{h} B_{\bar{h}} N_{i}^{k-1}\right] z^{-k},
\end{aligned}
$$

where $N_{i}:=\operatorname{Id}_{V_{i}} \otimes J_{d_{i}}$.

Definition 3.1. A point $B \in \mathbf{M}_{\mathbf{Q}, \mathbf{d}}(\mathbf{V})$ is said to be stable if $\mathbf{V}_{\mathbf{d}}$ has no nonzero proper $B$ invariant subspace $\mathbf{S}=\bigoplus_{i \in I} S_{i}$ such that $S_{i} \subset V_{i} \otimes_{\mathbb{C}} R_{d_{i}}$ is an $R_{d_{i}}$-submodule for each $i \in I$.

The above stability can be interpreted in terms of the irreducibility of representations of a quiver. Letting $\widetilde{\Omega}:=\Omega \sqcup\left\{\ell_{i}\right\}_{i \in I}$ and extending the maps s, t to $\widetilde{\Omega}$ by $\mathrm{s}\left(\ell_{i}\right)=\mathrm{t}\left(\ell_{i}\right)=i$, we obtain a new quiver $\widetilde{\mathrm{Q}}=(I, \widetilde{\Omega}, \mathrm{s}, \mathrm{t})$. Consider the vector space

$$
\operatorname{Rep}_{\widetilde{Q}+\overline{\mathbf{Q}}}\left(\mathbf{V}_{\mathbf{d}}\right) \simeq \mathbf{M}_{\mathbf{Q}, \mathbf{d}}(\mathbf{V}) \oplus \mathfrak{g l}\left(\mathbf{V}_{\mathbf{d}}\right)
$$


associated with the quiver $\widetilde{\mathrm{Q}}+\overline{\mathrm{Q}}=(I, \widetilde{\Omega} \sqcup \bar{\Omega}, \mathrm{s}, \mathrm{t})$. Note that in the above definition, a vector subspace $S_{i} \subset V_{i} \otimes R_{d_{i}}$ is an $R_{d_{i}}$-submodule if and only if it is invariant under the action of $N_{i}=\operatorname{Id}_{V_{i}} \otimes J_{d_{i}}$, which corresponds to the multiplication by $z$. Thus letting

$$
\iota: \quad \mathbf{M}_{\mathbf{Q}, \mathbf{d}}(\mathbf{V}) \hookrightarrow \operatorname{Rep}_{\widetilde{\mathbf{Q}}+\overline{\mathbf{Q}}}\left(\mathbf{V}_{\mathbf{d}}\right), \quad B \mapsto\left(B,\left(N_{i}\right)_{i \in I}\right),
$$

we see that a point $B \in \mathbf{M}_{\mathbf{Q}, \mathbf{d}}(\mathbf{V})$ is stable if and only if its image $\iota(B)$ is irreducible as a representation of $\widetilde{Q}+\bar{Q}$.

For a $G_{\mathbf{d}}(\mathbf{V})$-invariant Zariski closed subset $Z$ of $\mathbf{M}_{\mathbf{Q}, \mathbf{d}}(\mathbf{V})$, let $Z^{\mathrm{s}}$ be the subset of all stable points in $Z$.

Proposition 3.2. The group $G_{\mathbf{d}}(\mathbf{V}) / \mathbb{C}^{\times}$acts freely and properly on $Z^{\mathrm{s}}$.

Proof. Note that the closed embedding $\iota$ defined in (3.1) is equivariant under the action of $G_{\mathbf{d}}(\mathbf{V}) \subset \mathrm{GL}\left(\mathbf{V}_{\mathbf{d}}\right)$. Hence the freeness of the $G_{\mathbf{d}}(\mathbf{V}) / \mathbb{C}^{\times}$-action on $Z^{\mathrm{s}}$ follows from that of the $\operatorname{GL}\left(\mathbf{V}_{\mathbf{d}}\right) / \mathbb{C}^{\times}$-action on $\operatorname{Rep}_{\widetilde{Q}+\bar{Q}}\left(\mathbf{V}_{\mathbf{d}}\right)^{\text {irr }}$ and

$$
\iota\left(\mathbf{M}_{\mathbf{Q}, \mathbf{d}}(\mathbf{V})^{\mathrm{s}}\right)=\iota\left(\mathbf{M}_{\mathbf{Q}, \mathbf{d}}(\mathbf{V})\right) \cap \operatorname{Rep}_{\widetilde{Q}+\overline{\mathbf{Q}}}\left(\mathbf{V}_{\mathbf{d}}\right)^{\mathrm{irr}},
$$

which we have already checked. Furthermore, the above implies that the embedding $Z^{\mathrm{s}} \hookrightarrow$ $\operatorname{Rep}_{\widetilde{Q}+\bar{Q}}\left(\mathbf{V}_{\mathbf{d}}\right)^{\text {irr }}$ induced from $\iota$ is closed. Consider the following commutative diagram:

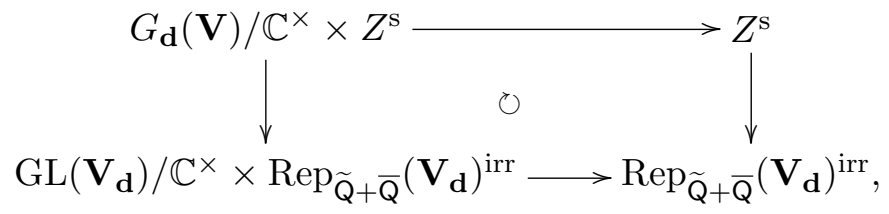

where the vertical arrows are the maps induced from $\iota$, and the horizonal arrows are the action maps $(g, x) \mapsto g \cdot x$. Since the bottle horizontal arrow is proper and both vertical arrows are closed, the properness of the top horizontal arrow follows from well-known basic properties of proper maps (see e.g. [11, Corollary 4.8]).

Definition 3.3. For $\lambda \in R^{\mathbf{d}}$ and $\mathbf{v} \in \mathbb{Z}_{\geq 0}^{I} \backslash\{0\}$, taking an $I$-graded $\mathbb{C}$-vector space $\mathbf{V}$ with $\operatorname{dim} \mathbf{V}=\mathbf{v}$ we define

$$
\mathcal{N}_{\mathbf{Q}, \mathbf{d}}^{\mathrm{s}}(\lambda, \mathbf{v}):=\mu_{\mathbf{d}}^{-1}\left(-\lambda \mathrm{Id}_{\mathbf{V}}\right)^{\mathrm{s}} / G_{\mathbf{d}}(\mathbf{V}),
$$

which we call the quiver variety with multiplicities.

We also use the following set-theoretical quotient:

$$
\mathcal{N}_{\mathbf{Q}, \mathbf{d}}^{\text {set }}(\lambda, \mathbf{v}):=\mu_{\mathbf{d}}^{-1}\left(-\lambda \operatorname{Id}_{\mathbf{V}}\right) / G_{\mathbf{d}}(\mathbf{V}) .
$$

It is clear from the definition that if $(\mathbf{Q}, \mathbf{d})$ is multiplicity-free, i.e., $d_{i}=1$ for all $i \in I$, then $\mathcal{N}_{\mathbf{Q}, \mathbf{d}}^{\mathbf{s}}(\lambda, \mathbf{v})$ coincides with the ordinal quiver variety $\mathcal{N}_{\mathbf{Q}}^{\mathbf{s}}(\zeta, \mathbf{v})$ with $\zeta_{i}=\operatorname{res}_{z=0} \lambda_{i}(z)$. Even when $\mathbf{d}$ is non-trivial, for simplicity, we often refer to $\mathcal{N}_{\mathbf{Q}, \mathbf{d}}^{\mathrm{s}}(\lambda, \mathbf{v})$ just as the 'quiver variety'.

\subsection{Properties}

Here we introduce some basic properties of quiver varieties with multiplicities.

First, we associate a symmetrizable Kac-Moody algebra to a quiver with multiplicities $(\mathbf{Q}, \mathbf{d})$. Let $\mathbf{A}=\left(a_{i j}\right)_{i, j \in I}$ be the adjacency matrix of the underlying graph of $\mathbf{Q}$ and set $\mathbf{D}:=\left(d_{i} \delta_{i j}\right)_{i, j \in I}$. Consider the generalized Cartan matrix

$$
\mathbf{C}=\left(c_{i j}\right)_{i, j \in I}:=2 \mathrm{Id}-\mathbf{A D} .
$$


Note that it is symmetrizable as $\mathbf{D C}=2 \mathbf{D}-\mathbf{D A D}$ is symmetric. Let

$$
\left(\mathfrak{g}(\mathbf{C}), \mathfrak{h},\left\{\alpha_{i}\right\}_{i \in I},\left\{\alpha_{i}^{\vee}\right\}_{i \in I}\right)
$$

be the corresponding Kac-Moody algebra with its Cartan subalgebra, simple roots and simple coroots. As usual we set

$$
Q:=\sum_{i \in I} \mathbb{Z} \alpha_{i}, \quad Q_{+}:=\sum_{i \in I} \mathbb{Z}_{\geq 0} \alpha_{i}
$$

The diagonal matrix $\mathbf{D}$ induces a non-degenerate invariant symmetric bilinear form $\left(\right.$, ) on $\mathfrak{h}^{*}$ satisfying

$$
\left(\alpha_{i}, \alpha_{j}\right)=d_{i} c_{i j}=2 d_{i} \delta_{i j}-d_{i} a_{i j} d_{j}, \quad i, j \in I .
$$

From now on, we regard a dimension vector $\mathbf{v} \in \mathbb{Z}_{\geq 0}^{I}$ of the quiver variety as an element of $Q_{+}$by

$$
\mathbb{Z}_{\geq 0}^{I} \stackrel{\simeq}{\longrightarrow} Q_{+}, \quad \mathbf{v}=\left(v_{i}\right)_{i \in I} \mapsto \sum_{i \in I} v_{i} \alpha_{i} .
$$

Let res: $R^{\mathbf{d}} \rightarrow \mathbb{C}^{I}$ be the map defined by

$$
\text { res: } \lambda=\left(\lambda_{i}(z)\right) \longmapsto\left(\underset{z=0}{\operatorname{res}} \lambda_{i}(z)\right),
$$

and for $(\mathbf{v}, \zeta) \in Q \times \mathbb{C}^{I}$, let $\mathbf{v} \cdot \zeta:=\sum_{i \in I} v_{i} \zeta_{i}$ be the scalar product.

\section{Proposition 3.4.}

(i) The quiver variety $\mathcal{N}_{\mathbf{Q}, \mathbf{d}}^{\mathrm{s}}(\lambda, \mathbf{v})$ is a holomorphic symplectic manifold of dimension $2-(\mathbf{v}, \mathbf{v})$ if it is nonempty.

(ii) If $\mathbf{v} \cdot \operatorname{res} \lambda \neq 0$, then $\mathcal{N}_{\mathbf{Q}, \mathbf{d}}^{\text {set }}(\lambda, \mathbf{v})=\varnothing$.

(iii) If two quivers $\mathrm{Q}_{1}, \mathrm{Q}_{2}$ have the same underlying graph, then the associated quiver varieties $\mathcal{N}_{\mathrm{Q}_{1}, \mathbf{d}}^{\mathrm{s}}(\lambda, \mathbf{v}), \mathcal{N}_{\mathrm{Q}_{2}, \mathbf{d}}^{\mathrm{s}}(\lambda, \mathbf{v})$ are symplectomorphic to each other.

Proof. (i) Assume that $\mathcal{N}_{\mathbf{Q}, \mathbf{d}}^{\mathrm{s}}(\lambda, \mathbf{v})$ is nonempty. Since the action of $G_{\mathbf{d}}(\mathbf{V}) / \mathbb{C}^{\times}$on the level set $\mu_{\mathbf{d}}^{-1}\left(-\lambda \mathrm{Id}_{\mathbf{V}}\right)^{\mathrm{s}}$ is free and proper, the Marsden-Weinstein reduction theorem implies that $\mathcal{N}_{Q, \mathbf{d}}^{\mathrm{s}}(\lambda, \mathbf{v})$ is a holomorphic symplectic manifold and

$$
\begin{aligned}
\operatorname{dim} \mathcal{N}_{\mathbf{Q}, \mathbf{d}}^{\mathrm{s}}(\lambda, \mathbf{v}) & =\operatorname{dim} \mathbf{M}_{\mathbf{Q}, \mathbf{d}}(\mathbf{V})-2 \operatorname{dim} G_{\mathbf{d}}(\mathbf{V}) / \mathbb{C}^{\times}=\sum_{i, j \in I} a_{i j} d_{i} v_{i} d_{j} v_{j}-2 \sum_{i \in I} d_{i} v_{i}^{2}+2 \\
& ={ }^{t} \mathbf{v D A D} \mathbf{D}-2^{t} \mathbf{v D} \mathbf{v}+2=2-(\mathbf{v}, \mathbf{v}) .
\end{aligned}
$$

(ii) Assume $\mathcal{N}_{\mathbf{Q}, \mathbf{d}}^{\text {set }}(\lambda, \mathbf{v}) \neq \varnothing$ and take a point $[B] \in \mathcal{N}_{\mathbf{Q}, \mathbf{d}}^{\text {set }}(\lambda, \mathbf{v})$. Then we have

$$
\sum_{k=1}^{d_{i}} \sum_{\substack{h \in H: \\ \mathrm{t}(h)=i}} \epsilon(h) \operatorname{tr}_{R_{d_{i}}}\left[B_{h} B_{\bar{h}} N_{i}^{k-1}\right] z^{-k}=-\lambda_{i}(z) \operatorname{Id}_{\mathbf{V}}
$$

for any $i \in I$. Taking res $\circ$ tr of both sides and sum over all $i$, we obtain

$$
\sum_{h \in H} \epsilon(h) \operatorname{tr}\left(B_{h} B_{\bar{h}}\right)=-\sum_{i \in I} v_{i} \underset{z=0}{\operatorname{res}} \lambda_{i}(z)=-\mathbf{v} \cdot \operatorname{res} \lambda .
$$


Here the left hand side is zero because

$$
\sum_{h \in H} \epsilon(h) \operatorname{tr}\left(B_{h} B_{\bar{h}}\right)=\sum_{h \in H} \epsilon(\bar{h}) \operatorname{tr}\left(B_{\bar{h}} B_{h}\right)=-\sum_{h \in H} \epsilon(h) \operatorname{tr}\left(B_{h} B_{\bar{h}}\right) .
$$

Hence $\mathbf{v} \cdot \operatorname{res} \lambda=0$.

(iii) By the assumption, we can identify the double quivers $\mathrm{Q}_{1}+\overline{\mathrm{Q}}_{1}$ and $\mathrm{Q}_{2}+\overline{\mathrm{Q}}_{2}$. Let $H$ be the set of arrows for them. Then both the sets of arrows $\Omega_{1}, \Omega_{2}$ for $\mathrm{Q}_{1}, \mathrm{Q}_{2}$ are subsets of $H$. Now the linear map $\mathbf{M}_{\mathrm{Q}_{1}, \mathbf{d}}(\mathbf{V}) \rightarrow \mathbf{M}_{\mathrm{Q}_{2}, \mathbf{d}}(\mathbf{V})=\mathbf{M}_{\mathrm{Q}_{1}, \mathbf{d}}(\mathbf{V})$ defined by

$$
B \mapsto B^{\prime}, \quad B_{h}^{\prime}:=\left\{\begin{aligned}
B_{h} & \text { if } h \in \Omega_{1} \cap \Omega_{2} \text { or } h \in \bar{\Omega}_{1} \cap \bar{\Omega}_{2}, \\
-B_{h} & \text { otherwise, }
\end{aligned}\right.
$$

induces a desired symplectomorphism $\mathcal{N}_{\mathrm{Q}_{1}, \mathbf{d}}^{\mathrm{s}}(\lambda, \mathbf{v}) \stackrel{\simeq}{\leftrightarrows} \mathcal{N}_{\mathrm{Q}_{2}, \mathbf{d}}^{\mathrm{s}}(\lambda, \mathbf{v})$.

Now fix $i \in I$ and set

$$
\widehat{V}_{i}:=\bigoplus_{\mathrm{t}(h)=i} V_{\mathrm{s}(h)} \otimes_{\mathbb{C}} R_{d_{\mathrm{s}(h)}} .
$$

Then using it we can decompose the vector space $\mathbf{M}_{\mathbf{Q}, \mathbf{d}}(\mathbf{V})$ as

$$
\mathbf{M}_{\mathbf{Q}, \mathbf{d}}(\mathbf{V})=\operatorname{Hom}\left(\widehat{V}_{i}, V_{i} \otimes_{\mathbb{C}} R_{d_{i}}\right) \oplus \operatorname{Hom}\left(V_{i} \otimes_{\mathbb{C}} R_{d_{i}}, \widehat{V}_{i}\right) \oplus \mathbf{M}_{\mathbf{Q}, \mathbf{d}}^{(i)}(\mathbf{V}),
$$

where

$$
\mathbf{M}_{\mathbf{Q}, \mathbf{d}}^{(i)}(\mathbf{V}):=\bigoplus_{\mathbf{t}(h), \mathbf{s}(h) \neq i} \operatorname{Hom}\left(V_{\mathbf{s}(h)} \otimes_{\mathbb{C}} R_{d_{\mathbf{s}(h)}}, V_{\mathbf{t}(h)} \otimes_{\mathbb{C}} R_{d_{\mathbf{t}(h)}}\right) .
$$

According to this decomposition, for a point $B \in \mathbf{M}_{\mathbf{Q}, \mathbf{d}}(\mathbf{V})$ we put

$$
\begin{aligned}
& B_{i \leftarrow}:=\left(\epsilon(h) B_{h}\right)_{\mathrm{t}(h)=i} \in \operatorname{Hom}\left(\widehat{V}_{i}, V_{i} \otimes_{\mathbb{C}} R_{d_{i}}\right), \\
& B_{\leftarrow i}:=\left(B_{\bar{h}}\right)_{\mathbf{t}(h)=i} \in \operatorname{Hom}\left(V_{i} \otimes_{\mathbb{C}} R_{d_{i}}, \widehat{V}_{i}\right), \\
& B_{\neq i}:=\left(B_{h}\right)_{\mathbf{t}(h), \mathbf{s}(h) \neq i} \in \mathbf{M}_{\mathbf{Q}, \mathbf{d}}^{(i)}(\mathbf{V}) .
\end{aligned}
$$

We regard these as coordinates for $B$ and write $B=\left(B_{i \leftarrow}, B_{\leftarrow i}, B_{\neq i}\right)$. Note that the symplectic form can be written as

$$
\omega=\operatorname{tr} \mathrm{d} B_{i \leftarrow} \wedge \mathrm{d} B_{\leftarrow i}+\frac{1}{2} \sum_{\mathrm{s}(h), \mathrm{t}(h) \neq i} \epsilon(h) \operatorname{tr} \mathrm{d} B_{h} \wedge \mathrm{d} B_{\bar{h}},
$$

and also the $i$-th component of the moment map can be written as

$$
\mu_{\mathbf{d}, i}(B)=\operatorname{pr}_{i}\left(B_{i \leftarrow} B_{\leftarrow i}\right)=\sum_{k=1}^{d_{i}} \operatorname{tr}_{R_{d_{i}}}\left[B_{i \leftarrow} B_{\leftarrow i} N_{i}^{k-1}\right] z^{-k} .
$$

Lemma 3.5. Fix $i \in I$ and suppose that $B$ satisfies at least one of the following two conditions:

(i) $B$ is stable and $\mathbf{v} \neq \alpha_{i}$;

(ii) the top coefficient $\operatorname{tr}_{R_{d_{i}}}\left(B_{i \leftarrow} B_{\leftarrow i} N_{i}^{d_{i}-1}\right)$ of $\operatorname{pr}_{i}\left(B_{i \leftarrow} B_{\leftarrow i}\right)$ is invertible.

Then $\left(B_{i \leftarrow}, B_{\leftarrow i}\right)$ satisfies

$\operatorname{Ker} B_{\leftarrow i} \cap \operatorname{Ker} N_{i}=0, \quad \operatorname{Im} B_{i \leftarrow}+\operatorname{Im} N_{i}=V_{i} \otimes_{\mathbb{C}} R_{d_{i}}$. 
Proof. First, assume (i) and set

$$
\begin{array}{ll}
\mathbf{S}=\bigoplus_{j \in I} S_{j}, & S_{j}:= \begin{cases}\operatorname{Ker} B_{\leftarrow i} \cap \operatorname{Ker} N_{i} & \text { if } j=i, \\
0 & \text { if } j \neq i,\end{cases} \\
\mathbf{T}=\bigoplus_{j \in I} T_{j}, & T_{j}:= \begin{cases}\operatorname{Im} B_{i \leftarrow}+\operatorname{Im} N_{i} & \text { if } j=i, \\
V_{j} \otimes_{\mathbb{C}} R_{d_{j}} & \text { if } j \neq i .\end{cases}
\end{array}
$$

Then both $\mathbf{S}$ and $\mathbf{T}$ are $B$-invariant and $N_{j}\left(S_{j}\right) \subset S_{j}, N_{j}\left(T_{j}\right) \subset T_{j}$ for all $j \in I$. Since $B$ is stable, we thus have $\mathbf{S}=0$ or $\mathbf{S}=\mathbf{V}_{\mathbf{d}}$, and $\mathbf{T}=0$ or $\mathbf{T}=\mathbf{V}_{\mathbf{d}}$. By the assumption $\mathbf{v} \neq \alpha_{i}$ and the definitions of $\mathbf{S}$ and $\mathbf{T}$, only the case $(\mathbf{S}, \mathbf{T})=\left(0, \mathbf{V}_{\mathbf{d}}\right)$ occurs. Hence $\left(B_{i \leftarrow}, B_{\leftarrow i}\right)$ satisfies (3.4).

Next assume (ii). Set $A(z)=\sum A_{k} z^{-k}:=\operatorname{pr}_{i}\left(B_{i \leftarrow} B_{\leftarrow i}\right)$ and

$$
\widetilde{A}:=\left(\begin{array}{cccc}
0 & 0 & \cdots & 0 \\
\vdots & \vdots & \ddots & \vdots \\
0 & 0 & \cdots & 0 \\
A_{d_{i}} & A_{d_{i}-1} & \cdots & A_{1}
\end{array}\right) \in \operatorname{End}_{\mathbb{C}}\left(V_{i} \otimes_{\mathbb{C}} \mathbb{C}^{d_{i}}\right)=\operatorname{End}_{\mathbb{C}}\left(V_{i} \otimes_{\mathbb{C}} R_{d_{i}}\right) .
$$

Then we have

$$
\operatorname{tr}_{R_{d_{i}}}\left(\widetilde{A} N^{k-1}\right)=A_{k}=\operatorname{tr}_{R_{d_{i}}}\left(B_{i \leftarrow} B_{\leftarrow i} N^{k-1}\right), \quad k=1,2, \ldots, d_{i},
$$

i.e., $\widetilde{A}-B_{i \leftarrow} B_{\leftarrow i} \in \operatorname{Ker} \operatorname{pr}_{i}$. Here, since the group $G_{d_{i}}\left(V_{i}\right)$ coincides with the centralizer of $N_{i}$ in $\mathrm{GL}_{\mathbb{C}}\left(V_{i} \otimes_{\mathbb{C}} R_{d_{i}}\right)$, we have $\operatorname{Ker~pr}_{i}=\operatorname{Imad}_{N_{i}}$. Hence there is $C \in \operatorname{End}_{\mathbb{C}}\left(V_{i} \otimes_{\mathbb{C}} R_{d_{i}}\right)$ such that

$$
B_{i \leftarrow} B_{\leftarrow i}=\widetilde{A}+\left[N_{i}, C\right] .
$$

Now note that both $\operatorname{Ker} N_{i}$ and Coker $N_{i}$ are naturally isomorphic to $V_{i}$, and the natural injection $\iota:$ Ker $N_{i} \rightarrow V_{i} \otimes_{\mathbb{C}} R_{d_{i}}$ and projection $\pi: V_{i} \otimes_{\mathbb{C}} R_{d_{i}} \rightarrow$ Coker $N_{i}$ can be respectively identified with the following block matrices:

$$
\left(\begin{array}{c}
\mathrm{Id}_{V} \\
0 \\
\vdots \\
0
\end{array}\right): V_{i} \rightarrow V_{i} \otimes_{\mathbb{C}} R_{d_{i}}, \quad\left(\begin{array}{lllll}
0 & 0 & \cdots & 0 & \mathrm{Id}_{V}
\end{array}\right): V_{i} \otimes_{\mathbb{C}} R_{d_{i}} \rightarrow V_{i} .
$$

Thus we have

$$
\pi B_{i \leftarrow} B_{\leftarrow i \iota}=\pi\left(\widetilde{A}+\left[N_{i}, C\right]\right) \iota=\pi \widetilde{A} \iota=A_{d_{i}} .
$$

By the assumption $A_{d_{i}}$ is invertible. Hence $\pi B_{i \leftarrow}$ is surjective and $B_{\leftarrow i} \iota$ is injective.

The following lemma is a consequence of results obtained in [31]:

Lemma 3.6. Suppose that the set

$$
\begin{array}{r}
Z_{i}:=\left\{\left(B_{i \leftarrow}, B_{\leftarrow i}\right) \in \operatorname{Hom}\left(\widehat{V}_{i}, V_{i} \otimes_{\mathbb{C}} R_{d_{i}}\right) \oplus \operatorname{Hom}\left(V_{i} \otimes_{\mathbb{C}} R_{d_{i}}, \widehat{V}_{i}\right) \mid\right. \\
\left.\operatorname{pr}_{i}\left(B_{i \leftarrow} B_{\leftarrow i}\right)=-\lambda_{i}(z) \operatorname{Id}_{V_{i}}, \quad\left(B_{i \leftarrow}, B_{\leftarrow i}\right) \text { satisfies }(3.4)\right\}
\end{array}
$$

is nonempty. Then the quotient of it modulo the action of $G_{d_{i}}\left(V_{i}\right)$ is a smooth complex manifold having a symplectic structure induced from $\operatorname{tr} \mathrm{d} B_{i \leftarrow} \wedge \mathrm{d} B_{\leftarrow i}$, and is symplectomorphic to a $G_{d_{i}}\left(\widehat{V}_{i}\right)$ coadjoint orbit via the map given by

$$
\Phi_{i}: \quad\left(B_{i \leftarrow}, B_{\leftarrow i}\right) \longmapsto-B_{\leftarrow i}\left(z-N_{i}\right)^{-1} B_{i \leftarrow} \in \mathfrak{g}_{d_{i}}^{*}\left(\widehat{V}_{i}\right) .
$$


Proof. Take any point $\left(B_{i \leftarrow}, B_{\leftarrow i}\right)$ in the above set and let $\mathcal{O}$ be the $G_{d_{i}}\left(\widehat{V}_{i}\right)$-coadjoint orbit through $\Phi_{i}\left(B_{i \leftarrow}, B_{\leftarrow i}\right)$. By Proposition 4, (a), Theorem 6 and Lemma 3 in [31], there exist

- a finite-dimensional $\mathbb{C}$-vector space $W$;

- a nilpotent endomorphism $N \in \operatorname{End}(W)$ with $N^{d_{i}}=0$;

- a coadjoint orbit $\mathcal{O}_{N} \subset\left(\operatorname{Lie} G_{N}\right)^{*}$ of the centralizer $G_{N} \subset \mathrm{GL}(W)$ of $N$,

such that the quotient modulo the natural $G_{N}$-action of the set

$$
\left\{\begin{array}{l|l}
(Y, X) \in \operatorname{Hom}\left(\widehat{V}_{i}, W\right) \oplus \operatorname{Hom}\left(W, \widehat{V}_{i}\right) \mid \begin{array}{l}
\operatorname{pr}_{N}(Y X) \in \mathcal{O}_{N}, \\
\operatorname{Ker} X \cap \operatorname{Ker} N=0, \quad \operatorname{Im} Y+\operatorname{Im} N=\widehat{V}_{i}
\end{array}
\end{array}\right\},
$$

where $\operatorname{pr}_{N}$ is the transpose of the inclusion Lie $G_{N} \hookrightarrow \mathfrak{g l}(W)$, is a smooth manifold having a symplectic structure induced from $\operatorname{tr} \mathrm{d} X \wedge \mathrm{d} Y$, and is symplectomorphic to $\mathcal{O}$ via the map $(Y, X) \mapsto X\left(z \operatorname{Id}_{W}-N\right)^{-1} Y$. Note that if $W=V_{i} \otimes_{\mathbb{C}} R_{d_{i}}, N=N_{i}$ and $\mathcal{O}_{N}$ is a single element $\lambda_{i}(z) \operatorname{Id}_{V_{i}}$, then we obtain the result by the coordinate change $(Y, X)=\left(B_{i \leftarrow},-B_{\leftarrow i}\right)$. Indeed, this is the case thanks to Proposition 4, (c) and Theorem 6 (the uniqueness assertion) in [31].

Note that in the above lemma, the assumption $Z_{i} \neq \varnothing \operatorname{implies} \operatorname{dim} V_{i} \leq \operatorname{dim} \widehat{V}_{i}$; indeed, if $\left(B_{i \leftarrow}, B_{\leftarrow i}\right) \in Z_{i}$, then $\left.B_{\leftarrow i}\right|_{\operatorname{Ker} N_{i}}$ is injective by condition (3.4), and hence

$$
\operatorname{dim} V_{i}=\operatorname{dim} \operatorname{Ker} N_{i}=\operatorname{rank}\left(\left.B_{\leftarrow i}\right|_{\operatorname{Ker}} N_{i}\right) \leq \operatorname{dim} \widehat{V}_{i} .
$$

The following lemma tells us that if the top coefficient of $\lambda_{i}(z)$ is nonzero, then the converse is true and the corresponding coadjoint orbit can be explicitly described:

Lemma 3.7. Suppose $\operatorname{dim} V_{i} \leq \operatorname{dim} \widehat{V}_{i}$ and that the top coefficient $\lambda_{i, d_{i}}$ of $\lambda_{i}(z)$ is nonzero. Then the set $Z_{i}$ in Lemma 3.6 is nonempty and the coadjoint orbit contains an element of the form

$$
\Lambda_{i}(z)=\left(\begin{array}{cc}
\lambda_{i}(z) \operatorname{Id}_{V_{i}} & 0 \\
0 & 0 \operatorname{Id}_{V_{i}^{\prime}}
\end{array}\right)
$$

where $V_{i}$ is regarded as a subspace of $\widehat{V}_{i}$ and $V_{i}^{\prime} \subset \widehat{V}_{i}$ is a complement of it.

Proof. Suppose $\operatorname{dim} V_{i} \leq \operatorname{dim} \widehat{V}_{i}$ and that the top coefficient of $\lambda_{i}(z)$ is nonzero. We set

$$
\begin{aligned}
B_{i \leftarrow}:= & \left(\begin{array}{cc}
0 & 0 \\
\vdots & \vdots \\
0 & 0 \\
\operatorname{Id}_{V_{i}} & 0
\end{array}\right): \widehat{V}_{i}=V_{i} \oplus V_{i}^{\prime} \rightarrow V_{i} \otimes_{\mathbb{C}} R_{d_{i}}, \\
B_{\leftarrow i} & :=-\left(\begin{array}{ccc}
\lambda_{i, d_{i}} \operatorname{Id}_{V_{i}} & \cdots & \lambda_{i, 1} \operatorname{Id}_{V_{i}} \\
0 & \cdots & 0
\end{array}\right): V_{i} \otimes_{\mathbb{C}} R_{d_{i}} \rightarrow \widehat{V}_{i},
\end{aligned}
$$

where $\lambda_{i, k}$ denotes the coefficient in $\lambda_{i}(z)$ of $z^{-k}$. Then we have

$$
\operatorname{tr}_{R_{d_{i}}}\left(B_{i \leftarrow} B_{\leftarrow i} N_{i}^{k-1}\right)=-\lambda_{i, k} \operatorname{Id}_{V_{i}}, \quad k=1,2, \ldots, d_{i},
$$

i.e., $\operatorname{pr}_{i}\left(B_{i \leftarrow} B_{\leftarrow i}\right)=-\lambda_{i}(z) \operatorname{Id}_{V_{i}}$. The assumption $\lambda_{i, d_{i}} \neq 0$ and Lemma 3.5 imply that $\left(B_{i \leftarrow}, B_{\leftarrow i}\right)$ satisfies (3.4). Hence $\left(B_{i \leftarrow}, B_{\leftarrow i}\right) \in Z_{i}$. Moreover we have

$$
\begin{aligned}
\Phi_{i}\left(B_{i \leftarrow}, B_{\leftarrow i}\right)=-B_{\leftarrow i}\left(z-N_{i}\right)^{-1} B_{i \leftarrow} & =-\sum_{k=1} B_{\leftarrow i} N_{i}^{k-1} B_{i \leftarrow} z^{-k} \\
& =\sum_{k=1}\left(\begin{array}{cc}
\lambda_{i, k} \operatorname{Id}_{V_{i}} & 0 \\
0 & 0 \operatorname{Id}_{V_{i}^{\prime}}
\end{array}\right) z^{-k}=\Lambda_{i}(z) .
\end{aligned}
$$




\section{Reflection functor}

In this section we construct reflection functors for quiver varieties with multiplicities.

\subsection{Main theorem}

Recall that the Weyl group $W(\mathbf{C})$ of the Kac-Moody algebra $\mathfrak{g}(\mathbf{C})$ is the subgroup of GL( $\left.\mathfrak{h}^{*}\right)$ generated by the simple reflections

$$
s_{i}(\beta):=\beta-\left\langle\beta, \alpha_{i}^{\vee}\right\rangle \alpha_{i}=\beta-\frac{2\left(\beta, \alpha_{i}\right)}{\left(\alpha_{i}, \alpha_{i}\right)} \alpha_{i}, \quad i \in I, \quad \beta \in \mathfrak{h}^{*} .
$$

The fundamental relations for the generators $s_{i}, i \in I$ are

$$
s_{i}^{2}=\mathrm{Id}, \quad\left(s_{i} s_{j}\right)^{m_{i j}}=\mathrm{Id}, \quad i, j \in I, \quad i \neq j,
$$

where the numbers $m_{i j}$ are determined from $c_{i j} c_{j i}$ as the table below (we use the convention $r^{\infty}=$ Id for any $r$ )

\begin{tabular}{|c|c|c|c|c|c|}
\hline$c_{i j} c_{j i}$ & 0 & 1 & 2 & 3 & $\geq 4$ \\
\hline$m_{i j}$ & 2 & 3 & 4 & 6 & $\infty$ \\
\hline
\end{tabular}

We will define a $W(\mathbf{C})$-action on the parameter space $R^{\mathbf{d}} \times Q$ for the quiver variety. The action on the second component $Q$ is given by just the restriction of the standard action on $\mathfrak{h}^{*}$, namely,

$$
s_{i}: \mathbf{v}=\sum_{i \in I} v_{i} \alpha_{i} \longmapsto \mathbf{v}-\left\langle\mathbf{v}, \alpha_{i}^{\vee}\right\rangle \alpha_{i}=\mathbf{v}-\sum_{j \in I} c_{i j} v_{j} \alpha_{i}
$$

The action on the first component $R^{\mathbf{d}}$ is unusual. We define $r_{i} \in \operatorname{GL}\left(R^{\mathbf{d}}\right)$ by

$$
r_{i}(\lambda)=\lambda^{\prime} \equiv\left(\lambda_{j}^{\prime}(z)\right), \quad \lambda_{j}^{\prime}(z):= \begin{cases}-\lambda_{i}(z) & \text { if } j=i \\ \lambda_{j}(z)-z^{-1} c_{i j} \underset{z=0}{\operatorname{ess}} \lambda_{i}(z) & \text { if } j \neq i\end{cases}
$$

Lemma 4.1. The above $r_{i}, i \in I$ satisfy relations (4.1).

Proof. The relations $r_{i}^{2}=\mathrm{Id}, i \in I$ are obvious. To check the relation $\left(r_{i} r_{j}\right)^{m_{i j}}=\mathrm{Id}$ for $i \neq j$, first note that the transpose of $s_{i}: Q \rightarrow Q$ relative to the scalar product is given by

$$
{ }^{t} s_{i}: \mathbb{C}^{I} \rightarrow \mathbb{C}^{I}, \quad{ }^{t} s_{i}(\zeta)=\zeta-\zeta_{i} \sum_{j \in I} c_{i j} \alpha_{j}
$$

Now let $\lambda \in R^{\mathbf{d}}$. We decompose it as

$$
\lambda=\lambda^{0}+\operatorname{res}(\lambda) z^{-1}, \quad \operatorname{res} \lambda^{0}=0 .
$$

Then we easily see that

$$
r_{i}\left(\operatorname{res}(\lambda) z^{-1}\right)={ }^{t} s_{i}(\operatorname{res}(\lambda)) z^{-1},
$$

and hence that

$$
\left(r_{i} r_{j}\right)^{m_{i j}}(\lambda)=\left(r_{i} r_{j}\right)^{m_{i j}}\left(\lambda^{0}\right)+\operatorname{res}(\lambda) z^{-1} .
$$


Therefore we may assume that $\operatorname{res} \lambda=0$. Set $\lambda^{\prime} \equiv\left(\lambda_{k}^{\prime}(z)\right):=\left(r_{i} r_{j}\right)^{m_{i j}}(\lambda)$. Then we have

$$
\lambda_{k}^{\prime}(z)= \begin{cases}(-1)^{m_{i j}} \lambda_{k}(z) & \text { if } k=i, j \\ \lambda_{k}(z) & \text { if } k \neq i, j .\end{cases}
$$

If $m_{i j}$ is odd, by the definition we have $c_{i j} c_{j i}=1$. In particular, $i \neq j$ and

$$
a_{i j} d_{j} a_{j i} d_{i}=c_{i j} c_{j i}=1 .
$$

This implies $d_{i}=d_{j}=1$ and hence that $\lambda_{i}(z)=\lambda_{j}(z)=0$.

The main result of this section is as follows:

Theorem 4.2. Let $\lambda=\left(\lambda_{i}(z)\right) \in R^{\mathbf{d}}$ and suppose that the top coefficient $\lambda_{i, d_{i}}$ of $\lambda_{i}(z)$ for fixed $i \in I$ is nonzero. Then there exists a bijection

$$
\mathcal{F}_{i}: \quad \mathcal{N}_{\mathbf{Q}, \mathbf{d}}^{\mathrm{set}}(\lambda, \mathbf{v}) \stackrel{\simeq}{\longrightarrow} \mathcal{N}_{\mathbf{Q}, \mathbf{d}}^{\text {set }}\left(r_{i}(\lambda), s_{i}(\mathbf{v})\right)
$$

such that $\mathcal{F}_{i}^{2}=\mathrm{Id}$ and the restriction gives a symplectomorphism

$$
\mathcal{F}_{i}: \quad \mathcal{N}_{\mathbf{Q}, \mathbf{d}}^{\mathrm{s}}(\lambda, \mathbf{v}) \stackrel{\simeq}{\longrightarrow} \mathcal{N}_{\mathrm{Q}, \mathbf{d}}^{\mathrm{s}}\left(r_{i}(\lambda), s_{i}(\mathbf{v})\right) .
$$

We call the above map $\mathcal{F}_{i}$ the $i$-th reflection functor.

\subsection{Proof of the main theorem}

Fix $i \in I$ and suppose that the top coefficient $\lambda_{i, d_{i}}$ of $\lambda_{i}(z)$ is nonzero. Recall the decomposition (3.2) of $\mathbf{M}_{\mathbf{Q}, \mathbf{d}}(\mathbf{V})$ :

$$
\mathbf{M}_{\mathbf{Q}, \mathbf{d}}(\mathbf{V})=\operatorname{Hom}\left(\widehat{V}_{i}, V_{i} \otimes_{\mathbb{C}} R_{d_{i}}\right) \oplus \operatorname{Hom}\left(V_{i} \otimes_{\mathbb{C}} R_{d_{i}}, \widehat{V}_{i}\right) \oplus \mathbf{M}_{\mathbf{Q}, \mathbf{d}}^{(i)}(\mathbf{V}),
$$

and the set $Z_{i}$ given in Lemma 3.6. Lemma 3.5 and the assumption $\lambda_{i, d_{i}} \neq 0$ imply that any $B=\left(B_{i \leftarrow}, B_{\leftarrow i}, B_{\neq i}\right) \in \mu_{\mathbf{d}, i}^{-1}\left(-\lambda_{i}(z) \operatorname{Id}_{V_{i}}\right)$ satisfies condition (3.4). Thus we have

$$
\mu_{\mathbf{d}, i}^{-1}\left(-\lambda_{i}(z) \operatorname{Id}_{V_{i}}\right)=Z_{i} \times \mathbf{M}_{\mathbf{Q}, \mathbf{d}}^{(i)}(\mathbf{V}) .
$$

By Lemma 3.7, it is nonempty if and only if

$$
v_{i} \leq \operatorname{dim} \widehat{V}_{i}=\sum_{j} a_{i j} d_{j} v_{j}=2 v_{i}-\sum_{j} c_{i j} v_{j}
$$

i.e., the $i$-th component of $s_{i}(\mathbf{v})$ is non-negative. We assume this condition, because otherwise both $\mathcal{N}_{\mathbf{Q}, \mathbf{d}}^{\text {set }}(\lambda, \mathbf{v})$ and $\mathcal{N}_{\mathbf{Q}, \mathbf{d}}^{\text {set }}\left(r_{i}(\lambda), s_{i}(\mathbf{v})\right)$ are empty (since $\left.s_{i}(\mathbf{v}) \notin \mathbb{Z}_{\geq 0}^{I}\right)$. Fix a $\mathbb{C}$-vector space $V_{i}^{\prime}$ of dimension $\operatorname{dim} \widehat{V}_{i}-\operatorname{dim} V_{i}$ and an identification $\widehat{V}_{i}=V_{i} \oplus V_{i}^{\prime}$. As the group $G_{d_{i}}\left(V_{i}\right)$ acts trivially on $\mathbf{M}_{\mathbf{Q}, \mathbf{d}}^{(i)}(\mathbf{V})$, Lemmas 3.6 and 3.7 imply that

$$
\mu_{\mathbf{d}, i}^{-1}\left(-\lambda_{i}(z) \operatorname{Id}_{V_{i}}\right) / G_{d_{i}}\left(V_{i}\right)=Z_{i} / G_{d_{i}}\left(V_{i}\right) \times \mathbf{M}_{\mathbf{Q}, \mathbf{d}}^{(i)}(\mathbf{V}) \simeq \mathcal{O} \times \mathbf{M}_{\mathbf{Q}, \mathbf{d}}^{(i)}(\mathbf{V}),
$$

where $\mathcal{O}$ is the $G_{d_{i}}\left(\widehat{V}_{i}\right)$-coadjoint orbit through

$$
\Lambda(z)=\left(\begin{array}{cc}
\lambda_{i}(z) \operatorname{Id}_{V_{i}} & 0 \\
0 & 0 \operatorname{Id}_{V_{i}^{\prime}}
\end{array}\right)
$$


Now let us define an $I$-graded $\mathbb{C}$-vector space $\mathbf{V}^{\prime}$ with $\operatorname{dim} \mathbf{V}^{\prime}=s_{i}(\mathbf{v})$ by

$$
\mathbf{V}^{\prime}=\bigoplus_{j \in I} V_{j}^{\prime}, \quad V_{j}^{\prime}:= \begin{cases}V_{i}^{\prime} & \text { if } j=i \\ V_{j} & \text { if } j \neq i\end{cases}
$$

and consider the associated symplectic vector space $\mathbf{M}_{\mathbf{Q}, \mathbf{d}}\left(\mathbf{V}^{\prime}\right)$. Note that $\widehat{V}_{i}^{\prime}=\widehat{V}_{i}$. Thus by interchanging the roles of $\mathbf{V}$ and $\mathbf{V}^{\prime}, \lambda_{i}$ and $-\lambda_{i}$ in Lemmas 3.6 and 3.7, we obtain an isomorphism

$$
\mu_{\mathbf{d}, i}^{-1}\left(\lambda_{i}(z) \operatorname{Id}_{V_{i}^{\prime}}\right) / G_{d_{i}}\left(V_{i}^{\prime}\right) \simeq \mathcal{O}^{\prime} \times \mathbf{M}_{\mathbf{Q}, \mathbf{d}}^{(i)}\left(\mathbf{V}^{\prime}\right)=\mathcal{O}^{\prime} \times \mathbf{M}_{\mathbf{Q}, \mathbf{d}}^{(i)}(\mathbf{V}),
$$

where $\mathcal{O}^{\prime}$ is the $G_{d_{i}}\left(\widehat{V}_{i}\right)$-coadjoint orbit through

$$
\left(\begin{array}{cc}
0 \operatorname{Id}_{V_{i}} & 0 \\
0 & -\lambda_{i}(z) \operatorname{Id}_{V_{i}^{\prime}}
\end{array}\right)=\Lambda(z)-\lambda_{i}(z) \operatorname{Id}_{\widehat{V}_{i}}
$$

i.e., $\mathcal{O}^{\prime}=\mathcal{O}-\lambda_{i}(z) \operatorname{Id}_{\widehat{V}_{i}}$. Hence the scalar shift $\mathcal{O} \stackrel{\simeq}{\longrightarrow} \mathcal{O}-\lambda_{i}(z) \operatorname{Id}_{\widehat{V}_{i}}$ induces an isomorphism

$$
\widetilde{\mathcal{F}}_{i}: \quad \mu_{\mathbf{d}, i}^{-1}\left(-\lambda_{i}(z) \operatorname{Id}_{V_{i}}\right) / G_{d_{i}}\left(V_{i}\right) \stackrel{\simeq}{\longrightarrow} \mu_{\mathbf{d}, i}^{-1}\left(\lambda_{i}(z) \operatorname{Id}_{V_{i}^{\prime}}\right) / G_{d_{i}}\left(V_{i}^{\prime}\right)
$$

which is characterized as follows: if

$$
\widetilde{\mathcal{F}}_{i}[B]=\left[B^{\prime}\right], \quad B=\left(B_{i \leftarrow}, B_{\leftarrow i}, B_{\neq i}\right), \quad B^{\prime}=\left(B_{i \leftarrow}^{\prime}, B_{\leftarrow i}^{\prime}, B_{\neq i}^{\prime}\right),
$$

one has

$$
\begin{aligned}
& B_{\neq i}=B_{\neq i}^{\prime}, \\
& -B_{\leftarrow i}^{\prime}\left(z-N_{i}^{\prime}\right)^{-1} B_{i \leftarrow}^{\prime}=-B_{\leftarrow i}\left(z-N_{i}\right)^{-1} B_{i \leftarrow}-\lambda_{i}(z) \operatorname{Id}_{\widehat{V}_{i}},
\end{aligned}
$$

where $N_{i}^{\prime}:=\operatorname{Id}_{V_{i}^{\prime}} \otimes_{\mathbb{C}} J_{d_{i}} \in \operatorname{End}_{\mathbb{C}}\left(V_{i}^{\prime} \otimes_{\mathbb{C}} R_{d_{i}}\right)$. Note that

$$
\operatorname{Ker} B_{\leftarrow i}^{\prime} \cap \operatorname{Ker} N_{i}^{\prime}=0, \quad \operatorname{Im} B_{i \leftarrow}^{\prime}+\operatorname{Im} N_{i}^{\prime}=V_{i}^{\prime} \otimes_{\mathbb{C}} R_{d_{i}}
$$

by Lemma 3.5.

Lemma 4.3. If $\mu_{\mathbf{d}}(B)=-\lambda \operatorname{Id}_{\mathbf{V}}$, then $\mu_{\mathbf{d}}\left(B^{\prime}\right)=-r_{i}(\lambda) \operatorname{Id}_{\mathbf{V}^{\prime}}$.

Proof. Let $\lambda^{\prime}=\left(\lambda_{j}^{\prime}(z)\right):=r_{i}(\lambda)$. The identity $\mu_{\mathbf{d}, i}\left(B^{\prime}\right)=\lambda_{i}(z) \operatorname{Id}_{V_{i}^{\prime}}$ is clear from the construction. We check $\mu_{\mathbf{d}, j}\left(B^{\prime}\right)=-\lambda_{j}^{\prime}(z) \operatorname{Id}_{V_{j}^{\prime}}$ for $j \neq i$. Taking the residue of both sides of (4.3), we have

$$
B_{\leftarrow i}^{\prime} B_{i \leftarrow}^{\prime}=B_{\leftarrow i} B_{i \leftarrow}+\lambda_{i, 1} \operatorname{Id}_{\widehat{V}_{i}},
$$

which implies that

$$
\epsilon(h) B_{\bar{h}}^{\prime} B_{h}^{\prime}=\epsilon(h) B_{\bar{h}} B_{h}+\lambda_{i, 1} \operatorname{Id}_{V_{\mathrm{s}(h)}} \quad \text { if } \quad \mathrm{t}(h)=i .
$$

On the other hand, (4.2) means that $B_{h}^{\prime}=B_{h}$ whenever $\mathrm{t}(h), \mathrm{s}(h) \neq i$. Thus for $j \neq i$, we obtain

$$
\begin{aligned}
\sum_{\mathrm{t}(h)=j} \epsilon(h) B_{h}^{\prime} B_{\bar{h}}^{\prime} & =\sum_{h: i \rightarrow j} \epsilon(h) B_{h}^{\prime} B_{\bar{h}}^{\prime}+\sum_{\mathrm{t}(h)=j, \mathbf{s}(h) \neq i} \epsilon(h) B_{h}^{\prime} B_{\bar{h}}^{\prime} \\
& =\sum_{h: i \rightarrow j}\left(\epsilon(h) B_{h} B_{\bar{h}}-\lambda_{i, 1} \operatorname{Id}_{V_{j}}\right)+\sum_{\mathrm{t}(h)=j, \mathbf{s}(h) \neq i} \epsilon(h) B_{h} B_{\bar{h}}
\end{aligned}
$$




$$
=\sum_{\mathbf{t}(h)=j} \epsilon(h) B_{h} B_{\bar{h}}-a_{i j} \lambda_{i, 1} \operatorname{Id}_{V_{j}}
$$

Note that

$$
\operatorname{pr}_{j}\left(\operatorname{Id}_{V_{j}}\right)=\sum_{k=1}^{d_{j}} \operatorname{tr}_{R_{d_{j}}}\left(N_{j}^{k-1}\right) z^{-k}=d_{j} \operatorname{Id}_{V_{j}} z^{-1}
$$

Therefore the image under $\mathrm{pr}_{j}$ of both sides of (4.5) gives

$$
\mu_{\mathbf{d}, j}\left(B^{\prime}\right)=\mu_{\mathbf{d}, j}(B)-a_{i j} \lambda_{i, 1} \operatorname{pr}_{i}\left(\operatorname{Id}_{V_{j}}\right)=\mu_{\mathbf{d}, j}(B)+c_{i j} \lambda_{i, 1} \operatorname{Id}_{V_{j}} z^{-1} .
$$

The result follows.

Lemma 4.4. If $B$ is stable, then so is $B^{\prime}$.

Proof. Suppose that there exists a $B^{\prime}$-invariant subspace $\mathbf{S}^{\prime}=\bigoplus_{j} S_{j}^{\prime} \subset \mathbf{V}_{\mathbf{d}}^{\prime}$ such that $N_{j}^{\prime}\left(S_{j}^{\prime}\right) \subset S_{j}^{\prime}$. We define an $I$-graded subspace $\mathbf{S}=\bigoplus_{j} S_{j}$ of $\mathbf{V}_{\mathbf{d}}$ by

$$
S_{j}:= \begin{cases}\sum_{k=1}^{d_{i}} N_{i}^{k-1} B_{i \leftarrow}\left(\widehat{S}_{i}^{\prime}\right) & \text { if } j=i, \\ S_{j}^{\prime} & \text { if } j \neq i,\end{cases}
$$

where $\widehat{S}_{i}^{\prime}:=\bigoplus_{\mathrm{t}(h)=i} S_{\mathrm{s}(h)}^{\prime}=\widehat{S}_{i}$. Then $B_{i \leftarrow}\left(\widehat{S}_{i}\right) \subset S_{i}$ and

$$
B_{\leftarrow i}\left(S_{i}\right)=\sum_{k=1}^{d_{i}} B_{\leftarrow i} N_{i}^{k-1} B_{i \leftarrow}\left(\widehat{S}_{i}^{\prime}\right)=\sum_{k=1}^{d_{i}}\left(B_{\leftarrow i}^{\prime}\left(N_{i}^{\prime}\right)^{k-1} B_{i \leftarrow}^{\prime}-\lambda_{i, k}\right)\left(\widehat{S}_{i}^{\prime}\right) \subset \widehat{S}_{i}^{\prime}=\widehat{S}_{i} .
$$

Hence $\mathbf{S}$ is $B$-invariant. Clearly $N_{j}\left(S_{j}\right) \subset S_{j}$ for all $j \in I$. Therefore the stability condition for $B$ implies that $\mathbf{S}=0$ or $\mathbf{S}=\mathbf{V}_{\mathbf{d}}$. First, assume $\mathbf{S}=0$. Then $S_{j}^{\prime}=S_{j}=0$ for $j \neq i$, and hence $B_{\leftarrow i}^{\prime}\left(S_{i}^{\prime}\right) \subset \widehat{S}_{i}^{\prime}=0$, i.e., $S_{i}^{\prime} \subset \operatorname{Ker} B_{\leftarrow i}^{\prime}$. If $S_{i}^{\prime}$ is nonzero, then the kernel of the restriction $\left.N_{i}^{\prime}\right|_{S_{i}^{\prime}}$ is nonzero because it is nilpotent. However it implies $\operatorname{Ker} B_{\leftarrow i}^{\prime} \cap \operatorname{Ker} N_{i}^{\prime} \neq 0$, which contradicts to (4.4). Hence $S_{i}^{\prime}=0$. Next assume $\mathbf{S}=\mathbf{V}_{\mathbf{d}}$. Then $S_{j}^{\prime}=S_{j}=V_{j} \otimes_{\mathbb{C}} R_{d_{j}}$ for $j \neq i$, and hence $S_{i}^{\prime} \supset B_{i \leftarrow}^{\prime}\left(\widehat{S}_{i}^{\prime}\right)=\operatorname{Im} B_{i \leftarrow}^{\prime}$. If $V_{i}^{\prime} / S_{i}^{\prime}$ is nonzero, then the endomorphism of $V_{i}^{\prime} / S_{i}^{\prime}$ induced from $N_{i}^{\prime}$ has a nonzero cokernel because it is nilpotent. However it implies $\operatorname{Im} B_{i \leftarrow}^{\prime}+\operatorname{Im} N_{i}^{\prime} \neq V_{i}^{\prime} \otimes_{\mathbb{C}} R_{d_{i}}$, which contradicts to (4.4). Hence $S_{i}^{\prime}=V_{i}^{\prime} \otimes_{\mathbb{C}} R_{d_{i}}$.

Proof of Theorem 4.2. As the map $\widetilde{\mathcal{F}}_{i}$ is clearly $\prod_{j \neq i} G_{d_{j}}\left(V_{j}\right)$-equivariant, Lemma 4.3 implies that it induces a bijection

$$
\mathcal{F}_{i}: \quad \mathcal{N}_{\mathbf{Q}, \mathbf{d}}^{\text {set }}(\lambda, \mathbf{v}) \rightarrow \mathcal{N}_{\mathbf{Q}, \mathbf{d}}^{\text {set }}\left(r_{i}(\lambda), s_{i}(\mathbf{v})\right), \quad[B] \mapsto\left[B^{\prime}\right],
$$

which preserves the stability by Lemma 4.4. We easily obtain the relation $\mathcal{F}_{i}^{2}=\mathrm{Id}$ by noting that $\mathcal{F}_{i}$ is induced from the scalar shift $\mathcal{O} \rightarrow \mathcal{O}^{\prime}=\mathcal{O}-\lambda_{i}(z) \operatorname{Id}_{\widehat{V}_{i}}$ and the $i$-th component of $r_{i}(\lambda)$ is $-\lambda_{i}$. Consider the restriction

$$
\mathcal{F}_{i}: \quad \mathcal{N}_{\mathbf{Q}, \mathbf{d}}^{\mathrm{s}}(\lambda, \mathbf{v}) \rightarrow \mathcal{N}_{\mathbf{Q}, \mathbf{d}}^{\mathrm{s}}\left(r_{i}(\lambda), s_{i}(\mathbf{v})\right), \quad[B] \mapsto\left[B^{\prime}\right] .
$$

By Lemma 3.6 and (4.3), we have

$$
\operatorname{tr} \mathrm{d} B_{i \leftarrow} \wedge \mathrm{d} B_{\leftarrow i}=\operatorname{tr} \mathrm{d} B_{i \leftarrow}^{\prime} \wedge \mathrm{d} B_{\leftarrow i}^{\prime},
$$

because the scalar shift $\mathcal{O} \rightarrow \mathcal{O}^{\prime}$ is a symplectomorphism. Substituting it and (4.2) into (3.3), we see that the above map $\mathcal{F}_{i}$ is a symplectomorphism. 
Remark 4.5. It is clear from (4.2), (4.3) and (4.4) that if $d_{j}=1$ for all $j \in I$, then $\mathcal{F}_{i}$ coincides with the original $i$-th reflection functor for quiver varieties (see conditions (a), (b1) and (c) in $[18$, Section 3]).

Remark 4.6. It is known (see e.g. [19]) that if $d_{i}=1$ for all $i \in I$, then the reflection functors $\mathcal{F}_{i}$ satisfy relations (4.1). We expect that this fact is true for any $(\mathbf{Q}, \mathbf{d})$.

\subsection{Application}

In this subsection we introduce a basic application of reflection functors.

Lemma 4.7. Let $(\lambda, \mathbf{v}) \in R^{\mathbf{d}} \times Q_{+}, i \in I$. Suppose that the top coefficient of $\lambda_{i}(z)$ is zero and $\mathbf{v} \neq \alpha_{i}$. Then $\mathcal{N}_{\mathbf{Q}, \mathbf{d}}^{\mathrm{s}}(\lambda, \mathbf{v}) \neq \varnothing$ implies $\left(\mathbf{v}, \alpha_{i}\right) \leq 0$.

Proof. Take any point $[B] \in \mathcal{N}_{\mathbf{Q}, \mathbf{d}}^{\mathrm{s}}(\lambda, \mathbf{v})$. Let $\iota: \operatorname{Ker} N_{i} \rightarrow V_{i} \otimes_{\mathbb{C}} R_{d_{i}}$ be the inclusion and $\pi: V_{i} \otimes_{\mathbb{C}} R_{d_{i}} \rightarrow$ Coker $N_{i}$ be the projection. Then Lemma 3.5 together with the assumption $\mathbf{v} \neq \alpha_{i}$ implies that $B_{\leftarrow i} \iota$ is injective and $\pi B_{i \leftarrow}$ is surjective. On the other hand, (3.5) and the assumption for $\lambda_{i}(z)$ imply that

$$
V_{i} \simeq \operatorname{Ker} N_{i} \stackrel{B_{\leftarrow i \iota}}{\longrightarrow} \widehat{V}_{i} \stackrel{\pi B_{i \longleftarrow}}{\longrightarrow} \operatorname{Coker} N_{i} \simeq V_{i}
$$

is a complex. Thus we have

$$
0 \leq \operatorname{dim} \widehat{V}_{i}-2 \operatorname{dim} V_{i}=\sum_{j} a_{i j} d_{j} v_{j}-2 v_{i}
$$

which is equivalent to $\left(\mathbf{v}, \alpha_{i}\right) \leq 0$.

Now applying Crawley-Boevey's argument in [6, Lemma 7.3] to our quiver varieties with multiplicities, we obtain the following:

Proposition 4.8. If $\mathcal{N}_{Q, \mathbf{d}}^{\mathrm{s}}(\lambda, \mathbf{v}) \neq \varnothing$, then $\mathbf{v}$ is a positive root of $\mathfrak{g}(\mathbf{C})$.

Proof. Assume $\mathcal{N}_{\mathbf{Q}, \mathbf{d}}^{\mathrm{s}}(\lambda, \mathbf{v}) \neq \varnothing$ and that $\mathbf{v}$ is not a real root. We show that $\mathbf{v}$ is an imaginary root using [13, Theorem 5.4]; namely, show that there exists $w \in W(\mathbf{C})$ such that $w(\mathbf{v})$ has a connected support and $\left(w(\mathbf{v}), \alpha_{i}\right) \leq 0$ for any $i \in I$.

Assume that there is $i \in I$ such that $\left(\mathbf{v}, \alpha_{i}\right)>0$. The above lemma implies that the top coefficient of $\lambda_{i}(z)$ is nonzero, which together with Theorem 4.2 implies that $\mathcal{N}_{\mathbf{Q}, \mathbf{d}}^{\mathrm{s}}\left(r_{i}(\lambda), s_{i}(\mathbf{v})\right) \neq \varnothing$. In particular we have $s_{i}(\mathbf{v}) \in Q_{+}$, and further $\mathbf{v}-s_{i}(\mathbf{v}) \in \mathbb{Z}_{>0} \alpha_{i}$ by the assumption $\left(\mathbf{v}, \alpha_{i}\right)>0$. We then replace $(\lambda, \mathbf{v})$ with $\left(r_{i}(\lambda), s_{i}(\mathbf{v})\right)$, and repeat this argument. As the components of $\mathbf{v}$ decrease, it eventually stops after finite number of steps, and we finally obtain a pair $(\lambda, \mathbf{v}) \in$ $R^{\mathbf{d}} \times Q_{+}$such that $\left(\mathbf{v}, \alpha_{i}\right) \leq 0$ for all $i \in I$. Additionally, the property $\mathcal{N}_{\mathbf{Q}, \mathbf{d}}^{\mathrm{s}}(\lambda, \mathbf{v}) \neq \varnothing$ clearly implies that the support of $\mathbf{v}$ is connected. The result follows.

\section{Normalization}

In this section we give an application of Boalch's 'shifting trick' to quiver varieties with multiplicities. 


\section{$5.1 \quad$ Shifting trick}

Definition 5.1. Let $(\mathrm{Q}, \mathbf{d})$ be a quiver with multiplicities. A vertex $i \in I$ is called a pole vertex if there exists a unique vertex $j \in I$ such that

$$
d_{j}=1, \quad a_{i k}=a_{k i}=\delta_{j k} \quad \text { for any } k \in I .
$$

The vertex $j$ is called the base vertex for the pole $i$. If furthermore $d_{i}>1$, the pole $i \in I$ is said to be irregular.

Let $i \in I$ be a pole vertex with the base $j \in I$. Then $\widehat{V}_{i}=V_{j} \otimes_{\mathbb{C}} R_{d_{j}}=V_{j}$. In what follows we assume that the top coefficient of $\lambda_{i}(z)$ is nonzero. As the set $\mathcal{N}_{\mathbf{Q}, \mathbf{d}}^{\text {set }}(\lambda, \mathbf{v})$ is empty unless $\operatorname{dim} V_{i} \leq \operatorname{dim} \widehat{V}_{i}=\operatorname{dim} V_{j}$, we also assume $V_{i} \subset V_{j}$ and fix an identification $V_{j} \simeq V_{i} \oplus V_{j} / V_{i}$. Recall the isomorphism given in the previous section:

$$
\mu_{\mathbf{d}, i}^{-1}\left(-\lambda_{i}(z) \operatorname{Id}_{V_{i}}\right) / G_{d_{i}}\left(V_{i}\right) \simeq \mathcal{O} \times \mathbf{M}_{\mathbf{Q}, \mathbf{d}}^{(i)}(\mathbf{V}),
$$

where $\mathcal{O}$ is the $G_{d_{i}}\left(V_{j}\right)$-coadjoint orbit through the element of the form

$$
\Lambda(z)=\left(\begin{array}{cc}
\lambda_{i}(z) \operatorname{Id}_{V_{i}} & 0 \\
0 & 0 \operatorname{Id}_{V_{j} / V_{i}}
\end{array}\right) .
$$

Let us decompose $\Lambda(z)$ as

$$
\Lambda(z)=\Lambda^{0}(z)+z^{-1} \underset{z=0}{\operatorname{res}} \Lambda(z)
$$

according to the decomposition

$$
\mathfrak{g}_{d_{i}}^{*}\left(V_{j}\right)=\mathfrak{b}_{d_{i}}^{*}\left(V_{j}\right) \oplus z^{-1} \mathfrak{g l}\left(V_{j}\right),
$$

where

$$
\mathfrak{b}_{d_{i}}^{*}\left(V_{j}\right):=\operatorname{Ker}\left[\operatorname{res}_{z=0}: \mathfrak{g}_{d_{i}}^{*}\left(V_{j}\right) \rightarrow \mathfrak{g l}\left(V_{j}\right)\right] \simeq z^{-d_{i}} \mathfrak{g l}\left(V_{j}\right)[[z]] / z^{-1} \mathfrak{g l}\left(V_{j}\right)[[z]] .
$$

The above is naturally dual to the Lie algebra $\mathfrak{b}_{d_{i}}\left(V_{j}\right)$ of the unipotent subgroup

$$
B_{d_{i}}\left(V_{j}\right):=\left\{g(z) \in G_{d_{i}}\left(V_{j}\right) \mid g(0)=\operatorname{Id}_{V_{j}}\right\} .
$$

The coadjoint action of $g(z) \in B_{d_{i}}\left(V_{j}\right)$ is given by

$$
(g \cdot \eta)(z)=g(z) \eta(z) g(z)^{-1} \bmod z^{-1} \mathfrak{g l}\left(V_{j}\right)[[z]], \quad \eta(z)=\sum_{k=2}^{d_{j}} \eta_{k} z^{-k} \in \mathfrak{b}_{d_{i}}^{*}\left(V_{j}\right) .
$$

Now consider the $B_{d_{i}}\left(V_{j}\right)$-coadjoint orbit $\check{\mathcal{O}}$ through $\Lambda^{0}(z)$. Let

$$
K:=\mathrm{GL}\left(V_{i}\right) \times \mathrm{GL}\left(V_{j} / V_{i}\right) \subset \mathrm{GL}\left(V_{j}\right)
$$

be the Levi subgroup associated to the decomposition $V_{j}=V_{i} \oplus V_{j} / V_{i}$. The results in this section is based on the following two facts:

Lemma 5.2. The orbit $\check{\mathcal{O}}$ is invariant under the conjugation action by $K$, and there exists a K-equivariant algebraic symplectomorphism

$$
\check{\mathcal{O}} \simeq \operatorname{Hom}\left(V_{j} / V_{i}, V_{i}\right)^{\oplus\left(d_{i}-2\right)} \oplus \operatorname{Hom}\left(V_{i}, V_{j} / V_{i}\right)^{\oplus\left(d_{i}-2\right)}
$$

sending $\Lambda^{0}(z) \in \check{\mathcal{O}}$ to the origin. 
Lemma 5.3. Let $M$ be a holomorphic symplectic manifold with a Hamiltonian action of $\mathrm{GL}\left(V_{j}\right)$ and a moment map $\mu_{M}: M \rightarrow \mathfrak{g l}\left(V_{j}\right)$. Then for any $\zeta \in \mathbb{C}$, the map

$$
\check{\mathcal{O}} \times M \rightarrow \mathfrak{g}_{d_{i}}^{*}\left(V_{j}\right) \times M, \quad(B(z), x) \mapsto\left(B(z)-z^{-1} \mu_{M}(x)-z^{-1} \zeta \operatorname{Id}_{V_{j}}, x\right)
$$

induces a bijection between

(i) the (set-theoretical) symplectic quotient of $\check{\mathcal{O}} \times M$ by the diagonal $K$-action at the level $-\underset{z=0}{\operatorname{res}} \Lambda(z)-\zeta \operatorname{Id}_{V_{j}} ;$ and

(ii) that of $\mathcal{O} \times M$ by the diagonal $\mathrm{GL}\left(V_{j}\right)$-action at the level $-\zeta \operatorname{Id}_{V_{j}}$.

Furthermore, under this bijection a point in the space (i) represents a free $K$-orbit if and only if the corresponding point in the space (ii) represents a free $\mathrm{GL}\left(V_{j}\right)$-orbit, at which the two symplectic forms are intertwined.

Lemma 5.3 is what we call 'Boalch's shifting trick'. We directly check the above two facts in Appendix A.

Remark 5.4. Let $\Lambda^{1}, \Lambda^{2}, \ldots, \Lambda^{k} \in \operatorname{End}(V)$ be mutually commuting endomorphisms of a $\mathbb{C}$ vector space $V$, and suppose that $\Lambda^{2}, \ldots, \Lambda^{k}$ are semisimple. To such endomorphisms we associate

$$
\Lambda(z):=\sum_{j=1}^{k} \Lambda^{j} z^{-j} \in \mathfrak{g}_{k}^{*}(V),
$$

which is called a normal form. Let $\Sigma \subset \mathfrak{g}_{k}^{*}(\mathbb{C})$ be the subset consisting of all residue-free elements $\lambda(z)=\sum_{j=2}^{k} \lambda^{j} z^{-j}$ with $\left(\lambda^{2}, \ldots, \lambda^{k}\right)$ being a simultaneous eigenvalue of $\left(\Lambda^{2}, \ldots, \Lambda^{k}\right)$, and let $V=\bigoplus_{\lambda \in \Sigma} V_{\lambda}$ be the eigenspace decomposition. Then we can express $\Lambda(z)$ as

$$
\Lambda(z)=\bigoplus_{\lambda \in \Sigma}\left(\lambda(z) \operatorname{Id}_{V_{\lambda}}+\frac{\Gamma_{\lambda}}{z}\right), \quad \Gamma_{\lambda}=\left.\Lambda^{1}\right|_{V_{\lambda}} \in \operatorname{End}\left(V_{\lambda}\right) .
$$

It is known that any $A(z) \in \mathfrak{g}_{k}^{*}(V)$ whose leading term is regular semisimple is equivalent to some normal form under the coadjoint action.

Note that $\Lambda(z)$ treated in Lemmas 5.2 and 5.3 is a normal form. A generalization of Lemma 5.2 for an arbitrary normal form has been announced in [4, Appendix C]. Lemma 5.3 is known in the case where $\Lambda(z)$ is a normal form whose leading term is regular semisimple [2]; however, as mentioned in [4], the arguments in [2, Section 2] needed to prove this fact can be generalized to the case where $\Lambda(z)$ is an arbitrary normal form.

We apply Lemma 5.3 to the case where $M=\mathbf{M}_{\mathbf{Q}, \mathbf{d}}^{(i)}(\mathbf{V}), \zeta=\underset{z=0}{\operatorname{res}} \lambda_{j}(z)$. In this case, the symplectic quotient of the space (ii) by the action of $\prod_{k \neq i, j} G_{d_{k}}\left(V_{k}\right)$ turns out to be $\mu_{\mathbf{d}}^{-1}\left(-\lambda \operatorname{Id}_{\mathbf{V}}\right) / G_{\mathbf{d}}(\mathbf{V})$ $=\mathcal{N}_{Q, \mathbf{d}}^{\text {set }}(\lambda, \mathbf{v})$. On the other hand, by Lemma 5.2 , the symplectic quotient of the space (i) by the action of $\prod_{k \neq i, j} G_{d_{k}}\left(V_{k}\right)$ coincides with the symplectic quotient of

$$
\operatorname{Hom}\left(V_{j} / V_{i}, V_{i}\right)^{\oplus\left(d_{i}-2\right)} \oplus \operatorname{Hom}\left(V_{i}, V_{j} / V_{i}\right)^{\oplus\left(d_{i}-2\right)} \oplus \mathbf{M}_{\mathbf{Q}, \mathbf{d}}^{(i)}(\mathbf{V})
$$

by the action of

$$
\mathrm{GL}\left(V_{i}\right) \times \mathrm{GL}\left(V_{j} / V_{i}\right) \times \prod_{k \neq i, j} G_{d_{k}}\left(V_{k}\right),
$$

at the level given by

$$
-\left(\underset{z=0}{\operatorname{res}}\left(\lambda_{i}(z)+\lambda_{j}(z)\right), \underset{z=0}{\operatorname{res}} \lambda_{j}(z),\left(\lambda_{k}(z)\right)_{k \neq i, j}\right) .
$$




\subsection{Normalization}

The observation in the previous subsection leads us to define the following:

Definition 5.5. Let $i \in I$ be an irregular pole vertex of a quiver with multiplicities (Q,d) and $j \in I$ be the base vertex for $i$. Then define $\check{\mathbf{d}}=\left(\check{d}_{k}\right) \in \mathbb{Z}_{>0}^{I}$ by

$\check{d}_{i}:=1, \quad \check{d}_{k}:=d_{k}$ for $k \neq i$,

and let $\check{\mathbf{Q}}=(I, \check{\Omega}, \mathrm{s}, \mathrm{t})$ be the quiver obtained from $(\mathrm{Q}, \mathbf{d})$ as the following:

(i) first, delete a unique arrow joining $i$ and $j$; then

(ii) for each arrow $h$ with $\mathrm{t}(h)=j$, draw an arrow from $\mathrm{s}(h)$ to $i$;

(iii) for each arrow $h$ with $\mathrm{s}(h)=j$, draw an arrow from $i$ to $\mathrm{t}(h)$;

(iv) finally, draw $d_{i}-2$ arrows from $j$ to $i$.

The transformation $(\mathbf{Q}, \mathbf{d}) \mapsto(\check{\mathbf{Q}}, \mathbf{\mathbf { d }})$ is called the normalization at $i$.

The adjacency matrix $\check{\mathbf{A}}=\left(\check{a}_{k l}\right)$ of the underlying graph of $\check{\mathbf{Q}}$ satisfies

$$
\check{a}_{k l}=\check{a}_{l k}= \begin{cases}d_{i}-2 & \text { if }(k, l)=(i, j), \\ a_{j l} & \text { if } k=i, l \neq j, \\ a_{k l} & \text { if } k, l \neq i\end{cases}
$$

Example 5.6. (i) Suppose that $(\mathrm{Q}, \mathbf{d})$ has the graph with multiplicities given below

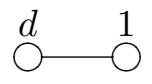

Here we assume $d>1$. The left vertex is an irregular pole, at which we can perform the normalization and the resulting $(\check{Q}, \check{\mathbf{d}})$ has the underlying graph with multiplicities drawn below

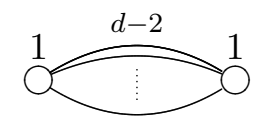

The number of edges joining the two vertices are $d-2$. If $d=3$, the Kac-Moody algebra associated to $(\mathbf{Q}, \mathbf{d})$ is of type $G_{2}$, while the one associated to $(\check{\mathbf{Q}}, \check{\mathbf{d}})$ is of type $A_{2}$. If $d=4$, the Kac-Moody algebra associated to $(\mathrm{Q}, \mathbf{d})$ is of type $A_{2}^{(2)}$, while the one associated to $(\check{\mathbf{Q}}, \check{\mathbf{d}})$ is of type $A_{1}^{(1)}$.

(ii) Suppose that $(\mathrm{Q}, \mathbf{d})$ has the graph with multiplicities given below

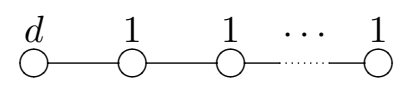

Here we assume $d>1$ and the number of vertices is $n \geq 3$. The vertex on the far left is an irregular pole, at which we can perform the normalization and the resulting ( $\check{Q}, \check{\mathbf{d}})$ has the underlying graph with multiplicities drawn below

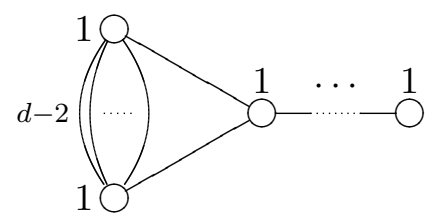


If $d=2$, then the Kac-Moody algebra associated to $(\mathbf{Q}, \mathbf{d})$ is of type $C_{n}$, while the one associated to $(\check{\mathbf{Q}}, \breve{\mathbf{d}})$ is of type $A_{3}$ if $n=3$ and of type $D_{n}$ if $n>3$. If $(d, n)=(3,3)$, the Kac-Moody algebra associated to $(\mathbf{Q}, \mathbf{d})$ is of type $D_{4}^{(3)}$, while the one associated to $(\check{\mathbf{Q}}, \check{\mathbf{d}})$ is of type $A_{2}^{(1)}$.

(iii) Suppose that $(\mathbf{Q}, \mathbf{d})$ has the graph with multiplicities given below

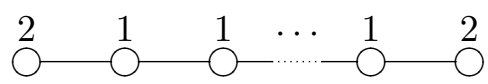

Here the number of vertices is $n \geq 3$. The associated Kac-Moody algebra is of type $C_{n-1}^{(1)}$. It has two irregular poles. Let us perform the normalization at the vertex on the far right. If $n=3$, the resulting $(\check{Q}, \breve{\mathbf{d}})$ has the underlying graph with multiplicities drawn below

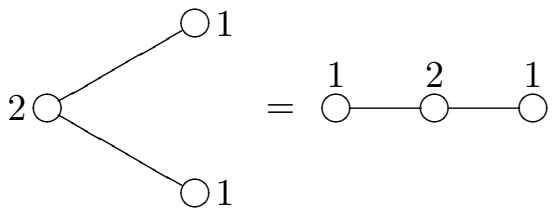

The associated Kac-Moody algebra is of type $D_{3}^{(2)}$. If $n \geq 4$, the resulting $(\check{\mathbf{Q}}, \check{\mathbf{d}})$ has the underlying graph with multiplicities drawn below

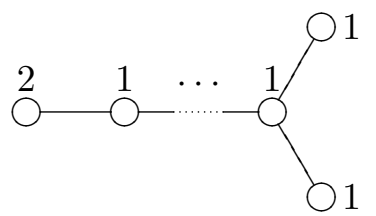

The associated Kac-Moody algebra is of type $A_{2 n-3}^{(2)}$. The vertex on the far left is still an irregular pole, at which we can perform the normalization again. If $n=4$, the resulting $(\check{\mathbf{Q}}, \check{\mathbf{d}})$ has the underlying graph with multiplicities drawn below

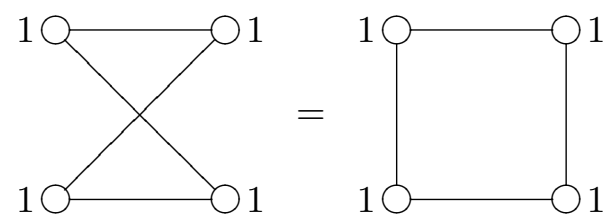

The associated Kac-Moody algebra is of type $A_{3}^{(1)}$. If $n>4$, the resulting ( $\left.\check{\mathbf{Q}}, \check{\mathbf{d}}\right)$ has the underlying graph with multiplicities drawn below

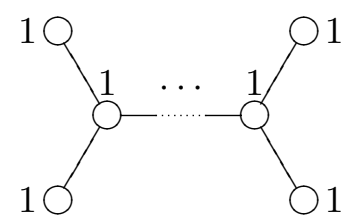

The associated Kac-Moody algebra is of type $D_{n-1}^{(1)}$.

In the situation discussed in the previous subsection, let $\check{\mathbf{V}}=\bigoplus_{k} \check{V}_{k}$ be the $I$-graded vector space defined by

$$
\check{V}_{j}:=V_{j} / V_{i}, \quad \check{V}_{k}:=V_{k} \text { for } k \neq j .
$$

Then we see that the group in (5.2) coincides with $G_{\check{\mathbf{d}}}(\check{\mathbf{V}})$. Furthermore, the following holds: 
Lemma 5.7. The symplectic vector space in (5.1) coincides with $\mathbf{M}_{\check{Q}, \check{\mathbf{d}}}(\check{\mathbf{V}})$.

Proof. The definitions of $\check{Q}, \check{\mathbf{d}}, \check{\mathbf{V}}$ imply

$$
\begin{aligned}
& \operatorname{Rep}_{\breve{Q}}\left(\check{\mathbf{V}}_{\check{\mathbf{d}}}\right)=\bigoplus_{\substack{h \in \check{\Omega} \\
h: j \rightarrow i}} \operatorname{Hom}\left(V_{j} / V_{i}, V_{i}\right) \\
& \oplus \bigoplus_{k \neq i, j}\left(\bigoplus_{\substack{h \in \check{\Omega} \\
h: k \rightarrow i}} \operatorname{Hom}\left(V_{k} \otimes_{\mathbb{C}} R_{d_{k}}, V_{i}\right) \oplus \bigoplus_{\substack{h \in \check{\Omega} \\
h: i \rightarrow k}} \operatorname{Hom}\left(V_{i}, V_{k} \otimes_{\mathbb{C}} R_{d_{k}}\right)\right) \\
& \oplus \bigoplus_{k \neq i, j}\left(\bigoplus_{\substack{h \in \check{\Omega} \\
h: k \rightarrow j}} \operatorname{Hom}\left(V_{k} \otimes_{\mathbb{C}} R_{d_{k}}, V_{j} / V_{i}\right) \oplus \bigoplus_{\substack{h \in \check{\Omega} \\
h: j \rightarrow k}} \operatorname{Hom}\left(V_{j} / V_{i}, V_{k} \otimes_{\mathbb{C}} R_{d_{k}}\right)\right) \\
& \oplus \bigoplus_{k, l \neq i, j} \bigoplus_{\substack{h \in \check{\Omega} \\
h: k \rightarrow l}} \operatorname{Hom}\left(V_{k} \otimes_{\mathbb{C}} R_{d_{k}}, V_{l} \otimes_{\mathbb{C}} R_{d_{l}}\right) \\
& =\operatorname{Hom}\left(V_{j} / V_{i}, V_{i}\right)^{\oplus\left(d_{i}-2\right)} \\
& \oplus \bigoplus_{k \neq i, j}\left(\bigoplus_{\substack{h \in \Omega \\
h: k \rightarrow j}} \operatorname{Hom}\left(V_{k} \otimes_{\mathbb{C}} R_{d_{k}}, V_{j}\right) \oplus \bigoplus_{\substack{h \in \Omega \\
h: j \rightarrow k}} \operatorname{Hom}\left(V_{j}, V_{k} \otimes_{\mathbb{C}} R_{d_{k}}\right)\right) \\
& \oplus \bigoplus_{k, l \neq i, j} \bigoplus_{\substack{h \in \Omega \\
h: k \rightarrow l}} \operatorname{Hom}\left(V_{k} \otimes_{\mathbb{C}} R_{d_{k}}, V_{l} \otimes_{\mathbb{C}} R_{d_{l}}\right) \\
& =\operatorname{Hom}\left(V_{j} / V_{i}, V_{i}\right)^{\oplus\left(d_{i}-2\right)} \oplus \bigoplus_{\substack{h \in \Omega \\
\mathrm{s}(h) \mathrm{t}(h) \neq i}} \operatorname{Hom}\left(V_{\mathrm{s}(h)} \otimes_{\mathbb{C}} R_{d_{\mathrm{s}(h)}}, V_{\mathrm{t}(h)} \otimes_{\mathbb{C}} R_{d_{\mathrm{t}(h)}}\right) .
\end{aligned}
$$

Taking the cotangent bundle, we thus see that $\mathbf{M}_{\check{Q}, \check{\mathbf{d}}}(\check{\mathbf{V}})$ coincides with (5.1).

Set $\check{\mathbf{v}}:=\operatorname{dim} \check{\mathbf{V}}$ and

$$
\check{\lambda}=\left(\check{\lambda}_{k}(z)\right) \in R^{\check{\mathbf{d}}}, \quad \check{\lambda}_{k}(z):= \begin{cases}z^{-1} \underset{z=0}{\operatorname{res}}\left(\lambda_{i}(z)+\lambda_{j}(z)\right) & \text { if } k=i, \\ z^{-1} \underset{z=0}{\operatorname{res}} \lambda_{j}(z) & \text { if } k=j, \\ \lambda_{k}(z) & \text { if } k \neq i, j .\end{cases}
$$

Then the value given in $(5.3)$ coincides with $-\check{\lambda}$. Note that

$$
\check{\mathbf{v}} \cdot \operatorname{res} \check{\lambda}=v_{i} \underset{z=0}{\operatorname{res}}\left(\lambda_{i}(z)+\lambda_{j}(z)\right)+\left(v_{j}-v_{i}\right) \underset{z=0}{\operatorname{res}} \lambda_{j}(z)+\sum_{k \neq i, j} v_{k} \underset{z=0}{\operatorname{res}} \lambda_{k}(z)=\mathbf{v} \cdot \operatorname{res} \lambda .
$$

Now we state the main result of this section.

Theorem 5.8. Let $i \in I$ be an irregular pole vertex of a quiver with multiplicities (Q,d) and $j \in I$ be the base vertex for $i$. Let $(\check{\mathbf{Q}}, \check{\mathbf{d}})$ be the quiver with multiplicities obtained by the normalization of $(\mathbf{Q}, \mathbf{d})$ at $i$. Take $(\lambda, \mathbf{v}) \in R^{\mathbf{d}} \times Q_{+}$such that the top coefficient $\lambda_{i, d_{i}}$ of $\lambda_{i}(z)$ is nonzero. Then the quiver varieties $\mathcal{N}_{\mathbf{Q}, \mathbf{d}}^{\mathrm{s}}(\lambda, \mathbf{v})$ and $\mathcal{N}_{\breve{Q}, \check{\mathbf{d}}}^{\mathrm{s}}(\check{\lambda}, \check{\mathbf{v}})$ are symplectomorphic to each other.

Proof. We have already constructed a bijection between $\mathcal{N}_{Q, \mathbf{d}}^{\text {set }}(\lambda, \mathbf{v})$ and $\mathcal{N}_{\check{Q}, \check{\mathbf{d}}}^{\text {set }}(\check{\lambda}, \check{\mathbf{v}})$. Thanks to Lemma 5.3, in order to prove the assertion it is sufficient to check that the bijection maps $\mathcal{N}_{\mathbf{Q}, \mathbf{d}}^{\mathrm{s}}(\lambda, \mathbf{v})$ onto $\mathcal{N}_{\check{\mathbf{Q}, \breve{\mathbf{d}}}}^{\mathrm{s}}(\check{\lambda}, \check{\mathbf{v}})$. It immediately follows from the three lemmas below. 
Lemma 5.9. A point $B \in \mu_{\mathbf{d}}^{-1}\left(-\lambda \mathrm{Id}_{\mathbf{v}}\right)$ is stable if and only if the corresponding $\left(A(z), B_{\neq i}\right)=$ $\left(\sum_{l=1}^{d_{i}} A_{l} z^{-l}, B_{\neq i}\right) \in \mathcal{O} \times \mathbf{M}_{\mathbf{Q}, \mathbf{d}}^{(i)}(\mathbf{V})$ satisfies the following condition: if a collection of subspaces $S_{k} \subset V_{k} \otimes_{\mathbb{C}} R_{d_{k}}, k \neq i$ satisfies

$$
\begin{array}{ll}
N_{k}\left(S_{k}\right) \subset S_{k} & \text { for } k \neq i, j ; \\
B_{h}\left(S_{\mathrm{s}(h)}\right) \subset S_{\mathrm{t}(h)} & \text { for } h \in H \text { with } \mathrm{t}(h), \mathrm{s}(h) \neq i ; \\
A_{l}\left(S_{j}\right) \subset S_{j} & \text { for } l=1, \ldots, d_{i},
\end{array}
$$

then $S_{k}=0(k \neq i)$ or $S_{k}=V_{k} \otimes_{\mathbb{C}} R_{d_{k}}(k \neq i)$.

Proof. This is similar to Lemma 4.4. First, assume that $B$ is stable and that a collection of subspaces $S_{k} \subset V_{k} \otimes_{\mathbb{C}} R_{d_{k}}, k \neq i$ satisfies (5.5). We define

$$
S_{i}:=\sum_{l=1}^{d_{i}} N_{i}^{l-1} B_{i \leftarrow}\left(S_{j}\right),
$$

and set $\mathbf{S}:=\bigoplus_{k \in I} S_{k} \subset \mathbf{V}_{\mathbf{d}}$. Then $N_{i}\left(S_{i}\right) \subset S_{i}, B_{i \leftarrow}\left(S_{j}\right) \subset S_{i}$ and

$$
B_{\leftarrow i}\left(S_{i}\right)=\sum_{l} B_{\leftarrow i} N_{i}^{l-1} B_{i \leftarrow}\left(S_{j}\right)=\sum_{l} A_{l}\left(S_{j}\right) \subset S_{j}
$$

imply that $\mathbf{S}$ is $B$-invariant. Since $B$ is stable, we thus have $\mathbf{S}=0$ or $\mathbf{S}=\mathbf{V}_{\mathbf{d}}$.

Next assume that the pair $\left(A(z), B_{\neq i}\right)$ satisfies the condition in the statement. Let $\mathbf{S}=\bigoplus_{k} S_{k}$ be a $B$-invariant subspace of $\mathbf{V}_{\mathbf{d}}$ satisfying $N_{k}\left(S_{k}\right) \subset S_{k}$ for all $k \in I$. Then clearly the collection $S_{k}, k \neq i$ satisfies (5.5), and hence $S_{k}=0(k \neq i)$ or $S_{k}=V_{k} \otimes_{\mathbb{C}} R_{d_{k}}(k \neq i)$. If $S_{k}=0, k \neq i$, we have $B_{\leftarrow i}\left(S_{i}\right)=0$, which implies $S_{i}=0$ since $\operatorname{Ker} B_{\leftarrow i} \cap \operatorname{Ker} N_{i}=0$ by Lemma 3.5 and $\left.N_{i}\right|_{S_{i}}$ is nilpotent. Dualizing the argument, we easily see that $S_{i}=V_{i} \otimes_{\mathbb{C}} R_{d_{i}}$ if $S_{k}=V_{k} \otimes_{\mathbb{C}} R_{d_{k}}, k \neq i$.

Lemma 5.10. A point $B^{\prime} \in \mu_{\breve{\mathbf{d}}}^{-1}\left(-\check{\lambda} \mathrm{Id}_{\check{\mathbf{v}}}\right)$ is stable if and only if the corresponding $\left(A^{0}(z), B_{\neq i}\right)=$ $\left(\sum_{l=2}^{d_{i}} A_{l}^{0} z^{-l}, B_{\neq i}\right) \in \check{\mathcal{O}} \times \mathbf{M}_{\mathbf{Q}, \mathbf{d}}^{(i)}(\mathbf{V})$ satisfies the following condition: if an I-graded subspace $\mathbf{S}=\bigoplus_{k} S_{k}$ of $\check{\mathbf{V}}_{\check{\mathbf{d}}}=\check{\mathbf{V}} \otimes_{\mathbb{C}} R_{\check{\mathbf{d}}}$ satisfies

$$
\begin{array}{ll}
N_{k}\left(S_{k}\right) \subset S_{k} & \text { for } k \neq i, j ; \\
B_{h}\left(S_{\mathrm{s}(h)}\right) \subset S_{\mathrm{t}(h)} & \text { for } h \in H \text { with }(\mathrm{t}(h), \mathrm{s}(h)) \neq(i, j),(j, i) ; \\
A_{l}^{0}\left(S_{i} \oplus S_{j}\right) \subset S_{i} \oplus S_{j} & \text { for } l=2, \ldots, d_{i},
\end{array}
$$

then $\mathbf{S}=0$ or $\mathbf{S}=\check{\mathbf{V}}_{\check{\mathbf{d}}}$.

Proof. In Appendix A, we show that all the block components of $A_{l}^{0}$ relative to the decomposition $V_{j}=\check{V}_{i} \oplus \check{V}_{j}$ are described as a (non-commutative) polynomial in $B_{h}^{\prime}$ over $h \in H$ with $(\mathrm{t}(h), \mathrm{s}(h))=(i, j)$ or $(j, i)$, and vice versa (see Remark A.3, where $A^{0}$ is denoted by $B$ and $B_{h}^{\prime}$ for such $h$ are denoted by $a_{k}^{\prime}, b_{k}^{\prime}$ ). Hence an $I$-graded subspace $\mathbf{S}$ of $\check{\mathbf{V}}_{\check{\mathbf{d}}}$ satisfies (5.6) if and only if it is $B^{\prime}$-invariant and $N_{k}\left(S_{k}\right) \subset S_{k}$ for $k \neq i, j$.

Lemma 5.11. Let $\left(A^{0}(z), B_{\neq i}\right) \in \check{\mathcal{O}} \times \mathbf{M}_{\mathbf{Q}, \mathbf{d}}^{(i)}(\mathbf{V})$ and let $\left(A(z), B_{\neq i}\right) \in \mathcal{O} \times \mathbf{M}_{\mathbf{Q}, \mathbf{d}}^{(i)}(\mathbf{V})$ be the corresponding pair under the map given in Lemma 5.3. Then $\left(A^{0}(z), B_{\neq i}\right)$ satisfies the condition in Lemma 5.10 if and only if $\left(A(z), B_{\neq i}\right)$ satisfies the one in Lemma 5.9. 
Proof. By definition we have

$$
A(z)=A^{0}(z)-z^{-1} \sum_{\substack{\mathrm{t}(h)=j, \mathrm{~s}(h) \neq i}} \epsilon(h) B_{h} B_{\bar{h}}-\lambda_{j}(z) \operatorname{Id}_{V_{j}},
$$

so the 'if' part is clear. To prove the 'only if' part, note that if a collection of subspaces $S_{k} \subset V_{k} \otimes_{\mathbb{C}} R_{d_{k}}, k \neq i$ satisfies (5.5), then in particular $S_{j}$ is preserved by the action of

$$
A_{d_{i}}=A_{d_{i}}^{0}=\lambda_{i, d_{i}} \operatorname{Id}_{\check{V}_{i}} \oplus 0 \operatorname{Id}_{\check{V}_{j}},
$$

and hence is homogeneous relative to the decomposition $V_{j}=\check{V}_{i} \oplus \check{V}_{j}$;

$$
S_{j}=\left(S_{j} \cap \check{V}_{i}\right) \oplus\left(S_{j} \cap \check{V}_{j}\right) .
$$

Now the result immediately follows.

\section{$5.3 \quad$ Weyl groups}

Let $(\mathrm{Q}, \mathbf{d})$ be a quiver with multiplicities having an irregular pole vertex $i \in I$ with base $j \in I$, and let $(\check{\mathbf{Q}}, \check{\mathbf{d}})$ be the one obtained by the normalization of $(\mathbf{Q}, \mathbf{d})$ at $i$. In this subsection we discuss on the relation between the two Weyl groups associated to $(\mathbf{Q}, \mathbf{d})$ and $(\check{Q}, \check{\mathbf{d}})$.

Recall our notation for objects relating to the Kac-Moody algebra; $\mathbf{C}=2 \mathrm{Id}-\mathbf{A D}$ is the generalized Cartan matrix associated to $(\mathbf{Q}, \mathbf{d})$, and $\mathfrak{h}, Q, \alpha_{k}, s_{k}$, the Cartan subalgebra, the root lattice, the simple roots, and the simple reflections, of the corresponding Kac-Moody algebra $\mathfrak{g}(\mathbf{C})$. In what follows we denote by $\check{\mathbf{C}}, \check{\mathbf{D}}, \check{\mathfrak{h}}, \check{Q}, \check{\alpha}_{k}, \check{s}_{k}$, the similar objects associated to $(\check{\mathbf{Q}}, \check{\mathbf{d}})$.

Let $\varphi: Q \rightarrow \check{Q}$ be the linear map defined by $\mathbf{v} \mapsto \check{\mathbf{v}}=\mathbf{v}-v_{i} \check{\alpha}_{j}$. The same letter is also used on the matrix representing $\varphi$ with respect to the simple roots.

Lemma 5.12. The identity ${ }^{t} \varphi \check{\mathbf{D}} \check{\mathbf{C}} \varphi=\mathbf{D C}$ holds.

Proof. To prove it, we express the matrices in block form with respect to the decomposition of the index set $I=\{i\} \sqcup\{j\} \sqcup(I \backslash\{i, j\})$. First, $\varphi$ is expressed as

$$
\varphi=\left(\begin{array}{ccc}
1 & 0 & 0 \\
-1 & 1 & 0 \\
0 & 0 & \text { Id }
\end{array}\right)
$$

By the properties of $i$ and $j$, the matrices $\mathbf{D}$ and $\mathbf{A}$ are respectively expressed as

$$
\mathbf{D}=\left(\begin{array}{ccc}
d_{i} & 0 & 0 \\
0 & 1 & 0 \\
0 & 0 & \mathbf{D}^{\prime}
\end{array}\right), \quad \mathbf{A}=\left(\begin{array}{ccc}
0 & 1 & 0 \\
1 & 0 & { }^{t} \mathbf{a} \\
0 & \mathbf{a} & \mathbf{A}^{\prime}
\end{array}\right)
$$

where $\mathbf{D}^{\prime}$ (resp. $\mathbf{A}^{\prime}$ ) is the sub-matrix of $\mathbf{D}$ (resp. A) obtained by restricting the index set to $I \backslash\{i, j\}$, and $\mathbf{a}=\left(a_{k j}\right)_{k \neq i, j}$. By the definition of the normalization, the matrices $\check{\mathbf{D}}$ and $\check{\mathbf{A}}$ are then respectively expressed as

$$
\check{\mathbf{D}}=\left(\begin{array}{ccc}
1 & 0 & 0 \\
0 & 1 & 0 \\
0 & 0 & \mathbf{D}^{\prime}
\end{array}\right), \quad \check{\mathbf{A}}=\left(\begin{array}{ccc}
0 & d_{i}-2 & { }^{t} \mathbf{a} \\
d_{i}-2 & 0 & { }^{t} \mathbf{a} \\
\mathbf{a} & \mathbf{a} & \mathbf{A}^{\prime}
\end{array}\right) .
$$

Now we check the identity. We have

$$
\mathbf{D C}=2 \mathbf{D}-\mathbf{D A D}=\left(\begin{array}{ccc}
2 d_{i} & 0 & 0 \\
0 & 2 & 0 \\
0 & 0 & 2 \mathbf{D}^{\prime}
\end{array}\right)-\left(\begin{array}{ccc}
0 & d_{i} & 0 \\
d_{i} & 0 & { }^{t} \mathbf{a D}^{\prime} \\
0 & \mathbf{D}^{\prime} \mathbf{a} & \mathbf{D}^{\prime} \mathbf{A}^{\prime} \mathbf{D}^{\prime}
\end{array}\right)
$$




$$
=\left(\begin{array}{ccc}
2 d_{i} & -d_{i} & 0 \\
-d_{i} & 2 & -{ }^{t} \mathbf{a D}^{\prime} \\
0 & -\mathbf{D}^{\prime} \mathbf{a} & 2 \mathrm{Id}-\mathbf{D}^{\prime} \mathbf{A}^{\prime} \mathbf{D}^{\prime}
\end{array}\right) .
$$

On the other hand,

$$
\begin{aligned}
\check{\mathbf{D}} \check{\mathbf{C}}=2 \check{\mathbf{D}}-\check{\mathbf{D}} \check{\mathbf{A}} \check{\mathbf{D}} & =\left(\begin{array}{ccc}
2 & 0 & 0 \\
0 & 2 & 0 \\
0 & 0 & 2 \mathbf{D}^{\prime}
\end{array}\right)-\left(\begin{array}{ccc}
0 & d_{i}-2 & { }^{t} \mathbf{a D}^{\prime} \\
d_{i}-2 & 0 & { }^{t} \mathbf{a D}^{\prime} \\
\mathbf{D}^{\prime} \mathbf{a} & \mathbf{D}^{\prime} \mathbf{a} & \mathbf{D}^{\prime} \mathbf{A}^{\prime} \mathbf{D}^{\prime}
\end{array}\right) \\
& =\left(\begin{array}{ccc}
2 & 2-d_{i} & -{ }^{t} \mathbf{a} \mathbf{D}^{\prime} \\
2-d_{i} & 2 & -{ }^{t} \mathbf{a D}^{\prime} \\
-\mathbf{D}^{\prime} \mathbf{a} & -\mathbf{D}^{\prime} \mathbf{a} & 2 \mathrm{Id}-\mathbf{D}^{\prime} \mathbf{A}^{\prime} \mathbf{D}^{\prime}
\end{array}\right) .
\end{aligned}
$$

Hence

$$
\begin{aligned}
{ }^{t} \varphi \check{\mathbf{D}} \check{\mathbf{C}} \varphi & =\left(\begin{array}{ccc}
1 & -1 & 0 \\
0 & 1 & 0 \\
0 & 0 & \mathrm{Id}
\end{array}\right)\left(\begin{array}{ccc}
2 & 2-d_{i} & -{ }^{t} \mathbf{a} \mathbf{D}^{\prime} \\
2-d_{i} & 2 & -{ }^{t} \mathbf{a D}^{\prime} \\
-\mathbf{D}^{\prime} \mathbf{a} & -\mathbf{D}^{\prime} \mathbf{a} & 2 \mathrm{Id}-\mathbf{D}^{\prime} \mathbf{A}^{\prime} \mathbf{D}^{\prime}
\end{array}\right)\left(\begin{array}{ccc}
1 & 0 & 0 \\
-1 & 1 & 0 \\
0 & 0 & \mathrm{Id}
\end{array}\right) \\
& =\left(\begin{array}{ccc}
d_{i} & -d_{i} & 0 \\
2-d_{i} & 2 & -{ }^{t} \mathbf{a} \mathbf{D}^{\prime} \\
-\mathbf{D}^{\prime} \mathbf{a} & -\mathbf{D}^{\prime} \mathbf{a} & 2 \mathrm{Id}-\mathbf{D}^{\prime} \mathbf{A}^{\prime} \mathbf{D}^{\prime}
\end{array}\right)\left(\begin{array}{ccc}
1 & 0 & 0 \\
-1 & 1 & 0 \\
0 & 0 & \mathrm{Id}
\end{array}\right) \\
& =\left(\begin{array}{ccc}
2 d_{i} & -d_{i} & 0 \\
-d_{i} & 2 & -{ }^{t} \mathbf{a D}^{\prime} \\
0 & -\mathbf{D}^{\prime} \mathbf{a} & 2 \mathrm{Id}-\mathbf{D}^{\prime} \mathbf{A}^{\prime} \mathbf{D}^{\prime}
\end{array}\right)=\mathbf{D C} .
\end{aligned}
$$

The above lemma implies that the map $\varphi$ preserves the inner product. Furthermore it also implies $\operatorname{rank} \check{\mathbf{C}}=\operatorname{rank} \mathbf{C}$, which means $\operatorname{dim} \mathfrak{h}=\operatorname{dim} \check{\mathfrak{h}}$. Thus we can extend $\varphi$ to an isomorphism $\tilde{\varphi}: \mathfrak{h}^{*} \rightarrow \check{\mathfrak{h}}^{*}$ preserving the inner product.

Note that by the definition of normalization, the permutation of the indices $i$ and $j$, which we denote by $\sigma$, has no effect on the matrix $\check{\mathbf{C}}$. Hence it defines an involution of $W(\check{\mathbf{C}})$, or equivalently, a homomorphism $\mathbb{Z} / 2 \mathbb{Z} \rightarrow \operatorname{Aut}(W(\check{\mathbf{C}}))$.

Proposition 5.13. Under the isomorphism $\tilde{\varphi}$, the Weyl group $W(\mathbf{C})$ associated to $\mathbf{C}$ is isomorphic to the semidirect product $W(\check{\mathbf{C}}) \rtimes \mathbb{Z} / 2 \mathbb{Z}$ of the one associated to $\check{\mathbf{C}}$ and $\mathbb{Z} / 2 \mathbb{Z}$ by the permutation $\sigma$.

Proof. By the construction of $\tilde{\varphi}$ we have

$$
\tilde{\varphi}\left(\alpha_{k}\right)= \begin{cases}\check{\alpha}_{i}-\check{\alpha}_{j} & \text { if } k=i, \\ \check{\alpha}_{k} & \text { if } k \neq i .\end{cases}
$$

As $\tilde{\varphi}$ preserves the inner product, the above implies that for $k \neq i$, the map $\tilde{\varphi} s_{k} \tilde{\varphi}^{-1}$ coincides with the reflection $\check{s}_{k}$ relative to $\tilde{\varphi}\left(\alpha_{k}\right)=\check{\alpha}_{k}$, and the map $\tilde{\varphi} s_{i} \tilde{\varphi}^{-1}$ coincides with the reflection relative to $\tilde{\varphi}\left(\alpha_{i}\right)=\check{\alpha}_{i}-\check{\alpha}_{j}$. Note that since the matrix $\check{\mathbf{D}} \check{\mathbf{C}}$ is invariant under the permutation $\sigma$, we have

$$
\left(\check{\alpha}_{i}+\check{\alpha}_{j}, \check{\alpha}_{i}-\check{\alpha}_{j}\right)=0, \quad\left(\check{\alpha}_{k}, \check{\alpha}_{i}-\check{\alpha}_{j}\right)=0, \quad k \neq i, j,
$$

which imply that $\tilde{\varphi} s_{i} \tilde{\varphi}^{-1}\left(\check{\alpha}_{k}\right)=\check{\alpha}_{\sigma(k)}$ for any $k \in I$. Hence the map $\left(\tilde{\varphi} s_{i} \tilde{\varphi}^{-1}\right) \check{s}_{k}\left(\tilde{\varphi} s_{i} \tilde{\varphi}^{-1}\right)^{-1}$, which is the reflection relative to $\tilde{\varphi} s_{i} \tilde{\varphi}^{-1}\left(\check{\alpha}_{k}\right)$, coincides with $\check{s}_{\sigma(k)}$ for each $k$. Now the result immediately follows. 
We can easily check that

$$
\operatorname{res}(\check{\lambda})={ }^{t} \varphi^{-1}(\operatorname{res}(\lambda)) \quad \text { for } \lambda \in R^{\mathbf{d}} .
$$

Note that the action of $W(\check{\mathbf{C}})$ on $R^{\check{\mathbf{d}}}$ naturally extends to an action of $W(\check{\mathbf{C}}) \rtimes \mathbb{Z} / 2 \mathbb{Z}$. We see from the above relation that the map $R^{\mathbf{d}} \rightarrow R^{\check{\mathbf{d}}}, \lambda \mapsto \check{\lambda}$ is equivariant, and hence so is the map $R^{\mathbf{d}} \times Q \rightarrow R^{\check{\mathbf{d}}} \times \check{Q},(\lambda, \mathbf{v}) \mapsto(\check{\lambda}, \check{\mathbf{v}})$, with respect to the isomorphism $W(\mathbf{C}) \simeq W(\check{\mathbf{C}}) \rtimes \mathbb{Z} / 2 \mathbb{Z}$ given in Proposition 5.13.

\section{Naive moduli of meromorphic connections on $\mathbb{P}^{1}$}

This final section is devoted to study moduli spaces of meromorphic connections on the trivial bundle over $\mathbb{P}^{1}$ with some particular type of singularities.

\subsection{Naive moduli}

When constructing the moduli spaces of meromorphic connections, one usually fix the 'formal type' of singularities. However, we fix here the 'truncated formal type', and consider the corresponding 'naive' moduli space. Actually in generic case, such a naive moduli space gives the moduli space in the usual sense, which will be explained in Remark 6.5.

Fix $n \in \mathbb{Z}_{>0}$ and

- a nonzero finite-dimensional $\mathbb{C}$-vector space $V$;

- positive integers $k_{1}, k_{2}, \ldots, k_{n}$;

- mutually distinct points $t_{1}, t_{2}, \ldots, t_{n}$ in $\mathbb{C}$.

Then consider a system

$$
\frac{\mathrm{d} u}{\mathrm{~d} z}=A(z) u(z), \quad A(z)=\sum_{i=1}^{n} \sum_{j=1}^{k_{i}} \frac{A_{i, j}}{\left(z-t_{i}\right)^{j}}, \quad A_{i, j} \in \operatorname{End}(V)
$$

of linear ordinary differential equations with rational coefficients. It has a pole at $t_{i}$ of order at most $k_{i}$ for each $i$, and (possibly) a simple pole at $\infty$ with residue $-\sum_{i} A_{i, 1}$. We identify such a system with its coefficient matrix $A(z)$, which may be regarded as an element of $\bigoplus_{i} \mathfrak{g}_{k_{i}}^{*}(V)$ via $A(z) \mapsto\left(A_{i}\right), A_{i}(z):=\sum_{k} A_{i, j} z^{-j}$.

After Boalch [2], we introduce the following (the terminologies used here are different from his):

Definition 6.1. For a system $A(z)=\left(A_{i}\right) \in \bigoplus_{i} \mathfrak{g}_{k_{i}}^{*}(V)$ and each $i=1, \ldots, n$, the $G_{k_{i}}(V)$ coadjoint orbit through $A_{i}$ is called the truncated formal type of $A(z)$ at $t_{i}$.

For given coadjoint orbits $\mathcal{O}_{i} \subset \mathfrak{g}_{k_{i}}^{*}(V), i=1, \ldots, n$, the set

$$
\mathcal{M}^{\text {set }}\left(\mathcal{O}_{1}, \ldots, \mathcal{O}_{n}\right):=\left\{A(z) \in \prod_{i=1}^{n} \mathcal{O}_{i} \mid \sum_{i=1}^{n} \operatorname{res}_{z=t_{i}} A(z)=0\right\} / \operatorname{GL}(V)
$$

is called the naive moduli space of systems having a pole of truncated formal type $\mathcal{O}_{i}$ at each $t_{i}$, $i=1, \ldots, n$.

Note that $\prod_{i} \mathcal{O}_{i}$ is a holomorphic symplectic manifold, and the map

$$
\prod_{i=1}^{n} \mathcal{O}_{i} \rightarrow \bigoplus_{i=1}^{n} \mathfrak{g}_{k_{i}}^{*}(V), \quad A(z) \mapsto \sum_{i=1}^{n} \operatorname{res}_{z=t_{i}} A(z)
$$


is a moment map with respect to the simultaneous $\mathrm{GL}(V)$-conjugation action. Hence the set $\mathcal{M}^{\text {set }}\left(\mathcal{O}_{1}, \ldots, \mathcal{O}_{n}\right)$ is a set-theoretical symplectic quotient.

It is also useful to introduce the following $\zeta$-twisted naive moduli space:

$$
\mathcal{M}_{\zeta}^{\mathrm{set}}\left(\mathcal{O}_{1}, \ldots, \mathcal{O}_{n}\right):=\left\{A(z) \in \prod_{i=1}^{n} \mathcal{O}_{i} \mid \sum_{i=1}^{n} \operatorname{res}_{z=t_{i}} A(z)=-\zeta \operatorname{Id}_{V}\right\} / \operatorname{GL}(V) \quad(\zeta \in \mathbb{C}) .
$$

Definition 6.2. A system $A(z) \in \bigoplus_{i} \mathfrak{g}_{k_{i}}^{*}(V)$ is said to be irreducible if there is no nonzero proper subspace $S \subset V$ preserved by all the coefficient matrices $A_{i, j}$.

If $A(z) \in \bigoplus_{i} \mathfrak{g}_{k_{i}}^{*}(V)$ is irreducible, Schur's lemma shows that the stabilizer of $A(z)$ with respect to the $\mathrm{GL}(V)$-action is equal to $\mathbb{C}^{\times}$, and furthermore one can show that the action on the set of irreducible systems in $\prod_{i} \mathcal{O}_{i}$ is proper.

Definition 6.3. For $\zeta \in \mathbb{C}$, the holomorphic symplectic manifold

$$
\mathcal{M}_{\zeta}^{\mathrm{irr}}\left(\mathcal{O}_{1}, \ldots, \mathcal{O}_{n}\right):=\left\{\begin{array}{l|l}
A(z) \in \prod_{i=1}^{n} \mathcal{O}_{i} & \begin{array}{l}
A(z) \text { is irreducible, } \\
\sum_{i} \operatorname{res}_{z=t_{i}} A(z)=-\zeta \operatorname{Id}_{V}
\end{array}
\end{array}\right\} / \operatorname{GL}(V)
$$

is called the $\zeta$-twisted naive moduli space of irreducible systems having a pole of truncated formal type $\mathcal{O}_{i}$ at each $t_{i}, i=1, \ldots, n$. In the 0 -twisted (untwisted) case, we simply write $\mathcal{M}_{0}^{\text {irr }}\left(\mathcal{O}_{1}, \ldots, \mathcal{O}_{n}\right) \equiv \mathcal{M}^{\text {irr }}\left(\mathcal{O}_{1}, \ldots, \mathcal{O}_{n}\right)$.

If we have a specific element $\Lambda_{i}(z) \in \mathcal{O}_{i}$ for each $i$, the following notation is also useful:

$$
\mathcal{M}_{\zeta}^{\mathrm{set}}\left(\Lambda_{1}, \ldots, \Lambda_{n}\right) \equiv \mathcal{M}_{\zeta}^{\mathrm{set}}\left(\mathcal{O}_{1}, \ldots, \mathcal{O}_{n}\right), \quad \mathcal{M}_{\zeta}^{\mathrm{irr}}\left(\Lambda_{1}, \ldots, \Lambda_{n}\right) \equiv \mathcal{M}_{\zeta}^{\mathrm{irr}}\left(\mathcal{O}_{1}, \ldots, \mathcal{O}_{n}\right)
$$

Remark 6.4. Recall that a holomorphic vector bundle with meromorphic connection $(E, \nabla)$ over a compact Riemann surface is stable if for any nonzero proper subbundle $F \subset E$ preserved by $\nabla$, the inequality $\operatorname{deg} F / \operatorname{rank} F<\operatorname{deg} E / \operatorname{rank} E$ holds. It is easy to see that if the base space is $\mathbb{P}^{1}$ and $E$ is trivial, then $(E, \nabla)$ is stable if and only if it has no nonzero proper trivial subbundle $F \subset E$ preserved by $\nabla$. This implies that a system $A(z) \in \bigoplus_{i} \mathfrak{g}_{k_{i}}^{*}(V)$ is irreducible if and only if the associated vector bundle with meromorphic connection $\left(\mathbb{P}^{1} \times V, \mathrm{~d}-A(z) \mathrm{d} z\right)$ is stable.

Remark 6.5. Let us recall a normal form $\Lambda(z)$ introduced in Remark 5.4. Assume that each $\Gamma_{\lambda}$ is non-resonant, i.e., no two distinct eigenvalues of $\Gamma_{\lambda}$ differ by an integer. Then one can show that an element $A(z) \in \mathfrak{g}_{k}^{*}(V)$ is equivalent to $\Lambda(z)$ under the coadjoint action if and only if there is a formal gauge transformation $g(z) \in \operatorname{Aut}_{\mathbb{C}[[z]]}(\mathbb{C}[[z]] \otimes V)$ which makes $\mathrm{d}-A(z) \mathrm{d} z$ into $\mathrm{d}-\Lambda(z) \mathrm{d} z$ (see [31, Remark 18]). In this sense the truncated formal type of $\Lambda(z)$ actually prescribe a formal type. Hence, if each $\mathcal{O}_{i} \subset \mathfrak{g}_{k_{i}}^{*}(V)$ contains some normal form with nonresonant residue parts, then the naive moduli space $\mathcal{M}^{\text {set }}\left(\mathcal{O}_{1}, \ldots, \mathcal{O}_{n}\right)$ gives the moduli space of meromorphic connections on the trivial bundle $\mathbb{P}^{1} \times V$ having a pole of prescribed formal type at each $t_{i}$.

\subsection{Star-shaped quivers of length one}

In some special case, the naive moduli space $\mathcal{M}^{\mathrm{irr}}\left(\mathcal{O}_{1}, \ldots, \mathcal{O}_{n}\right)$ can be described as a quiver variety. Suppose that for each $i=1, \ldots, n$, the coadjoint orbit $\mathcal{O}_{i}$ contains an element of the form

$$
\Xi_{i}(z)=\left(\begin{array}{cc}
\xi_{i}(z) \operatorname{Id}_{V_{i}} & 0 \\
0 & \eta_{i}(z) \operatorname{Id}_{V_{i}^{\prime}}
\end{array}\right)
$$


for some vector space decomposition $V=V_{i} \oplus V_{i}^{\prime}$ and distinct $\xi_{i}, \eta_{i} \in \mathfrak{g}_{k_{i}}^{*}(\mathbb{C})$. Let $d_{i}$ be the pole order of $\lambda_{i}:=\xi_{i}-\eta_{i}$. Note that $\Xi_{i}$ is a particular example of normal forms introduced in Remark 5.4, and it has non-resonant residue parts (see Remark 6.5) if and only if $d_{i}>1$ or $\underset{z=0}{\operatorname{res}}\left(\xi_{i}-\eta_{i}\right) \notin \mathbb{Z}$. Also, note that $\sum_{i=1}^{n} \operatorname{tr} \underset{z=0}{\operatorname{res}} \Xi_{i}(z)=0$ is a necessary condition for the nonemptiness of $\mathcal{M}^{\text {set }}\left(\Xi_{1}, \ldots, \Xi_{n}\right)=\mathcal{M}^{\text {set }}\left(\mathcal{O}_{1}, \ldots, \mathcal{O}_{n}\right)$. Indeed, if some $A(z)$ gives a point in $\mathcal{M}^{\text {set }}\left(\Xi_{1}, \ldots, \Xi_{n}\right)$, then

$$
0=\sum_{i=1}^{n} \operatorname{tr} \underset{z=t_{i}}{\operatorname{res}} A(z)=\sum_{i=1}^{n} \operatorname{tr} \underset{z=0}{\operatorname{res}} \Xi_{i}(z),
$$

since the function $\operatorname{tr} \circ \operatorname{res}_{z=0}: \mathfrak{g}_{k_{i}}^{*}(V) \rightarrow \mathbb{C}$ is invariant under the coadjoint action for each $i$.

Set $I:=\{0,1, \ldots, n\}$ and let $\mathrm{Q}=(I, \Omega, \mathrm{s}, \mathrm{t})$ be the 'star-shaped quiver with $n$ legs of length one' as drawn below

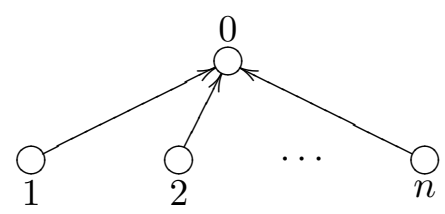

We set $V_{0}:=V, d_{0}:=1$, which together with the above $V_{i}, d_{i}$ give an $I$-graded $\mathbb{C}$-vector space $\mathbf{V}=\bigoplus_{i} V_{i}$ and multiplicities $\mathbf{d}=\left(d_{i}\right) \in \mathbb{Z}_{>0}^{I}$. Also we set

$$
\lambda_{0}(z):=z^{-1} \sum_{i=1}^{n} \operatorname{res}_{z=0} \eta_{i}(z) \in R^{1},
$$

which together with the above $\lambda_{i}$ gives an element $\lambda=\left(\lambda_{i}\right) \in R^{\mathbf{d}}$. Note that

$$
\begin{aligned}
\sum_{i=1}^{n} \operatorname{tr} \underset{z=0}{\operatorname{res}} \Xi_{i}(z) & =\sum_{i=1}^{n}\left[\left(\operatorname{dim} V_{i}\right) \underset{z=0}{\operatorname{res}} \lambda_{i}(z)+(\operatorname{dim} V) \underset{z=0}{\operatorname{res}} \eta_{i}(z)\right] \\
& =\sum_{i=1}^{n}\left(\operatorname{dim} V_{i}\right) \underset{z=0}{\operatorname{res}} \lambda_{i}(z)+(\operatorname{dim} V) \underset{z=0}{\operatorname{res}} \lambda_{0}(z)=\mathbf{v} \cdot \operatorname{res} \lambda
\end{aligned}
$$

where $\mathbf{v}:=\operatorname{dim} \mathbf{V}$. Hence $\sum_{i=1}^{n} \operatorname{tr} \underset{z=0}{\operatorname{res}} \Xi_{i}(z)=0$ if and only if $\mathbf{v} \cdot$ res $\lambda=0$, which is a necessary condition for the non-emptiness of $\mathcal{N}_{\mathbf{Q}, \mathbf{d}}^{\text {set }}(\lambda, \mathbf{v})$ (Proposition 3.4).

Proposition 6.6. There exists a bijection from $\mathcal{N}_{Q, \mathbf{d}}^{\text {set }}(\lambda, \mathbf{v})$ to $\mathcal{M}^{\mathrm{set}}\left(\Xi_{1}, \ldots, \Xi_{n}\right)$, which maps $\mathcal{N}_{\mathrm{Q}, \mathbf{d}}^{\mathrm{s}}(\lambda, \mathbf{v})$ symplectomorphically onto $\mathcal{M}^{\mathrm{irr}}\left(\Xi_{1}, \ldots, \Xi_{n}\right)$.

Proof. Set $\zeta:=\underset{z=0}{\operatorname{res}} \lambda_{0}(z)=\sum_{i=1}^{n} \underset{z=0}{\operatorname{res}} \eta_{i}(z)$. Then the scalar shift with $\eta_{i}$ induces a bijection $\mathcal{M}^{\text {set }}\left(\Xi_{1}, \ldots, \Xi_{n}\right) \rightarrow \mathcal{M}_{\zeta}^{\text {set }}\left(\Lambda_{1}, \ldots, \Lambda_{n}\right)$, where

$$
\Lambda_{i}(z):=\Xi_{i}(z)-\eta_{i}(z) \operatorname{Id}_{V}=\left(\begin{array}{cc}
\lambda_{i}(z) \operatorname{Id}_{V_{i}} & 0 \\
0 & 0 \operatorname{Id}_{V_{i}^{\prime}}
\end{array}\right) \in \mathfrak{g}_{d_{i}}^{*}(V) \subset \mathfrak{g}_{k_{i}}^{*}(V)
$$

and it preserves the irreducibility. As $\Lambda_{i}$ has the pole order $d_{i}$, the $G_{k_{i}}(V)$-action on $\Lambda_{i}$ reduces to the $G_{d_{i}}(V)$-action via the natural projection $G_{k_{i}}(V) \rightarrow G_{d_{i}}(V)$, so that the orbit $G_{k_{i}}(V) \cdot \Lambda_{i}=$ $G_{d_{i}}(V) \cdot \Lambda_{i}$ is a $G_{d_{i}}(V)$-coadjoint orbit. This replacement of order has no effect on the naive moduli space. 
By the definition of $\mathrm{Q}$, we have $\widehat{V}_{i}=V_{0} \otimes_{\mathbb{C}} R_{1}=V$ for each $i>0$ and

$$
\mathbf{M}_{\mathbf{Q}, \mathbf{d}}(\mathbf{V})=\bigoplus_{i=1}^{n} M_{i}, \quad M_{i}:=\operatorname{Hom}\left(V, V_{i} \otimes_{\mathbb{C}} R_{d_{i}}\right) \oplus \operatorname{Hom}\left(V_{i} \otimes_{\mathbb{C}} R_{d_{i}}, V\right) .
$$

Now consider the sets $Z_{i} \subset M_{i}, i>0$ given in Lemma 3.6. Since the top coefficients of $\lambda_{i} \in$ $\mathfrak{g}_{d_{i}}^{*}(\mathbb{C}), i>0$ are nonzero, Lemma 3.5 implies that for each $i>0$, any point in $\mu_{\mathbf{d}, i}^{-1}\left(\lambda_{i}(z) \operatorname{Id}_{V_{i}}\right)$ satisfies condition (3.4). Hence

$$
\bigcap_{i=1}^{n} \mu_{\mathbf{d}, i}^{-1}\left(\lambda_{i}(z) \operatorname{Id}_{V_{i}}\right)=\prod_{i=1}^{n} Z_{i}
$$

Since $\operatorname{dim} V_{i} \leq \operatorname{dim} V$ for all $i>0$, Lemmas 3.6 and 3.7 imply that the map

$$
\Phi=\left(\Phi_{i}\right): \quad \mathbf{M}_{\mathbf{Q}, \mathbf{d}}(\mathbf{V}) \rightarrow \bigoplus_{i=1}^{n} \mathfrak{g}_{d_{i}}^{*}(V), \quad B \mapsto\left(-B_{\leftarrow i}\left(z \operatorname{Id}_{V_{i} \otimes R_{d_{i}}}-N_{i}\right)^{-1} B_{i \leftarrow}\right)
$$

induces a symplectomorphism

$$
\bigcap_{i=1}^{n} \mu_{\mathbf{d}, i}^{-1}\left(\lambda_{i}(z) \operatorname{Id}_{V_{i}}\right) / \prod_{i=1}^{n} G_{d_{i}}\left(V_{i}\right)=\prod_{i=1}^{n}\left(Z_{i} / G_{d_{i}}\left(V_{i}\right)\right) \stackrel{\simeq}{\longrightarrow} \prod_{i=1}^{n} G_{d_{i}}(V) \cdot \Lambda_{i},
$$

which is clearly $\mathrm{GL}(V)$-equivariant. Note that

$$
\sum_{i=1}^{n} \operatorname{res}_{z=0}\left(-B_{\leftarrow i}\left(z \operatorname{Id}_{V_{i} \otimes R_{d_{i}}}-N_{i}\right)^{-1} B_{i \leftarrow}\right)=-\sum_{i=1}^{n} B_{\leftarrow i} B_{i \leftarrow}=\operatorname{res}_{z=0} \mu_{\mathbf{d}, 0}(B) .
$$

Taking the (set-theoretical) symplectic quotient by the $\mathrm{GL}(V)$-action at $-\zeta \mathrm{Id}_{V}$, we thus obtain a bijection from $\mathcal{N}_{\mathbf{Q}, \mathbf{d}}^{\text {set }}(\lambda, \mathbf{v})$ to $\mathcal{M}_{\zeta}^{\text {set }}\left(\Lambda_{1}, \ldots, \Lambda_{n}\right)$.

The proof of what it maps $\mathcal{N}_{Q, \mathbf{d}}^{\mathrm{s}}(\lambda, \mathbf{v})$ onto $\mathcal{M}_{\zeta}^{\mathrm{irr}}\left(\Lambda_{1}, \ldots, \Lambda_{n}\right)$ is quite similar to Lemma 5.9. First, assume that a point $B \in \mu_{\mathbf{d}}^{-1}\left(-\lambda \operatorname{Id}_{\mathbf{V}}\right)$ is stable. Let $\Phi(B)=\left(\sum_{l=1}^{d_{i}} A_{i, l} z^{-l}\right)$, and assume further that a subspace $S_{0} \subset V$ is invariant under all $A_{i, l}$. We define

$$
S_{i}:=\sum_{l=1}^{d_{i}} N_{i}^{l-1} B_{i \leftarrow}\left(S_{0}\right), \quad i>0,
$$

and set $\mathbf{S}:=\bigoplus_{i \in I} S_{i} \subset \mathbf{V}_{\mathbf{d}}$. Then $N_{i}\left(S_{i}\right) \subset S_{i}, B_{i \leftarrow}\left(S_{0}\right) \subset S_{i}$ and

$$
B_{\leftarrow i}\left(S_{i}\right)=\sum_{l} B_{\leftarrow i} N_{i}^{l-1} B_{i \leftarrow}\left(S_{0}\right)=\sum_{l} A_{i, l}\left(S_{0}\right) \subset S_{0}
$$

imply that $\mathbf{S}$ is $B$-invariant. Since $B$ is stable, we thus have $\mathbf{S}=0$ or $\mathbf{S}=\mathbf{V}_{\mathbf{d}}$, and in particular, $S_{0}=0$ or $S_{0}=V$, which shows that the system $\Phi(B)$ is irreducible.

Conversely, assume that the system $\Phi(B)=\left(\sum_{l} A_{i, l} z^{-l}\right)$ is irreducible. Let $\mathbf{S}=\bigoplus_{i} S_{i}$ be a $B$-invariant subspace of $\mathbf{V}_{\mathbf{d}}$ satisfying $N_{i}\left(S_{i}\right) \subset S_{i}$ for all $i \in I$. Then $S_{0}$ is invariant under all $A_{i, l}$, and hence $S_{0}=0$ or $S_{0}=V$. If $S_{0}=0$, then for each $i>0$, we have $B_{\leftarrow i}\left(S_{i}\right)=0$, which implies $S_{i}=0$ since $\operatorname{Ker} B_{\leftarrow i} \cap \operatorname{Ker} N_{i}=0$ by Lemma 3.5 and $\left.N_{i}\right|_{S_{i}}$ is nilpotent. Dualizing the argument, we easily see that $S_{i}=V_{i} \otimes_{\mathbb{C}} R_{d_{i}}, i>0$ if $S_{0}=V$. Hence $\mathbf{S}=0$ or $\mathbf{S}=\mathbf{V}_{\mathbf{d}}$, which shows that $B$ is stable. 
Conversely, let $\mathrm{Q}=(I, \Omega, \mathrm{s}, \mathrm{t})$ be as above and suppose that an $I$-graded $\mathbb{C}$-vector space $\mathbf{V}=$ $\bigoplus_{i} V_{i}$ and multiplicities $\mathbf{d}=\left(d_{i}\right)$ are given. Suppose further that they satisfy $\operatorname{dim} V_{i} \leq \operatorname{dim} V_{0}$ and $d_{0}=1$. Set $V:=V_{0}$, and fix a $\mathbb{C}$-vector space $V_{i}^{\prime}$ of $\operatorname{dimension} \operatorname{dim} V-\operatorname{dim} V_{i}$ together with an identification $V \simeq V_{i} \oplus V_{i}^{\prime}$ for each $i>0$. Also, for each $\lambda \in R^{\mathrm{d}}$, set $\zeta:=\operatorname{res}_{z=0} \lambda_{0}$ and let $\Lambda_{i}$ be as in (6.2). Then the above proof also shows that the map $\Phi$ given in (6.3) induces a bijection $\mathcal{N}_{\mathbf{Q}, \mathbf{d}}^{\text {set }}(\lambda, \mathbf{v}) \rightarrow \mathcal{M}_{\zeta}^{\text {set }}\left(\Lambda_{1}, \ldots, \Lambda_{n}\right)$ mapping $\mathcal{N}_{\mathbf{Q}, \mathbf{d}}^{\text {s }}(\lambda, \mathbf{v})$ symplectomorphically onto $\mathcal{M}_{\zeta}^{\text {irr }}\left(\Lambda_{1}, \ldots, \Lambda_{n}\right)$.

\subsection{Middle convolution}

Recall the map given in (6.3);

$$
\Phi=\left(\Phi_{i}\right): \quad \mathbf{M}_{\mathbf{Q}, \mathbf{d}}(\mathbf{V}) \rightarrow \bigoplus_{i=1}^{n} \mathfrak{g}_{d_{i}}^{*}(V), \quad B \mapsto\left(-B_{\leftarrow i}\left(z \operatorname{Id}_{V_{i} \otimes R_{d_{i}}}-N_{i}\right)^{-1} B_{i \leftarrow}\right) .
$$

Noting $\widehat{V}_{0}=\bigoplus_{i=1}^{n} V_{i} \otimes R_{d_{i}}$, we set

$$
T:=\bigoplus_{i=1}^{n}\left(t_{i} \operatorname{Id}_{V_{i} \otimes R_{d_{i}}}+N_{i}\right) \in \operatorname{End}\left(\widehat{V}_{0}\right)
$$

Using the natural inclusion $\iota_{i}: V_{i} \otimes R_{d_{i}} \rightarrow \widehat{V}_{0}$ and projection $\pi_{i}: \widehat{V}_{0} \rightarrow V_{i} \otimes R_{d_{i}}$, we then have

$$
\left(z \operatorname{Id}_{\widehat{V}_{0}}-T\right)^{-1}=\sum_{i=1}^{n} \iota_{i}\left(z-t_{i}-N_{i}\right)^{-1} \pi_{i}=\sum_{i=1}^{n} \sum_{j=1}^{k_{i}}\left(z-t_{i}\right)^{-j} \iota_{i} N_{i}^{j-1} \pi_{i} .
$$

Thus we can write the systems $\Phi(B)$ as

$$
\Phi(B)=-\sum_{i=1}^{n} B_{\leftarrow i}\left(\left(z-t_{i}\right) \operatorname{Id}_{V_{i} \otimes R_{d_{i}}}-N_{i}\right)^{-1} B_{i \leftarrow}=B_{0 \leftarrow}\left(z \operatorname{Id}_{\widehat{V}_{0}}-T\right)^{-1} B_{\leftarrow 0} .
$$

Such an expression of systems has been familiar since Harnad's work [10], and is in fact quite useful to formulate the so-called middle convolution [31], which was originally introduced by Katz [14] for local systems on a punctured $\mathbb{P}^{1}$ and generalized by Arinkin [1] for irregular $\mathcal{D}$ modules.

Let us define the generalized middle convolution according to [31]. First, we introduce the following fact, which is a refinement of Woodhouse and Kawakami's observation [30, 15]:

Proposition 6.7 ([31, Propositions 1 and 2]). Under the assumption $V \neq 0$, for any system $A(z)$ with poles at $t_{i}, i=1,2, \ldots, n$ and possibly a simple pole at $\infty$, there exists a quadruple $(W, T, X, Y)$ consisting of

- a finite-dimensional $\mathbb{C}$-vector space $W$;

- an endomorphism $T \in \operatorname{End}(W)$ with eigenvalues $t_{i}, i=1,2, \ldots, n$;

- a pair of homomorphisms $(X, Y) \in \operatorname{Hom}(W, V) \oplus \operatorname{Hom}(V, W)$,

such that

$$
\begin{aligned}
& X\left(z \operatorname{Id}_{W}-T\right)^{-1} Y=A(z), \\
& \operatorname{Ker} X_{i} \cap \operatorname{Ker} N_{i}=0, \quad \operatorname{Im} Y_{i}+\operatorname{Im} N_{i}=V,
\end{aligned}
$$


where $N_{i}$ is the nilpotent part of $T$ restricted on its generalized $t_{i}$-eigenspace $W_{i}:=\operatorname{Ker}(T-$ $\left.t_{i} \operatorname{Id}_{W}\right)^{\operatorname{dim} W}$, and $\left(X_{i}, Y_{i}\right) \in \operatorname{Hom}\left(W_{i}, V\right) \oplus \operatorname{Hom}\left(V, W_{i}\right)$ is the block component of $(X, Y)$ with respect to the decomposition $W=\bigoplus_{i} W_{i}$. Moreover the choice of $(W, T, X, Y)$ is unique in the following sense: if two quadruples $(W, T, X, Y)$ and $\left(W^{\prime}, T^{\prime}, X^{\prime}, Y^{\prime}\right)$ satisfy (6.5) and (6.6), then there exists an isomorphism $f: W \rightarrow W^{\prime}$ such that

$$
f T f^{-1}=T^{\prime}, \quad X=X^{\prime} f, \quad f Y=Y^{\prime} .
$$

The above enables us to define the middle convolution. For a system $A(z)=\left(A_{i}\right) \in$ $\bigoplus_{i=1}^{n} \mathfrak{g}_{k_{i}}^{*}(V)$, take a quadruple $(W, T, X, Y)$ satisfying (6.5) and (6.6). Then for given $\zeta \in \mathbb{C}$, set $V^{\zeta}:=W / \operatorname{Ker}\left(Y X+\zeta \operatorname{Id}_{W}\right)$ and let

- $X^{\zeta}: W \rightarrow V^{\zeta}$ be the projection;

- $Y^{\zeta}: V^{\zeta} \rightarrow W$ be the injection induced from $Y X+\zeta \operatorname{Id}_{W}$.

Now we define

$$
m c_{\zeta}(A):=X^{\zeta}\left(z \operatorname{Id}_{W}-T\right)^{-1} Y^{\zeta} \in \bigoplus_{i=1}^{n} \mathfrak{g}_{k_{i}}^{*}\left(V^{\zeta}\right) .
$$

By virtue of Proposition 6.7, the equivalence class of $m c_{\zeta}(A)$ under constant gauge transformations depends only on that of $A(z)$. We call it the middle convolution of $A(z)$ with $\zeta .^{5}$

Let us come back to our situation. The expression (6.4) and Lemma 3.5 (which we apply for all $i>0)$ imply that the quadruple $\left(\widehat{V}_{0}, T, B_{0 \leftarrow}, B_{\leftarrow 0}\right)$ satisfies $(6.5)$ and $(6.6)$ for $A(z)=\Phi(B)$. Now assume $\lambda_{0}(z) \neq 0$ and consider the middle convolution $m c_{\zeta}(A)$ with $\zeta:=\underset{z=0}{\operatorname{res}} \lambda_{0}$. By the definition, the triple $\left(V^{\zeta}, B_{0 \leftarrow}^{\zeta}, B_{\leftarrow 0}^{\zeta}\right)$ satisfies

$$
\begin{aligned}
& B_{\leftarrow 0}^{\zeta} B_{0 \leftarrow}^{\zeta}=B_{\leftarrow 0} B_{0 \leftarrow}+\zeta \operatorname{Id}_{\widehat{V}_{0}}, \\
& \operatorname{Ker} B_{\leftarrow 0}^{\zeta}=0, \quad \operatorname{Im} B_{0 \leftarrow}^{\zeta}=V^{\zeta},
\end{aligned}
$$

i.e., it provides a full-rank decomposition of the matrix $B_{\leftarrow 0} B_{0 \leftarrow}+\zeta \operatorname{Id}_{\widehat{V}_{0}}$. Recall that such a triple already appeared in Section 4; conditions (4.3) and (4.4) for the 0-th reflection functor $\mathcal{F}_{0}$ imply that if we take an $I$-graded $\mathbb{C}$-vector space $\mathbf{V}^{\prime}=\bigoplus_{i} V_{i}^{\prime}$ with $\operatorname{dim} \mathbf{V}^{\prime}=s_{0}(\mathbf{v})$ as in Section 4.2 and a representative $B^{\prime} \in \mathbf{M}_{\mathbf{Q}, \mathbf{d}}\left(\mathbf{V}^{\prime}\right)$ of $\mathcal{F}_{0}[B] \in \mathcal{N}_{\mathbf{Q}, \mathbf{d}}^{\mathbf{s}}\left(r_{0}(\lambda), s_{0}(\mathbf{v})\right)$, then the triple $\left(V_{0}^{\prime}, B_{0 \leftarrow}^{\prime}, B_{\leftarrow 0}^{\prime}\right)$ also satisfies (6.7) and (6.8) (note that $d_{0}=1$ and $N_{0}=0$ ). By the uniqueness of the full-rank decomposition, we then see that there exists an isomorphism $f: V^{\zeta} \rightarrow V_{0}^{\prime}$ such that $B_{0 \leftarrow}^{\prime}=f B_{0 \leftarrow}^{\zeta}$, $B_{\leftarrow 0}^{\prime}=B_{\leftarrow 0}^{\zeta} f^{-1}$, and hence

$$
\Phi\left(B^{\prime}\right)=B_{0 \leftarrow}^{\prime}\left(z \operatorname{Id}_{\widehat{V}_{0}}-T\right)^{-1} B_{\leftarrow 0}^{\prime}=f B_{0 \leftarrow}^{\zeta}\left(z \operatorname{Id}_{\widehat{V}_{0}}-T\right)^{-1} B_{\leftarrow 0}^{\zeta} f^{-1}=f m c_{\zeta}(A) f^{-1} .
$$

The arguments in the previous subsection for $\mathbf{V}^{\prime}, \lambda^{\prime}:=r_{0}(\lambda)$ show that $\Phi: \mathbf{M}_{\mathbf{Q}, \mathbf{d}}\left(\mathbf{V}^{\prime}\right) \rightarrow$ $\bigoplus_{i=1}^{n} \mathfrak{g}_{d_{i}}^{*}\left(V_{0}^{\prime}\right)$ induces a bijection between $\mathcal{N}_{\mathbf{Q}, \mathbf{d}}^{\text {set }}\left(r_{0}(\lambda), s_{0}(\mathbf{v})\right)$ and $\mathcal{M}_{-\zeta}^{\text {set }}\left(\Lambda_{1}^{\prime}, \ldots, \Lambda_{n}^{\prime}\right)$, where

$$
\Lambda_{i}^{\prime}(z)=\left(\begin{array}{cc}
\lambda_{i}^{\prime}(z) \operatorname{Id}_{V_{i}} & 0 \\
0 & 0 \operatorname{Id}_{V_{i}^{\prime \prime}}
\end{array}\right) \in \mathfrak{g}_{d_{i}}^{*}\left(V_{0}^{\prime}\right), \quad V_{0}^{\prime} \simeq V_{i} \oplus V_{i}^{\prime \prime}
$$

We have now proved the following:

\footnotetext{
${ }^{5}$ In [31], an explicit construction of the quadruple $(W, T, X, Y)$ is given so that the middle convolution $m c_{\zeta}(A)$ is well-defined as a system, not as a gauge equivalence class.
} 
Proposition 6.8. Let $(\mathrm{Q}, \mathbf{d}), \lambda, \mathbf{v}$ be as in Proposition 6.6, and assume $\zeta:=\underset{z=0}{\operatorname{res}} \lambda_{0}$ is nonzero. Under the above notation, one then has the following commutative diagram:

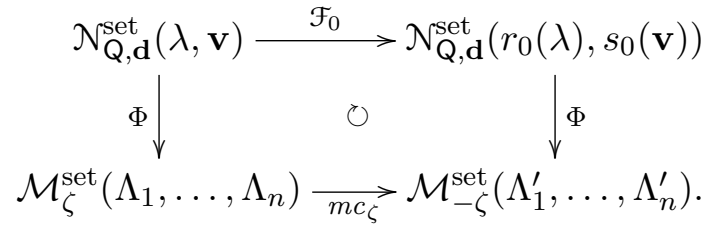

Next, consider the reflection functors $\mathcal{F}_{i}$ for $i>0$. Let $\left[B^{\prime}\right]=\mathcal{F}_{i}[B]$. Then condition $(4.2)$ implies

$$
B_{\leftarrow j}^{\prime}\left(z \operatorname{Id}_{V_{j} \otimes R_{d_{j}}}-N_{j}\right)^{-1} B_{j \leftarrow}^{\prime}=B_{\leftarrow j}\left(z \operatorname{Id}_{V_{j} \otimes R_{d_{j}}}-N_{j}\right)^{-1} B_{j \leftarrow}, \quad j \neq 0, i,
$$

which together with (4.3) shows that the two systems $\Phi(B)$ and $\Phi^{\prime}(B)$ are related via

$$
\Phi\left(B^{\prime}\right)=\Phi(B)-\lambda_{i}\left(z-t_{i}\right) \operatorname{Id}_{V} .
$$

Proposition 6.9. Let $(\mathrm{Q}, \mathbf{d}), \lambda, \mathbf{v}$ be as in Proposition 6.6, and set $\zeta:=\operatorname{res}_{z=0} \lambda_{0}$. For $i=$ $1,2, \ldots, n$, one then has the following commutative diagram:

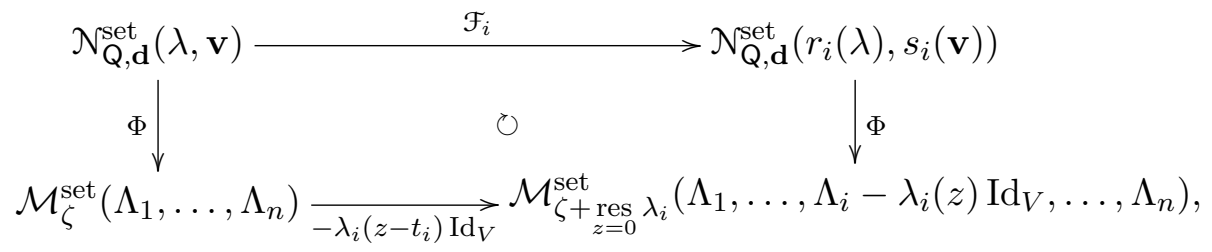

where the bottom horizontal arrow is given by the shift $A(z) \mapsto A(z)-\lambda_{i}\left(z-t_{i}\right) \operatorname{Id}_{V}$.

Remark 6.10. In [10], Harnad considered two meromorphic connections having the following symmetric description:

$$
\nabla=\mathrm{d}-\left(S+X\left(z \operatorname{Id}_{W}-T\right)^{-1} Y\right) \mathrm{d} z, \quad \nabla^{\prime}=\mathrm{d}+\left(T+Y\left(z \operatorname{Id}_{V}-S\right)^{-1} X\right) \mathrm{d} z,
$$

where $V, W$ are finite-dimensional $\mathbb{C}$-vector spaces, $S, T$ are regular semisimple endomorphisms of $V, W$ respectively, and $(X, Y) \in \operatorname{Hom}(W, V) \oplus \operatorname{Hom}(V, W)$ such that both $(W, T, X, Y)$ and $(V, S, Y, X)$ satisfy (6.6). These have an order 2 pole at $z=\infty$ and simple poles at the eigenvalues of $T, S$ respectively. He then proved that the isomonodromic deformations of the two systems are equivalent. After his work, such a duality, called the Harnad duality, was established in more general cases by Woodhouse [30].

Note that if $S=0$, we have $\nabla^{\prime}=\mathrm{d}+z^{-1} P Q \mathrm{~d} z$. Hence on the 'dual side', the operation $m c_{\zeta}$ corresponds to just the scalar shift by $z^{-1} \zeta \mathrm{d} z$. This interpretation enables us to generalize the middle convolution further; see [31].

\subsection{Examples: rank two cases}

The case $\operatorname{dim} V=2$ is most important because in this case a generic element in $\mathfrak{g}_{k_{i}}^{*}(V)$ can be transformed into an element of the form $\Xi_{i}(z)=\xi_{i}(z) \oplus \eta_{i}(z)$ for some distinct $\xi_{i}, \eta_{i} \in \mathfrak{g}_{k_{i}}^{*}(\mathbb{C})$. The dimension of $\mathcal{M}^{\text {irr }}\left(\Xi_{1}, \ldots, \Xi_{n}\right)$ can be computed as

$$
\operatorname{dim} \mathcal{M}^{\mathrm{irr}}\left(\Xi_{1}, \ldots, \Xi_{n}\right)=\operatorname{dim} \mathcal{N}_{\mathrm{Q}, \mathbf{d}}^{\mathrm{s}}(\lambda, \mathbf{v})=2-(\mathbf{v}, \mathbf{v})=2 \sum_{i=1}^{n} d_{i}-6
$$

if it is nonempty. 
First, consider the case $\operatorname{dim} \mathcal{M}^{\mathrm{irr}}\left(\Xi_{1}, \ldots, \Xi_{n}\right)=0$. The above formula implies that the tuple $\left(d_{1}, \ldots, d_{n}\right)$ must be one of the following (up to permutation on indices):

$$
(1,1,1), \quad(2,1), \quad(3) .
$$

The corresponding $(\mathbf{Q}, \mathbf{d})$ have the underlying graphs with multiplicities given by the picture below

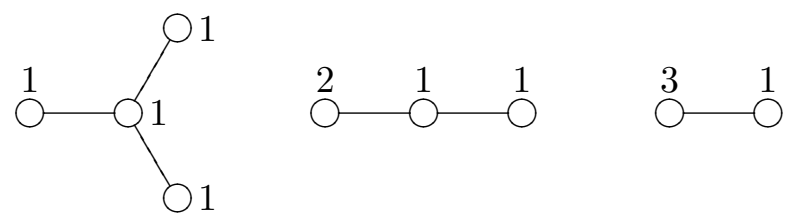

The associated Kac-Moody algebras are respectively given by

$$
D_{4}, \quad C_{3}, \quad G_{2}
$$

From Example 5.6, we see that the effect of normalization on these quivers with multiplicities is given as follows:

$$
D_{4} \leftarrow C_{4}, \quad C_{3} \rightarrow A_{3}, \quad G_{2} \rightarrow A_{2},
$$

where the arrows represent the process of normalization.

Next consider the case $\operatorname{dim} \mathcal{M}^{\operatorname{irr}}\left(\Xi_{1}, \ldots, \Xi_{n}\right)=2$. Then the tuple $\left(d_{1}, \ldots, d_{n}\right)$ must be one of the following (up to permutation on indices):

$$
(1,1,1,1), \quad(2,1,1), \quad(3,1), \quad(2,2), \quad(4) .
$$

The corresponding $(\mathbf{Q}, \mathbf{d})$ have the underlying graphs with multiplicities given by the picture below
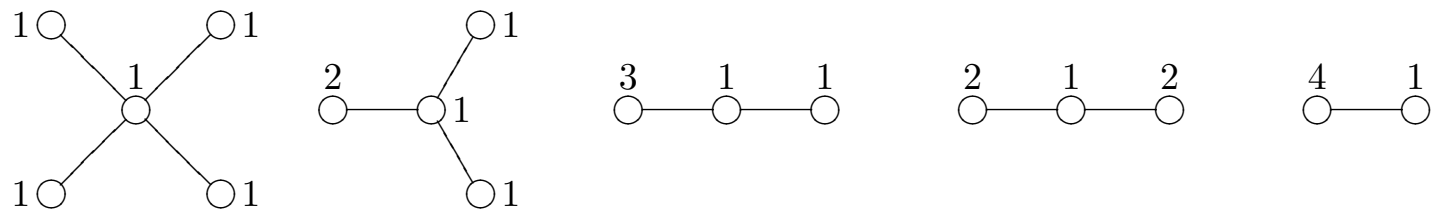

The associated Kac-Moody algebras are respectively given by

$$
D_{4}^{(1)}, \quad A_{5}^{(2)}, \quad D_{4}^{(3)}, \quad C_{2}^{(1)}, \quad A_{2}^{(2)} .
$$

From Example 5.6, we see that the effect of normalization on these quivers with multiplicities is given as follows:

$$
D_{4}^{(1)} \leftarrow A_{7}^{(2)} \leftarrow C_{4}^{(1)}, \quad A_{3}^{(1)} \leftarrow A_{5}^{(2)} \leftarrow C_{3}^{(1)}, \quad D_{4}^{(3)} \rightarrow A_{2}^{(1)}, \quad C_{2}^{(1)} \rightarrow D_{3}^{(2)}, \quad A_{2}^{(2)} \rightarrow A_{1}^{(1)},
$$

where the arrows represent the process of normalization. Hence by performing the normalization if necessary, we obtain the following list of (untwisted) affine Lie algebras:

$$
D_{4}^{(1)}, \quad A_{3}^{(1)}, \quad A_{2}^{(1)}, \quad C_{2}^{(1)}, \quad A_{1}^{(1)},
$$

which is well-known as the list of Okamoto's affine Weyl symmetry groups of the Painlevé equations of type VI, V, ..., II, as mentioned in Introduction. 
Remark 6.11. In all the cases appearing in (6.9), we can check that $\mathcal{M}^{\mathrm{irr}}\left(\Xi_{1}, \ldots, \Xi_{n}\right)$ is nonempty if and only if $\sum_{i=1}^{n} \operatorname{tr} \underset{z=0}{\operatorname{res}} \Xi_{i}=0$ (recall that the 'only if' part is always true). We sketch the proof of the 'if' part below.

If $\left(d_{1}, \ldots, d_{n}\right) \neq(2,2)$, the naive moduli space $\mathcal{M}^{\mathrm{irr}}\left(\Xi_{1}, \ldots, \Xi_{n}\right)$ is isomorphic to an ordinal quiver variety $\mathcal{N}_{Q}^{\mathrm{s}}(\zeta, \mathbf{v})$ for some extended Dynkin quiver $\mathrm{Q}$ and $\zeta, \mathbf{v}$ as discussed above. The formulas (5.4) and (6.1) imply $\zeta \cdot \mathbf{v}=\sum_{i=1}^{n} \operatorname{tr} \underset{z=0}{\operatorname{ras}} \Xi_{i}$. Furthermore, since the expected dimension of $\mathcal{N}_{Q}^{\mathrm{s}}(\zeta, \mathbf{v})$ is two, we have $(\mathbf{v}, \mathbf{v})=0$, which implies that $\mathbf{v}$ is a (positive) imaginary root (see [13, Proposition 5.10]). In fact, $\mathbf{v}$ is the minimal positive imaginary root $\delta$ because at least one of its components is equal to one. It is known [17] that if $\zeta \cdot \delta=0$, then $\mathcal{N}_{\mathrm{Q}}^{\mathrm{s}}(\zeta, \delta)$ is a deformation of a Kleinian singularity, which is indeed nonempty ${ }^{6}$.

Now assume $\left(d_{1}, \ldots, d_{n}\right)=(2,2)$ and $\sum_{i=1}^{2} \operatorname{tr} \underset{z=0}{\operatorname{res}} \Xi_{i}=0$. Let $\lambda_{i}(z)=\lambda_{i, 2} z^{-2}+\lambda_{i, 1} z^{-1}, \Lambda_{i}(z)$, $\zeta$ be as in the proof of Proposition 6.6, and for instance, set

$$
\begin{aligned}
A_{1}(z) & :=\left(\begin{array}{cc}
2 \lambda_{1,2} & -2 \lambda_{1,2} \\
\lambda_{1,2} & -\lambda_{1,2}
\end{array}\right) z^{-2}+\left(\begin{array}{cc}
\lambda_{1,1}+\zeta & -\lambda_{1,1}-\zeta \\
\zeta & -\zeta
\end{array}\right) z^{-1} \\
& =\left(\begin{array}{ll}
2 & 1 \\
1 & 1
\end{array}\right)\left\{\Lambda_{1}(z)+\left(\begin{array}{cc}
0 & 0 \\
\zeta-\lambda_{1,1} & 0
\end{array}\right) z^{-1}\right\}\left(\begin{array}{ll}
2 & 1 \\
1 & 1
\end{array}\right) \\
A_{2}(z) & :=\Lambda_{2}(z)+\left(\begin{array}{cc}
0 & \lambda_{1,1}+\zeta \\
-\zeta & 0
\end{array}\right) z^{-1}
\end{aligned}
$$

For each $i$, using the assumption $\lambda_{i, 2} \neq 0$ and the formula

$$
\left(\begin{array}{cc}
1 & a z \\
b z & 1
\end{array}\right) \cdot \Lambda_{i}=\Lambda_{i}(z)+\left(\begin{array}{cc}
0 & b \lambda_{i, 2} \\
-a \lambda_{i, 2} & 0
\end{array}\right) z^{-1}, \quad a, b \in \mathbb{C},
$$

we easily see that $A_{i}(z)$ is contained in the $G_{2}\left(\mathbb{C}^{2}\right)$-coadjoint orbit through $\Lambda_{i}(z)$. Furthermore, the assumption $\sum_{i}$ tr res $\Xi_{z=0}=0$ implies $\lambda_{1,1}+\lambda_{2,1}=-2 \zeta$, and hence

$$
\underset{z=0}{\operatorname{res}} A_{1}(z)+\underset{z=0}{\operatorname{res}} A_{2}(z)=\left(\begin{array}{cc}
\lambda_{1,1}+\lambda_{2,1}+\zeta & 0 \\
0 & -\zeta
\end{array}\right)=-\zeta \operatorname{Id}_{\mathbb{C}^{2}} .
$$

The assumption $\lambda_{i, 2} \neq 0$ also implies that the top coefficients of $A_{1}(z), A_{2}(z)$ have no common eigenvector, which shows that the system $\left(A_{1}, A_{2}\right)$ is irreducible. Therefore the system $\left(A_{1}+\right.$ $\left.\eta_{1}(z) \operatorname{Id}_{\mathbb{C}^{2}}, A_{2}+\eta_{2}(z) \operatorname{Id}_{\mathbb{C}^{2}}\right)$ gives a point in $\mathcal{M}^{\text {irr }}\left(\Xi_{1}, \Xi_{2}\right)$.

Remark 6.12. Our list (6.10) of Dynkin diagrams is obtained from Sasano's on [29, p. 352] by taking the transpose of the generalized Cartan matrices. It is an interesting problem to ask the relation between our symmetries and Sasano's.

\section{A Appendix on normalization}

In this appendix, we prove Lemmas 5.2 and 5.3. Recall the situation discussed in Section 5.1; $i \in I$ is a fixed pole vertex with base $j$, and $\mathcal{O}$ is the $G_{d_{i}}\left(V_{j}\right)$-coadjoint orbit through

$$
\Lambda(z)=\left(\begin{array}{cc}
\lambda_{i}(z) \operatorname{Id}_{V_{i}} & 0 \\
0 & 0 \operatorname{Id}_{V_{j} / V_{i}}
\end{array}\right), \quad V_{j} \simeq V_{i} \oplus V_{j} / V_{i}
$$

where the top coefficient $\lambda_{i, d_{i}}$ of $\lambda_{i}(z)$ is assumed to be nonzero. Its 'normalized orbit' $\check{\mathcal{O}}$ is the $B_{d_{i}}\left(V_{j}\right)$-coadjoint orbit through the residue-free part $\Lambda^{0}$ of $\Lambda$.

\footnotetext{
${ }^{6}$ As a more direct proof, one can check that if $\zeta \cdot \delta=0$, then $(\zeta, \delta)$ satisfies the necessary and sufficient condition for the non-emptiness of $\mathcal{N}_{\mathrm{Q}}^{\mathrm{s}}(\zeta, \mathbf{v})$ given in [6, Theorem 1.2].
} 


\section{A.1 Proof of Lemma 5.2}

We check that the $B_{d_{i}}\left(V_{j}\right)$-coadjoint orbit $\check{\mathcal{O}}$ is invariant under the conjugation action by $K$, and is $K$-equivariantly symplectomorphic to the symplectic vector space

$$
\operatorname{Hom}\left(V_{j} / V_{i}, V_{i}\right)^{\oplus\left(d_{i}-2\right)} \oplus \operatorname{Hom}\left(V_{i}, V_{j} / V_{i}\right)^{\oplus\left(d_{i}-2\right)} .
$$

Note that all the coefficients of $\Lambda^{0}$ are fixed by $K$, and that the subset $B_{d_{i}}\left(V_{j}\right) \subset G_{d_{i}}\left(V_{j}\right)$ is invariant under the conjugation by constant matrices. Hence for any $k \in K$ and $g(z) \in B_{d_{i}}\left(V_{j}\right)$,

$$
k\left(g \cdot \Lambda^{0}\right) k^{-1}=\left(k g k^{-1}\right) \cdot\left(k \Lambda^{0} k^{-1}\right)=\left(k g k^{-1}\right) \cdot \Lambda^{0} \in \check{\mathcal{O}},
$$

i.e., $\check{\mathcal{O}}$ is invariant under the conjugation by $K$. Let us calculate the stabilizer of $\Lambda^{0}(z)$ with respect to the coadjoint $B_{d_{i}}\left(V_{j}\right)$-action. Suppose that $g(z) \in B_{d_{i}}\left(V_{j}\right)$ stabilizes $\Lambda^{0}(z)$. By the definition, we then have

$$
g(z) \Lambda^{0}(z)=\Lambda^{0}(z) g(z) \bmod z^{-1} \mathfrak{g l}\left(V_{j}\right)[[z]] .
$$

Write

$$
g(z)=\left(\begin{array}{ll}
G_{11}(z) & G_{12}(z) \\
G_{21}(z) & G_{22}(z)
\end{array}\right)
$$

according to the decomposition $V_{j}=V_{i} \oplus V_{j} / V_{i}$, and let $\lambda_{i}^{0}(z)$ be the residue-free part of $\lambda_{i}(z)$. Then

$$
\left[\Lambda^{0}(z), g(z)\right]=\left[\left(\begin{array}{cc}
\lambda_{i}^{0} \operatorname{Id}_{V_{i}} & 0 \\
0 & 0 \operatorname{Id}_{V_{j} / V_{i}}
\end{array}\right),\left(\begin{array}{ll}
G_{11} & G_{12} \\
G_{21} & G_{22}
\end{array}\right)\right]=\left(\begin{array}{cc}
0 & \lambda_{i}^{0} G_{12} \\
-\lambda_{i}^{0} G_{21} & 0
\end{array}\right) .
$$

Therefore (A.1) is equivalent to

$$
\lambda_{i}^{0}(z) f(z) \in z^{-1} \mathbb{C}[[z]]
$$

for all the matrix entries $f(z)=\sum_{k=1}^{d_{i}-1} f_{k} z^{k}$ of $G_{12}(z)$ and $G_{21}(z)$. We can write the above condition as

$$
\left(\begin{array}{cccc}
\lambda_{i, d_{i}} & \lambda_{i, d_{i}-1} & \cdots & \lambda_{i, 2} \\
0 & \lambda_{i, d_{i}} & \cdots & \lambda_{i, 3} \\
\vdots & \ddots & \ddots & \vdots \\
0 & \cdots & 0 & \lambda_{i, d_{i}}
\end{array}\right)\left(\begin{array}{c}
f_{d_{i}-1} \\
f_{d_{i}-2} \\
\vdots \\
f_{1}
\end{array}\right) \in \mathbb{C}\left(\begin{array}{c}
1 \\
0 \\
\vdots \\
0
\end{array}\right) .
$$

Since $\lambda_{i, d_{i}} \neq 0$, this means $f_{k}=0$ for all $k=1,2, \ldots, d_{i}-2$. Hence the stabilizer is given by

$$
\left\{g(z)=\operatorname{Id}_{V_{j}}+\sum_{k=1}^{d_{i}-1} g_{k} z^{k} \mid g_{k} \in \operatorname{Lie} K, k=1, \ldots, d_{i}-2, g_{d_{i}-1} \in \mathfrak{g l}\left(V_{j}\right)\right\} .
$$

The above implies that the orbit $\check{\mathcal{O}}$ is naturally isomorphic to

$$
\left(\mathfrak{g l}\left(V_{j}\right) / \operatorname{Lie} K\right)^{\oplus\left(d_{i}-2\right)} \simeq \operatorname{Hom}\left(V_{j} / V_{i}, V_{i}\right)^{\oplus\left(d_{i}-2\right)} \oplus \operatorname{Hom}\left(V_{i}, V_{j} / V_{i}\right)^{\oplus\left(d_{i}-2\right)} .
$$

Let us denote an element of the vector space on the right hand side by

$$
\left(a_{1}, \ldots, a_{d_{i}-2}, b_{1}, \ldots, b_{d_{i}-2}\right), \quad a_{k} \in \operatorname{Hom}\left(V_{j} / V_{i}, V_{i}\right), \quad b_{k} \in \operatorname{Hom}\left(V_{i}, V_{j} / V_{i}\right),
$$


and set $a(z):=\sum a_{k} z^{k}, b(z):=\sum_{k} b_{k} z^{k}$. Then the isomorphism is explicitly given by

$$
\left(a_{k}, b_{k}\right)_{k=1}^{d_{i}-2} \longmapsto g \cdot \Lambda^{0} \in \check{\mathcal{O}}, \quad g(z):=\left(\begin{array}{cc}
\operatorname{Id}_{V_{i}} & a(z) \\
b(z) & \operatorname{Id}_{V_{j} / V_{i}}
\end{array}\right) \in B_{d_{i}}\left(V_{j}\right)
$$

It is clearly $K$-equivariant.

Let us calculate the Kirillov-Kostant-Souriau symplectic form $\omega_{\check{\mathcal{O}}}$ on $\check{\mathcal{O}}$ in terms of the coordinates $(a, b)$. Let $\left(\delta_{l} a, \delta_{l} b\right), l=1,2$ be two tangent vectors at $(a, b)$. Then the corresponding tangent vectors at $g \cdot \Lambda^{0} \in \check{\mathcal{O}}$ are given by

$$
v_{l}=\left[\delta_{l} g \cdot g^{-1}, g \Lambda^{0} g^{-1}\right] \quad \bmod z^{-1} \mathfrak{g l}\left(V_{j}\right)[[z]] \quad \in \mathfrak{b}_{d_{i}}^{*}\left(V_{j}\right),
$$

where

$$
\delta_{l} g:=\left(\begin{array}{cc}
0 & \delta_{l} a(z) \\
\delta_{l} b(z) & 0
\end{array}\right) \in \mathfrak{b}_{d_{i}}\left(V_{j}\right), \quad l=1,2 .
$$

By the definition, we have

$$
\begin{aligned}
\omega_{\check{\mathcal{O}}}\left(v_{1}, v_{2}\right) & =\operatorname{tr} \operatorname{res}_{z=0}\left(g \Lambda^{0} g^{-1}\left[\delta_{1} g \cdot g^{-1}, \delta_{2} g \cdot g^{-1}\right]\right)=\operatorname{tr} \underset{z=0}{\operatorname{res}}\left(\Lambda^{0}\left[g^{-1} \delta_{1} g, g^{-1} \delta_{2} g\right]\right) \\
& =\operatorname{tr} \underset{z=0}{\operatorname{res}}\left(\left[\Lambda^{0}, g^{-1} \delta_{1} g\right] g^{-1} \delta_{2} g\right) .
\end{aligned}
$$

Using the obvious formula

$$
g(z)^{-1}=\left(\begin{array}{cc}
\operatorname{Id}_{V_{i}} & a(z) \\
b(z) & \operatorname{Id}_{V_{j} / V_{i}}
\end{array}\right)^{-1}=\left(\begin{array}{cc}
\left(\operatorname{Id}_{V_{i}}-a b\right)^{-1} & -a\left(\operatorname{Id}_{V_{j} / V_{i}}-b a\right)^{-1} \\
-b\left(\operatorname{Id}_{V_{i}}-a b\right)^{-1} & \left(\operatorname{Id}_{V_{j} / V_{i}}-b a\right)^{-1}
\end{array}\right)
$$

we have

$$
\begin{aligned}
g^{-1} \delta_{1} g & =\left(\begin{array}{cc}
\left(\operatorname{Id}_{V_{i}}-a b\right)^{-1} & -a\left(\operatorname{Id}_{V_{j} / V_{i}}-b a\right)^{-1} \\
-b\left(\operatorname{Id}_{V_{i}}-a b\right)^{-1} & \left(\operatorname{Id}_{V_{j} / V_{i}}-b a\right)^{-1}
\end{array}\right)\left(\begin{array}{cc}
0 & \delta_{1} a(z) \\
\delta_{1} b(z) & 0
\end{array}\right) \\
& =\left(\begin{array}{cc}
\left(\operatorname{Id}_{V_{i}}-a b\right)^{-1} \delta_{1} b & \left(\operatorname{Id}_{V_{i}}-a b\right)^{-1} \delta_{1} a \\
\left(\operatorname{Id}_{V_{j} / V_{i}}-b a\right)^{-1} \delta_{1} b & -b\left(\operatorname{Id}_{V_{i}}-a b\right)^{-1} \delta_{1} a
\end{array}\right),
\end{aligned}
$$

and hence

$$
\left[\Lambda^{0}, g^{-1} \delta_{1} g\right]=\left(\begin{array}{cc}
0 & \lambda_{i}^{0}\left(\operatorname{Id}_{V_{i}}-a b\right)^{-1} \delta_{1} a \\
-\lambda_{i}^{0}\left(\operatorname{Id}_{V_{j} / V_{i}}-b a\right)^{-1} \delta_{1} b & 0
\end{array}\right) .
$$

Substituting it into (A.3), we obtain

$$
\begin{aligned}
\omega_{\check{\mathcal{O}}}\left(v_{1}, v_{2}\right)= & \operatorname{tr} \operatorname{res}_{z=0}\left[\lambda_{i}^{0}\left(\operatorname{Id}_{V_{i}}-a b\right)^{-1} \delta_{1} a\left(\operatorname{Id}_{V_{j} / V_{i}}-b a\right)^{-1} \delta_{2} b\right] \\
& -\operatorname{tr} \underset{z=0}{\operatorname{res}}\left[\lambda_{i}^{0}\left(\operatorname{Id}_{V_{j} / V_{i}}-b a\right)^{-1} \delta_{1} b\left(\operatorname{Id}_{V_{i}}-a b\right)^{-1} \delta_{2} a\right],
\end{aligned}
$$

i.e.,

$$
\omega_{\check{\mathcal{O}}}=\operatorname{tr} \underset{z=0}{\operatorname{res}}\left[\lambda_{i}^{0}\left(\operatorname{Id}_{V_{i}}-a b\right)^{-1} \mathrm{~d} a \wedge\left(\operatorname{Id}_{V_{j} / V_{i}}-b a\right)^{-1} \mathrm{~d} b\right] .
$$

Now we set

$$
a_{k}^{\prime}:=\operatorname{res}_{z=0}\left[z^{k} \lambda_{i}^{0}\left(\operatorname{Id}_{V_{i}}-a b\right)^{-1} a\right], \quad b_{k}^{\prime}:=b_{k}, \quad k=1, \ldots, d_{i}-2 .
$$


Using $\left(\operatorname{Id}_{V_{i}}-a b\right)^{-1}=\sum_{l \geq 0}(a b)^{l}$, we see that $a_{k}^{\prime}$ is the sum of matrices

$$
\lambda_{i, m}\left(a_{p_{1}} b_{q_{1}}\right)\left(a_{p_{2}} b_{q_{2}}\right) \cdots\left(a_{p_{l}} b_{q_{l}}\right) a_{r}
$$

over all $l \geq 0$ and $m, p_{1}, \ldots, p_{l}, q_{1}, \ldots, q_{l}, r$ with $m=k+\sum p_{j}+\sum q_{j}+r+1$. Note that the indices for $a, b$ satisfy

$$
r \leq m-k-1 \leq d_{i}-k-1, \quad p_{j}, q_{j} \leq m-k-r-1<d_{i}-k-1,
$$

and $r=d_{i}-k-1$ only when $m=d_{i}$ and $l=0$. Thus we can write

$$
a_{k}^{\prime}=\lambda_{i, d_{i}} a_{d_{i}-k-1}+f_{k}\left(a_{1}, \ldots, a_{d_{i}-k-2}, b_{1}, \ldots, b_{d_{i}-k-2}\right)
$$

for some non-commutative polynomial $f_{k}$. Since $\lambda_{i, d_{i}} \neq 0$, the above implies that one can uniquely determine $\left(a_{k}, b_{k}\right)_{k=1}^{d_{i}-2}$ from $\left(a_{k}^{\prime}, b_{k}^{\prime}\right)_{k=1}^{d_{i}-2}$ in an algebraic way. Hence $\left(a_{k}, b_{k}\right)_{k=1}^{d_{i}-2} \mapsto$ $\left(a_{k}^{\prime}, b_{k}^{\prime}\right)_{k=1}^{d_{i}-2}$ is a biregular map. By the definition, it is clearly $K$-equivariant.

Let us calculate the 1 -form $\sum_{k=1}^{d_{i}-2} \operatorname{tr} \mathrm{d} a_{k}^{\prime} \wedge \mathrm{d} b_{k}^{\prime}$. First, we have

$$
\begin{aligned}
\mathrm{d}\left[\left(\operatorname{Id}_{V_{i}}-a b\right)^{-1} a\right]= & \mathrm{d}\left(\operatorname{Id}_{V_{i}}-a b\right)^{-1} \cdot a+\left(\operatorname{Id}_{V_{i}}-a b\right)^{-1} \mathrm{~d} a \\
= & \left(\operatorname{Id}_{V_{i}}-a b\right)^{-1} \mathrm{~d}(a b)\left(\operatorname{Id}_{V_{i}}-a b\right)^{-1} a+\left(\operatorname{Id}_{V_{i}}-a b\right)^{-1} \mathrm{~d} a \\
= & \left(\operatorname{Id}_{V_{i}}-a b\right)^{-1} \mathrm{~d} a\left[b\left(\operatorname{Id}_{V_{i}}-a b\right)^{-1} a+\operatorname{Id}_{V_{j} / V_{i}}\right] \\
& +\left(\operatorname{Id}_{V_{i}}-a b\right)^{-1} a \mathrm{~d} b\left(\operatorname{Id}_{V_{i}}-a b\right)^{-1} a .
\end{aligned}
$$

Note that the obvious equality $b\left(\operatorname{Id}_{V_{i}}-a b\right)=\left(\operatorname{Id}_{V_{j} / V_{i}}-b a\right) b$ implies

$$
b\left(\operatorname{Id}_{V_{i}}-a b\right)^{-1}=\left(\operatorname{Id}_{V_{j} / V_{i}}-b a\right)^{-1} b .
$$

Thus we have

$$
\begin{aligned}
\mathrm{d}\left[\left(\operatorname{Id}_{V_{i}}-a b\right)^{-1} a\right]= & \left(\operatorname{Id}_{V_{i}}-a b\right)^{-1} \mathrm{~d} a\left[\left(\operatorname{Id}_{V_{j} / V_{i}}-b a\right)^{-1} b a+\operatorname{Id}_{V_{j} / V_{i}}\right] \\
& +\left(\operatorname{Id}_{V_{i}}-a b\right)^{-1} a \mathrm{~d} b\left(\operatorname{Id}_{V_{i}}-a b\right)^{-1} a \\
= & \left(\operatorname{Id}_{V_{i}}-a b\right)^{-1} \mathrm{~d} a\left(\operatorname{Id}_{V_{j} / V_{i}}-b a\right)^{-1} \\
& +\left(\operatorname{Id}_{V_{i}}-a b\right)^{-1} a \mathrm{~d} b\left(\operatorname{Id}_{V_{i}}-a b\right)^{-1} a,
\end{aligned}
$$

and hence

$$
\begin{aligned}
\operatorname{tr}\left(\lambda_{i}^{0} \mathrm{~d}\left[\left(\operatorname{Id}_{V_{i}}-a b\right)^{-1} a\right] \wedge \mathrm{d} b\right)= & \operatorname{tr}\left[\lambda_{i}^{0}\left(\operatorname{Id}_{V_{i}}-a b\right)^{-1} \mathrm{~d} a \wedge\left(\operatorname{Id}_{V_{j} / V_{i}}-b a\right)^{-1} \mathrm{~d} b\right] \\
& +\operatorname{tr}\left[\lambda_{i}^{0}\left(\operatorname{Id}_{V_{i}}-a b\right)^{-1} a \mathrm{~d} b \wedge\left(\operatorname{Id}_{V_{i}}-a b\right)^{-1} a \mathrm{~d} b\right] \\
= & \operatorname{tr}\left[\lambda_{i}^{0}\left(\operatorname{Id}_{V_{i}}-a b\right)^{-1} \mathrm{~d} a \wedge\left(\operatorname{Id}_{V_{j} / V_{i}}-b a\right)^{-1} \mathrm{~d} b\right] .
\end{aligned}
$$

The above and (A.5) imply that the 1-form $\sum_{k=1}^{d_{i}-2} \operatorname{tr} \mathrm{d} a_{k}^{\prime} \wedge \mathrm{d} b_{k}^{\prime}$ coincides with $\omega_{\check{\mathcal{O}}}$; indeed,

$$
\begin{aligned}
\sum_{k=1}^{d_{i}-2} \operatorname{tr} \mathrm{d} a_{k}^{\prime} \wedge \mathrm{d} b_{k}^{\prime} & =\sum_{k=1}^{d_{i}-2} \operatorname{tr} \operatorname{res}_{z=0}\left(z^{k} \lambda_{i}^{0} \mathrm{~d}\left[\left(\operatorname{Id}_{V_{i}}-a b\right)^{-1} a\right] \wedge \mathrm{d} b_{k}\right) \\
& =\operatorname{res}_{z=0} \operatorname{tr}\left(\lambda_{i}^{0} \mathrm{~d}\left[\left(\operatorname{Id}_{V_{i}}-a b\right)^{-1} a\right] \wedge \mathrm{d} b\right) \\
& =\operatorname{res}_{z=0} \operatorname{tr}\left[\lambda_{i}^{0}\left(\operatorname{Id}_{V_{i}}-a b\right)^{-1} \mathrm{~d} a \wedge\left(\operatorname{Id}_{V_{j} / V_{i}}-b a\right)^{-1} \mathrm{~d} b\right]=\omega_{\check{\mathcal{O}}}
\end{aligned}
$$

Hence the map $\left(a_{k}^{\prime}, b_{k}^{\prime}\right)_{k=1}^{d_{i}-2} \mapsto g \cdot \Lambda^{0}$ is a $K$-equivariant symplectomorphism

$$
\operatorname{Hom}\left(V_{j} / V_{i}, V_{i}\right)^{\oplus\left(d_{i}-2\right)} \oplus \operatorname{Hom}\left(V_{i}, V_{j} / V_{i}\right)^{\oplus\left(d_{i}-2\right)} \simeq \check{\mathcal{O}} .
$$

Since this sends the origin to $\Lambda^{0}$, Lemma 5.2 follows. 


\section{A.2 Proof of Lemma 5.3}

First, we show the following lemma:

Lemma A.1. Let

$$
\check{\mathcal{O}} \rightarrow \operatorname{Lie} K, \quad B(z) \mapsto-\Gamma_{B} \in \operatorname{Lie} K
$$

be the $K$-moment map sending $\Lambda^{0}$ to zero. Then for any $B(z) \in \check{\mathcal{O}}$, there exists $g(z) \in B_{d_{i}}\left(V_{j}\right)$ such that

$$
g(z) B(z) g(z)^{-1}=\Lambda^{0}(z)+z^{-1} \Gamma_{B} \bmod \mathfrak{g l}\left(V_{j}\right)[[z]] .
$$

Proof. Let $B(z)=\sum_{k=2}^{d_{i}} B_{k} z^{-k} \in \check{\mathcal{O}}$, and let $a(z), b(z), g(z)$ be as in (A.2) such that $B=g \cdot \Lambda^{0}$. By the definition of the $B_{d_{i}}\left(V_{j}\right)$-action, we then have

$$
g(z)^{-1} B(z) g(z)=\Lambda^{0}(z)+z^{-1} \Gamma \bmod \mathfrak{g l}\left(V_{j}\right)[[z]]
$$

for some $\Gamma \in \mathfrak{g l}\left(V_{j}\right)$. According to the decomposition $V_{j}=V_{i} \oplus V_{j} / V_{i}$, we write it as

$$
\Gamma=\left(\begin{array}{ll}
\Gamma_{11} & \Gamma_{12} \\
\Gamma_{21} & \Gamma_{22}
\end{array}\right)
$$

and set

$$
\Gamma_{B}:=\left(\begin{array}{cc}
\Gamma_{11} & 0 \\
0 & \Gamma_{22}
\end{array}\right), \quad U:=\left(\begin{array}{cc}
0 & \lambda_{i, d_{i}}^{-1} \Gamma_{12} \\
-\lambda_{i, d_{i}}^{-1} \Gamma_{21} & 0
\end{array}\right), \quad u(z):=\operatorname{Id}_{V_{j}}+U z^{d_{i}-1} .
$$

Note that $\Gamma_{B} \in$ Lie $K$. Let $\Lambda_{d_{i}}$ be the top coefficient of $\Lambda^{0}(z)$. Then $U$ satisfies

$$
\left[\Lambda_{d_{i}}, U\right]=\left(\begin{array}{cc}
0 & \lambda_{i, d_{i}} \cdot \lambda_{i, d_{i}}^{-1} \Gamma_{12} \\
\left(-\lambda_{i, d_{i}}\right) \cdot-\lambda_{i, d_{i}}^{-1} \Gamma_{21} & 0
\end{array}\right)=\Gamma-\Gamma_{B}
$$

and hence

$$
\begin{aligned}
u(z) g(z)^{-1} B(z) g(z) u(z) & =u(z)\left(\Lambda^{0}(z)+z^{-1} \Gamma\right) u(z)^{-1} & & \bmod \mathfrak{g l}\left(V_{j}\right)[[z]] \\
& =\Lambda^{0}(z)+z^{-1} \Gamma+z^{-1}\left[U, \Lambda_{d_{i}}\right] & & \bmod \mathfrak{g l}\left(V_{j}\right)[[z]] \\
& =\Lambda^{0}(z)+z^{-1} \Gamma_{B} & & \bmod \mathfrak{g l}\left(V_{j}\right)[[z]]
\end{aligned}
$$

Now we explicitly describe $\Gamma_{B}$ in terms of the coordinates $\left(a_{k}^{\prime}, b_{k}^{\prime}\right)_{k=1}^{d_{i}-2}$, which shows that $B \mapsto-\Gamma_{B}$ is a $K$-moment map. Note that the constant term of $g(z)$ is the identity, and hence it acts trivially on $z^{-1} \mathfrak{g l}\left(V_{j}\right)[[z]] / \mathfrak{g l}\left(V_{j}\right)[[z]]$ by conjugation. Therefore (A.7) implies

$$
\begin{aligned}
B(z) & =g(z)\left(\Lambda^{0}(z)+z^{-1} \Gamma\right) g(z)^{-1} & & \bmod \mathfrak{g l}\left(V_{j}\right)[[z]] \\
& =g(z) \Lambda^{0}(z) g(z)^{-1}+z^{-1} \Gamma & & \bmod \mathfrak{g l}\left(V_{j}\right)[[z]] .
\end{aligned}
$$

Substituting (A.4) into the above equality, we have

$$
B(z)=\left(\begin{array}{cc}
\lambda_{i}^{0}\left(\operatorname{Id}_{V_{i}}-a b\right)^{-1} & -\lambda_{i}^{0} a\left(\operatorname{Id}_{V_{j} / V_{i}}-b a\right)^{-1} \\
b \lambda_{i}^{0}\left(\operatorname{Id}_{V_{i}}-a b\right)^{-1} & -b \lambda_{i}^{0} a\left(\operatorname{Id}_{V_{j} / V_{i}}-b a\right)^{-1}
\end{array}\right)+\frac{\Gamma}{z} \quad \bmod \mathfrak{g l}\left(V_{j}\right)[[z]] .
$$


Note that $B(z)$ and $\lambda_{i}^{0}(z)$ have no residue parts. Looking at the block diagonal part of the above and taking the residue, we thus obtain

$$
\begin{aligned}
\Gamma_{11} & =-\underset{z=0}{\operatorname{res}}\left[\lambda_{i}^{0}\left(\operatorname{Id}_{V_{i}}-a b\right)^{-1}\right]=-\sum_{l=0}^{\infty} \underset{z=0}{\operatorname{res}}\left[\lambda_{i}^{0}(a b)^{l}\right]=-\sum_{l=1}^{\infty} \underset{z=0}{\operatorname{res}}\left[\lambda_{i}^{0}(a b)^{l}\right] \\
& =-\underset{z=0}{\operatorname{res}}\left[\lambda_{i}^{0}\left(\operatorname{Id}_{V_{i}}-a b\right)^{-1} a b\right]=-\sum_{k} \operatorname{res}_{z=0}\left[z^{k} \lambda_{i}^{0}\left(\operatorname{Id}_{V_{i}}-a b\right)^{-1} a\right] b_{k}=-\sum_{k} a_{k}^{\prime} b_{k}^{\prime},
\end{aligned}
$$

and similarly,

$$
\Gamma_{22}=\underset{z=0}{\operatorname{res}}\left[b \lambda_{i}^{0} a\left(\operatorname{Id}_{V_{j} / V_{i}}-b a\right)^{-1}\right]=\sum_{k} b_{k}^{\prime} a_{k}^{\prime} .
$$

Hence

$$
\Gamma_{B}=-\sum_{k}\left(\begin{array}{cc}
a_{k}^{\prime} b_{k}^{\prime} & 0 \\
0 & -b_{k}^{\prime} a_{k}^{\prime}
\end{array}\right)
$$

which gives the minus of the $K$-moment map vanishing at $a_{k}^{\prime}, b_{k}^{\prime}=0$.

Remark A.2. The matrix $\Gamma$ in (A.7) is characterized by $\Gamma=\underset{z=0}{\operatorname{res}} g(z)^{-1} B(z) g(z)$, so that it depends algebraically on $a_{k}, b_{k}$. Hence $u(z) g(z)^{-1}$ also depends algebraically on $a_{k}, b_{k}$. This means that one can choose $g(z)$ in the assertion of Lemma A.1 so that it depends algebraically on $B \in \check{\mathcal{O}}$.

Remark A.3. In the above proof, let us write

$$
B(z)=\left(\begin{array}{ll}
B_{11}(z) & B_{12}(z) \\
B_{21}(z) & B_{22}(z)
\end{array}\right)
$$

Then (A.8) implies

$$
\begin{aligned}
& B_{11}(z)=\lambda_{i}^{0}\left(\operatorname{Id}_{V_{i}}-a b\right)^{-1} \quad \bmod z^{-1} \mathfrak{g l}\left(V_{i}\right)[[z]], \\
& B_{12}(z)=-\lambda_{i}^{0} a\left(\operatorname{Id}_{V_{j} / V_{i}}-b a\right)^{-1} \quad \bmod \operatorname{Hom}\left(V_{j} / V_{i}, V_{i}\right) \otimes z^{-1} \mathbb{C}[[z]], \\
& B_{21}(z)=b \lambda_{i}^{0}\left(\operatorname{Id}_{V_{i}}-a b\right)^{-1} \quad \bmod \operatorname{Hom}\left(V_{i}, V_{j} / V_{i}\right) \otimes z^{-1} \mathbb{C}[[z]], \\
& B_{22}(z)=-b \lambda_{i}^{0} a\left(\operatorname{Id}_{V_{j} / V_{i}}-b a\right)^{-1} \quad \bmod z^{-1} \mathfrak{g l}\left(V_{j} / V_{i}\right)[[z]] \text {. }
\end{aligned}
$$

Note that $\lambda_{i}^{0}\left(\operatorname{Id}_{V_{i}}-a b\right)^{-1} a$ has pole order $d_{i}-1$ and

$$
\lambda_{i}^{0}\left(\operatorname{Id}_{V_{i}}-a b\right)^{-1} a=\sum_{k=1}^{d_{i}-2} a_{k}^{\prime} z^{-k-1} \bmod \operatorname{Hom}\left(V_{j} / V_{i}, V_{i}\right) \otimes z^{-1} \mathbb{C}[[z]] .
$$

Set $a^{\prime}(z):=\sum_{k=1}^{d_{i}-2} a_{k}^{\prime} z^{-k-1}$. Using the obvious formulas $a\left(\operatorname{Id}_{V_{j} / V_{i}}-b a\right)^{-1}=\left(\operatorname{Id}_{V_{i}}-a b\right)^{-1} a$ and $\left(\operatorname{Id}_{V_{i}}-a b\right)^{-1}=\operatorname{Id}_{V_{i}}+\left(\operatorname{Id}_{V_{i}}-a b\right)^{-1} a b$, we can then rewrite the above four equalities as

$$
\begin{array}{llrl}
B_{11}(z) & =\lambda_{i}^{0} \operatorname{Id}_{V_{i}}+a^{\prime} b^{\prime} & & \bmod z^{-1} \mathfrak{g r}\left(V_{i}\right)[[z]], \\
B_{12}(z) & =-a^{\prime}, & & \\
B_{21}(z) & =\lambda_{i}^{0} b^{\prime}+b^{\prime} a^{\prime} b^{\prime} & & \bmod \operatorname{Hom}\left(V_{i}, V_{j} / V_{i}\right) \otimes z^{-1} \mathbb{C}[[z]], \\
B_{22}(z) & =-b^{\prime} a^{\prime} & & \bmod z^{-1} \mathfrak{g r}\left(V_{j} / V_{i}\right)[[z]],
\end{array}
$$


which give the explicit description of $B$ in terms of the coordinates $\left(a_{k}^{\prime}, b_{k}^{\prime}\right)$. Conversely, we can describe $\left(a^{\prime}, b^{\prime}\right)$ in terms of $B$ using the above. Indeed, (A.10) determines $a^{\prime}$, and (A.9) and (A.11) imply

$$
B_{21}(z)=b^{\prime}(z) B_{11}(z) \bmod \operatorname{Hom}\left(V_{i}, V_{j} / V_{i}\right) \otimes z^{-1} \mathbb{C}[[z]] .
$$

Writing $B_{i j}=\sum_{k} B_{i j, k} z^{-k}$, we then have

$$
\left(\begin{array}{lll}
B_{21, d_{i}-1} & \cdots & B_{21,2}
\end{array}\right)=\left(\begin{array}{lll}
b_{1}^{\prime} & \cdots & b_{d_{i}-2}^{\prime}
\end{array}\right)\left(\begin{array}{cccc}
B_{11, d_{i}} & B_{11, d_{i}-1} & \cdots & B_{11,3} \\
0 & B_{11, d_{i}} & \cdots & B_{11,4} \\
\vdots & \ddots & \ddots & \vdots \\
0 & \cdots & 0 & B_{11, d_{i}}
\end{array}\right) .
$$

Note that (A.9) also shows $B_{11, d_{i}}=\lambda_{i, d_{i}} \operatorname{Id}_{V_{i}}$. Hence the block matrix on the far right is invertible, and therefore we can express $b_{k}^{\prime}$ as

$$
b_{k}^{\prime}=\sum_{l=2}^{d_{i}-1} B_{21, l} F_{l k}\left(B_{11,3}, \ldots, B_{11, d_{i}-1}\right)
$$

with some non-commutative polynomial $F_{l k}$.

Proof of Lemma 5.3. We give a proof of Lemma 5.3. Let $\varphi: \check{\mathcal{O}} \times M \rightarrow \mathfrak{g}_{d_{i}}^{*}\left(V_{j}\right) \times M$ be the map defined in its statement;

$$
\varphi(B(z), x)=(A(z), x), \quad A(z):=B(z)-\frac{\mu_{M}(x)+\zeta \operatorname{Id}_{V_{j}}}{z},
$$

which is clearly equivariant under the conjugation by $K$. Now suppose that $(B(z), x) \in \check{\mathcal{O}} \times M$ satisfies the moment map condition

$$
\check{\mu}(B, x):=-\Gamma_{B}+\mu_{M}(x)=-\underset{z=0}{\operatorname{res}} \Lambda(z)-\zeta \operatorname{Id}_{V_{j}} .
$$

By Lemma A.1, there exists $g(z) \in B_{d_{i}}\left(V_{j}\right)$ such that

$$
B(z)=g(z)\left(\Lambda^{0}(z)+z^{-1} \Gamma_{B}\right) g(z)^{-1} \bmod \mathfrak{g l}\left(V_{j}\right)[[z]] .
$$

Noting that the constant term $g(0)$ of $g(z)$ is the identity, we obtain

$$
\begin{aligned}
A(z) & =g(z)\left(\Lambda^{0}(z)+\frac{\Gamma_{B}}{z}\right) g(z)^{-1}-\frac{\mu_{M}(x)+\zeta \operatorname{Id}_{V_{j}}}{z} & & \bmod \mathfrak{g r}\left(V_{j}\right)[[z]] \\
& =g(z) \Lambda^{0}(z) g(z)^{-1}+\frac{\Gamma_{B}-\mu_{M}(x)-\zeta \operatorname{Id}_{V_{j}}}{z} & & \bmod \mathfrak{g r}\left(V_{j}\right)[[z]] \\
& =g(z) \Lambda^{0}(z) g(z)^{-1}+\frac{\operatorname{res} \Lambda}{z} & & \bmod \mathfrak{g l}\left(V_{j}\right)[[z]] \\
& =g(z)\left(\Lambda^{0}(z)+\frac{\underset{z=0}{\operatorname{res}} \Lambda}{z}\right) g(z)^{-1} & & \bmod \mathfrak{g r}\left(V_{j}\right)[[z]] \\
& =g(z) \Lambda(z) g(z)^{-1} & & \bmod \mathfrak{g r}\left(V_{j}\right)[[z]],
\end{aligned}
$$

which implies $A(z) \in \mathcal{O}$. Since $B(z)$ has no residue, we have $\underset{z=0}{\operatorname{res}} A(z)=-\mu_{M}(x)-\zeta \operatorname{Id}_{V_{j}}$, in other words, the value of the $\mathrm{GL}\left(V_{j}\right)$-moment map

$$
\mu: \mathcal{O} \times M \rightarrow \mathfrak{g l}\left(V_{j}\right), \quad(A, x) \mapsto \underset{z=0}{\operatorname{res}} A(z)+\mu_{M}(x)
$$


at $\varphi(B, x)$ is $-\zeta \operatorname{Id}_{V_{j}}$. Hence $\varphi$ induces a map between the symplectic quotients

$$
\bar{\varphi}: \check{\mu}^{-1}\left(-\underset{z=0}{\operatorname{res}} \Lambda-\zeta \operatorname{Id}_{V_{j}}\right) / K \longrightarrow \mu^{-1}\left(-\zeta \operatorname{Id}_{V_{j}}\right) / \mathrm{GL}\left(V_{j}\right) .
$$

We show that the above map is bijective. Suppose that $(B, x),\left(B^{\prime}, x^{\prime}\right) \in \check{\mu}^{-1}\left(-\operatorname{res}_{z=0} \Lambda-\zeta \operatorname{Id}_{V_{j}}\right)$ and $g \in \mathrm{GL}\left(V_{j}\right)$ satisfy $g \cdot \varphi(B, x)=\varphi\left(B^{\prime}, x^{\prime}\right)$. Then $g \cdot x=x^{\prime}$ and

$$
\begin{aligned}
g\left(B(z)-\frac{\mu_{M}(x)+\zeta \operatorname{Id}_{V_{j}}}{z}\right) g^{-1} & =B^{\prime}(z)-\frac{\mu_{M}\left(x^{\prime}\right)+\zeta \operatorname{Id}_{V_{j}}}{z}=B^{\prime}(z)-\frac{g \mu_{M}(x) g^{-1}+\zeta \operatorname{Id}_{V_{j}}}{z} \\
& =B^{\prime}(z)-g \frac{\mu_{M}(x)+\zeta \operatorname{Id}_{V_{j}}}{z} g^{-1} .
\end{aligned}
$$

Hence $g B(z) g^{-1}=B^{\prime}(z)$. Since $B, B^{\prime} \in \check{\mathcal{O}}$, their top coefficients are $\Lambda_{d_{i}}=\lambda_{i, d_{i}} \operatorname{Id}_{V_{i}} \oplus$ $0 \mathrm{Id}_{V_{j} / V_{i}}$, whose centralizer is $\mathrm{GL}\left(V_{i}\right) \times \mathrm{GL}\left(V_{j} / V_{i}\right)=K$. By comparing the top coefficients of $g B(z) g^{-1}, B^{\prime}(z)$, we thus obtain $g \in K$, and hence $(B, x)$ and $\left(B^{\prime}, x^{\prime}\right)$ lie in the same $K$-orbit. To prove the surjectivity, suppose that $(A, x) \in \mu^{-1}\left(-\zeta \mathrm{Id}_{V_{j}}\right)$ is given. By using the $\mathrm{GL}\left(V_{j}\right)$ action if necessary, we may assume that $A=g \cdot \Lambda$ for some $g(z) \in B_{d_{i}}\left(V_{j}\right)$ (if $A=g \cdot \Lambda$ for $g(z) \in G_{d_{i}}\left(V_{j}\right)$, we replace $(A, x)$ with $\left.g(0)^{-1} \cdot(A, x)\right)$. Let $B(z) \in \mathfrak{b}_{d_{i}}^{*}\left(V_{j}\right)$ be the residue-free part of $A(z)$. Taking modulo $z^{-1} \mathfrak{g l}\left(V_{j}\right)[[z]]$ of $A=g \cdot \Lambda$, we then have $B=g \cdot \Lambda^{0} \in \check{\mathcal{O}}$. Furthermore, the moment map condition for $(A, x)$ implies

$$
A(z)=B(z)+\frac{\operatorname{res}_{z=0} A}{z}=B(z)-\frac{\mu_{M}(x)+\zeta \operatorname{Id}_{V_{j}}}{z} .
$$

Hence $(B, x)=\varphi(A, x)$. This shows that $\bar{\varphi}$ is surjective.

We have proved that $\bar{\varphi}$ is bijective. Furthermore, by letting $(B, x)=\left(B^{\prime}, x^{\prime}\right)$ in the proof of the injectivity, we see that the stabilizer of $\varphi(B, x)$ with respect to the $\operatorname{GL}\left(V_{j}\right)$-action is contained in that of $(B, x)$ with respect to the $K$-action. The converse is clear from the $K$-equivariance of $\varphi$, and hence the two stabilizers coincide. In particular, free $K$-orbits correspond to free $\mathrm{GL}\left(V_{j}\right)$-orbits via $\varphi$, which is the second assertion of Lemma 5.3.

Finally, we show that $\bar{\varphi}$ preserves the symplectic structure at points representing free orbits. Let $(B, x)$ be a point in the level set $\check{\mu}^{-1}\left(-\underset{z=0}{\operatorname{res}} \Lambda-\zeta \operatorname{Id}_{V_{j}}\right)$ whose stabilizer is trivial (so the level set is smooth at $(B, x))$, and let $(A, x)=\varphi(B, x)$. We take $g(z) \in B_{d_{i}}\left(V_{j}\right)$ satisfying (A.12) so that it depends smoothly on $B$, which is possible as mentioned in Remark A.2. Then the argument just after (A.12) shows $A=g \cdot \Lambda$, and furthermore, the smoothness of $g$ implies that for any tangent vector $(\delta B, v)$ at $(B, x)$, there exists $\delta g \in \mathfrak{b}_{d_{i}}\left(V_{j}\right)$ such that

$$
\begin{aligned}
\delta B & =\left[\delta g \cdot g^{-1}, B\right] & & \bmod z^{-1} \mathfrak{g l}\left(V_{j}\right)[[z]], \\
\delta A & =\left[\delta g \cdot g^{-1}, A\right] & & \bmod \mathfrak{g l}\left(V_{j}\right)[[z]],
\end{aligned}
$$

where $(\delta A, v)=\varphi_{*}(\delta B, v)$ is the corresponding tangent vector at $(A, x)$. Now let $\left(\delta_{i} B, v_{i}\right)$, $i=1,2$ be two tangent vectors at $(B, x)$ and $\delta_{i} A, \delta_{i} g$ as above. Let $\omega_{\mathcal{O}}$ (resp. $\omega_{M}$ ) be the symplectic form on $\mathcal{O}$ (resp. $M$ ). By the definition, we have

$$
\omega_{\mathcal{O}}\left(\delta_{1} A, \delta_{2} A\right)=\operatorname{tr} \underset{z=0}{\operatorname{res}}\left(A\left[\delta_{1} g \cdot g^{-1}, \delta_{2} g \cdot g^{-1}\right]\right) .
$$

Since $\delta_{i} g$ has no constant term, we have $\left[\delta_{1} g \cdot g^{-1}, \delta_{2} g \cdot g^{-1}\right] \in z^{2} \mathfrak{g l}\left(V_{j}\right)[[z]]$, which implies

$$
\operatorname{tr} \operatorname{res}_{z=0}\left(A\left[\delta_{1} g \cdot g^{-1}, \delta_{2} g \cdot g^{-1}\right]\right)=\operatorname{tr} \operatorname{res}_{z=0}\left(B\left[\delta_{1} g \cdot g^{-1}, \delta_{2} g \cdot g^{-1}\right]\right)=\omega_{\check{\mathcal{O}}}\left(\delta_{1} B, \delta_{2} B\right),
$$

and hence

$$
\omega_{\mathcal{O}}\left(\delta_{1} A, \delta_{2} A\right)+\omega_{M}\left(v_{1}, v_{2}\right)=\omega_{\check{\mathcal{O}}}\left(\delta_{1} B, \delta_{2} B\right)+\omega_{M}\left(v_{1}, v_{2}\right) .
$$

This shows the assertion. 


\section{Acknowledgements}

I am grateful to Philip Boalch for stimulating conversations, and to Professor Hiraku Nakajima for valuable comments. This work was supported by the grants ANR-08-BLAN-0317-01 of the Agence nationale de la recherche and JSPS Grant-in-Aid for Scientific Research (S-19104002).

\section{References}

[1] Arinkin D., Fourier transform and middle convolution for irregular D-modules, arXiv:0808.0699.

[2] Boalch P., Symplectic manifolds and isomonodromic deformations, Adv. Math. 163 (2001), 137-205.

[3] Boalch P., From Klein to Painlevé via Fourier, Laplace and Jimbo, Proc. London Math. Soc. (3) 90 (2005), 167-208, math.AG/0308221.

[4] Boalch P., Irregular connections and Kac-Moody root systems, arXiv:0806.1050.

[5] Boalch P., Quivers and difference Painlevé equations, in Proceedings of Conference "Groups and Symmetries" (April 27-29, 2007), Editors J. Harnad and P. Winternitz, CRM Proc. Lecture Notes, Vol. 47, Amer. Math. Soc., Providence, RI, 2009, 25-51, arXiv:0706.2634.

[6] Crawley-Boevey W., Geometry of the moment map for representations of quivers, Compositio Math. 126 (2001), 257-293.

[7] Crawley-Boevey W., On matrices in prescribed conjugacy classes with no common invariant subspace and sum zero, Duke Math. J. 118 (2003), 339-352, math.RA/0103101.

[8] Crawley-Boevey W., Holland M.P., Noncommutative deformations of Kleinian singularities, Duke Math. J. 92 (1998), 605-635.

[9] Etingof P., Golberg O., Hensel S., Liu T., Schwendner A., Udovina E., Vaintrob D., Introduction to representation theory, arXiv:0901.0827.

[10] Harnad J., Dual isomonodromic deformations and moment maps to loop algebras, Comm. Math. Phys. 166 (1994), 337-365, hep-th/9301076.

[11] Hartshorne R., Algebraic geometry, Graduate Texts in Mathematics, Vol. 52, Springer-Verlag, New York Heidelberg, 1977.

[12] Jimbo M., Miwa T., Monodromy preserving deformation of linear ordinary differential equations with rational coefficients. II, Phys. D 2 (1981), 407-448.

[13] Kac V.G., Infinite-dimensional Lie algebras, 3rd ed., Cambridge University Press, Cambridge, 1990.

[14] Katz N.M., Rigid local systems, Annals of Mathematics Studies, Vol. 139, Princeton University Press, Princeton, NJ, 1996.

[15] Kawakami H., Generalized Okubo systems and the middle convolution, Int. Math. Res. Not. 2010 (2010), 3394-3421.

[16] King A.D., Moduli of representations of finite-dimensional algebras, Quart. J. Math. Oxford Ser. (2) 45 (1994), 515-530.

[17] Kronheimer P.B., The construction of ALE spaces as hyper-Kähler quotients, J. Differential Geom. 29 (1989), 665-683.

[18] Lusztig G., On quiver varieties, Adv. Math. 136 (1998), 141-182.

[19] Lusztig G., Quiver varieties and Weyl group actions, Ann. Inst. Fourier (Grenoble) 50 (2000), 461-489.

[20] Mumford D., Fogarty J., Kirwan F., Geometric invariant theory, 3rd ed., Ergebnisse der Mathematik und ihrer Grenzgebiete (2), Vol. 34, Springer-Verlag, Berlin, 1994.

[21] Nakajima H., Instantons on ALE spaces, quiver varieties, and Kac-Moody algebras, Duke Math. J. 76 (1994), 365-416.

[22] Nakajima H., Reflection functors for quiver varieties and Weyl group actions, Math. Ann. 327 (2003), 671-721.

[23] Okamoto K., Studies on the Painlevé equations. III. Second and fourth Painlevé equations, $P_{\mathrm{II}}$ and $P_{\mathrm{IV}}$, Math. Ann. 275 (1986), 221-255.

[24] Okamoto K., Studies on the Painlevé equations. I. Sixth Painlevé equation P PI, Ann. Mat. Pura Appl. (4) 146 (1987), 337-381. 
[25] Okamoto K., Studies on the Painlevé equations. II. Fifth Painlevé equation P $\mathrm{V}$, Japan. J. Math. (N.S.) 13 (1987), 47-76.

[26] Okamoto K., Studies on the Painlevé equations. IV. Third Painlevé equation PIII, Funkcial. Ekvac. 30 (1987), 305-332.

[27] van der Put M., Saito M.-H., Moduli spaces for linear differential equations and the Painlevé equations, Ann. Inst. Fourier (Grenoble) 59 (2009), 2611-2667, arXiv:0902.1702.

[28] Rump W., Doubling a path algebra, or: how to extend indecomposable modules to simple modules, in Proceedings of Workshop "Representation Theory of Groups, Algebras, and Orders" (Constanţa, 1995), Editors K.W. Roggenkamp and M. Ştefănescu, An. Ştiinţ. Univ. Ovidius Constanţa Ser. Mat. 4 (1996), 174-185.

[29] Sasano Y., Symmetry in the Painlevé systems and their extensions to four-dimensional systems, Funkcial. Ekvac. 51 (2008), 351-369, arXiv:0704.2393.

[30] Woodhouse N.M.J., Duality for the general isomonodromy problem, J. Geom. Phys. 57 (2007), 1147-1170, nlin.SI/0601003.

[31] Yamakawa D., Middle convolution and Harnad duality, Math. Ann., to appear, arXiv:0911.3863. 